\title{
WestVirginiaUniversity
}

THE RESEARCH REPOSITORY @ WVU

Graduate Theses, Dissertations, and Problem Reports

2019

\section{A Biomimetic Approach to Controlling Restorative Robotics}

Matthew T. Boots

mboots@mix.wvu.edu

Follow this and additional works at: https://researchrepository.wvu.edu/etd

Part of the Acoustics, Dynamics, and Controls Commons, Biomechanical Engineering Commons, Biomedical Devices and Instrumentation Commons, Neurosciences Commons, and the Orthotics and Prosthetics Commons

\section{Recommended Citation}

Boots, Matthew T., "A Biomimetic Approach to Controlling Restorative Robotics" (2019). Graduate Theses, Dissertations, and Problem Reports. 7384.

https://researchrepository.wvu.edu/etd/7384

This Dissertation is protected by copyright and/or related rights. It has been brought to you by the The Research Repository @ WVU with permission from the rights-holder(s). You are free to use this Dissertation in any way that is permitted by the copyright and related rights legislation that applies to your use. For other uses you must obtain permission from the rights-holder(s) directly, unless additional rights are indicated by a Creative Commons license in the record and/ or on the work itself. This Dissertation has been accepted for inclusion in WVU Graduate Theses, Dissertations, and Problem Reports collection by an authorized administrator of The Research Repository @ WVU.

For more information, please contact researchrepository@mail.wvu.edu. 


\title{
A Biomimetic Approach to Controlling Restorative Robotics
}

\author{
Matthew Thomas Boots \\ Dissertation submitted to the Mechanical and Aerospace Department \\ at West Virginia University \\ In partial fulfillment of the requirements for the degree of \\ Doctor of Philosophy in \\ Mechanical Engineering \\ Sergiy Yakovenko, PhD, Chair \\ Robert Gaunt, PhD \\ Yu Gu, PhD \\ Sam Mukdadi, PhD \\ Victor Mucino, PhD
}

Mechanical Engineering Graduate Program

Morgantown, WV

2019

Keywords: control system, motor control, computational neuroscience, myoelectric prostheses, musculoskeletal modeling, self-paced treadmill, split-belt treadmill, virtual reality

Copyright 2019 Matthew Thomas Boots 


\title{
Abstract \\ A Biomimetic Approach to Controlling Restorative Robotics
}

\author{
Matthew Thomas Boots
}

Movement is the only way a person can interact with the world around them. When trauma to the neuromuscular systems disrupts the control of movement, quality of life suffers. To restore limb functionality, active robotic interventions and/or rehabilitation are required. Unfortunately, the primary obstacle in a person's recovery is the limited robustness of the human-machine interfaces. Current systems rely on control approaches that rely on the person to learn how the system works instead of the system being more intuitive and working with the person naturally. My research goal is to design intuitive control mechanisms based on biological processes termed the biomimetic approach. I have applied this control scheme to problems with restorative robotics focused on the upper and lower limb control.

Operating an advanced active prosthetic hand is a two-pronged problem of actuating a highdimensional mechanism and controlling it with an intuitive interface. Our approach attempts to solve these problems by going from muscle activity, electromyography (EMG), to limb kinematics calculated through dynamic simulation of a musculoskeletal model. This control is more intuitive to the user because they attempt to move their hand naturally, and the prosthetic hand performs that movement. The key to this approach was validating simulated muscle paths using both experimental measurements and anatomical constraints where data is missing. After the validation, simulated muscle paths and forces are used to decipher the intended movement. After we have calculated the intended movement, we can move a prosthetic hand to match. This approach required minimal training to give an amputee the ability to control prosthetic hand movements, such as grasping. A more intuitive controller has the potential to improve how people interact and use their prosthetic hands.

Similarly, the rehabilitation of the locomotor system in people with damaged motor pathways or missing limbs require appropriate interventions. The problem of decoding human motor intent in a treadmill walking task can be solved with a biomimetic approach. Estimated limb speed is essential for this approach according to the theoretical input-output computation performed by spinal central pattern generators (CPGs), which represents neural circuitry responsible for autonomous control of stepping. The system used the locomotor phases, swing and stance, to estimate leg speeds and enable self-paced walking as well as steering in virtual reality with congruent visual flow. The unique advantage of this system over the previous state-of-art is the independent leg speed control, which is required for multidirectional movement in VR. This system has the potential to contribute to VR gait rehab techniques.

Creating biologically-inspired controllers has the potential to improve restorative robotics and allow people a better opportunity to recover lost functionality post-injury. 


\section{Acknowledgments}

I would like to thank many people that have helped me throughout my journey in graduate school.

First, I would like to thank my advisor, Dr. Sergiy Yakavenko, for all the help and advice he has provided me. I have learned several vital skills from working with him, including, but not limited to, conducting scientific research, running a laboratory, and critical thinking. I have grown exponentially as a researcher and a scientist from working with him over these last few years.

I would like to thank all my committee members Dr. Victor Mucino, Dr. Yu Gu, Dr. Osama Mukdadi, and Dr. Robert Gaunt, for their help and exclusive input on my thesis work.

I would also like to thank my wife, Theresa, and family for supporting me from start to finish of my graduate work.

Thank you to all my lab mates for the help they have provided me. Specifically, I would like to acknowledge Russell Hardesty, Trevor Moon, Dr. Anton Sobinov, Brad Pollard, Lisa Kogan, Dr. Erienne Olesh, Ariel Thomas, and Olivia Santee.

Thank you also to the RNEL laboratory at Pittsburgh University. Specifically, I would like to acknowledge Dr. Robert Gaunt, Dr. Lee Fisher, Dr. Jennifer Collinger, Dr. Douglas Weber, Dr. Michael Boninger, Dr. Misagh Mansouri, Carl Beringer, Tyler Simpson, and David Weir.

Lastly, I would like to thank all my friends for their continued support. 


\section{Table of Contents}

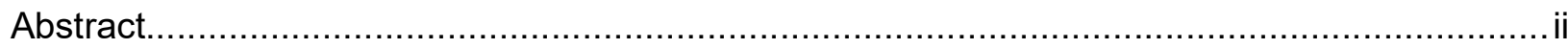

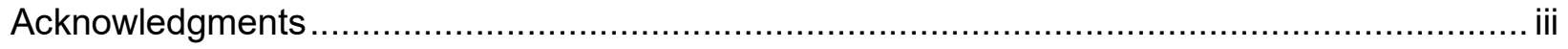

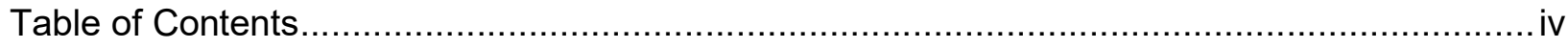

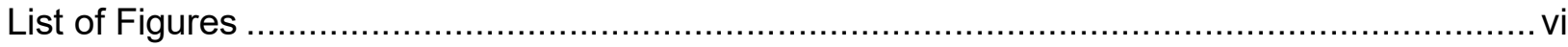

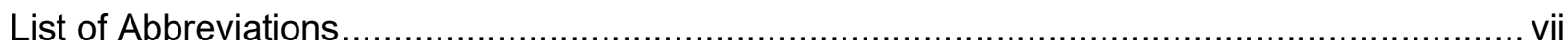

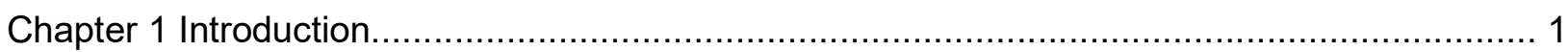

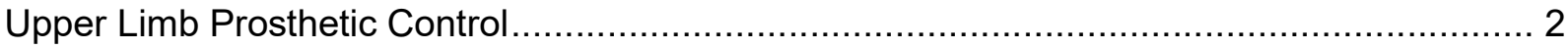

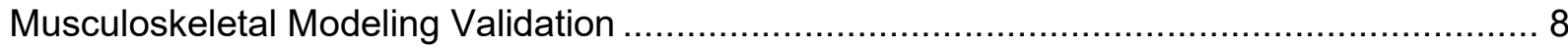

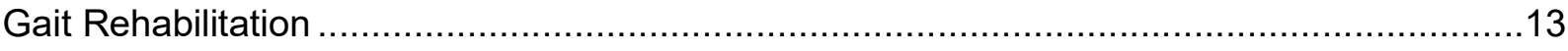

Chapter 2 Validating a Realistic Musculoskeletal Model for the Arm and Hand .....................17

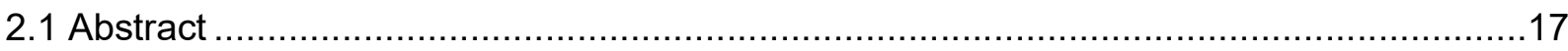

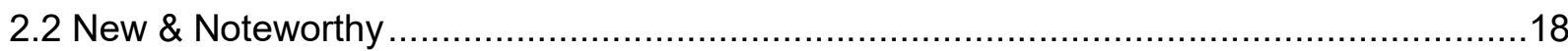

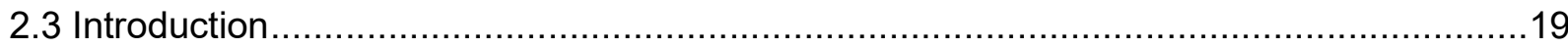

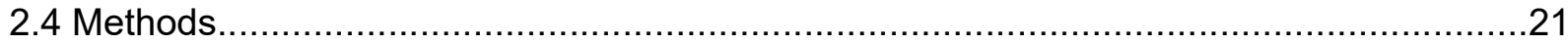

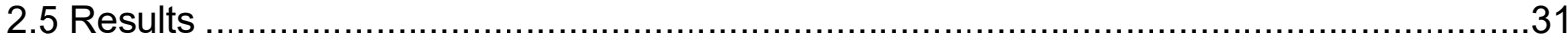

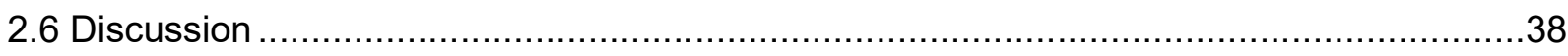

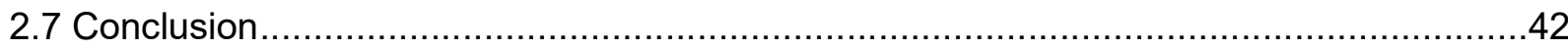

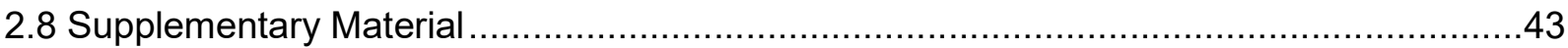

Chapter 3 Prosthetic Controller Approach Using A Refined Musculoskeletal Model ................44

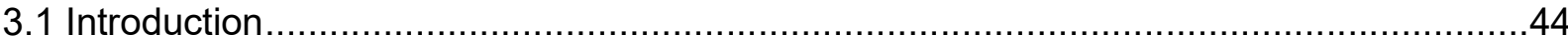

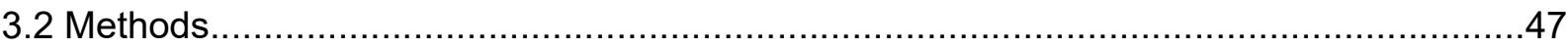

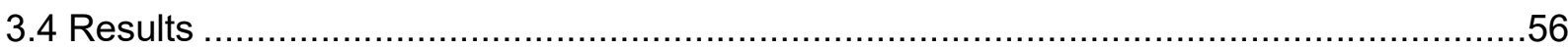

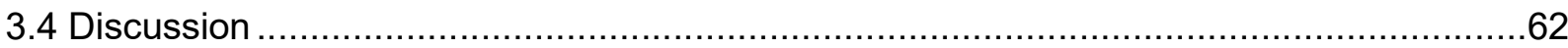

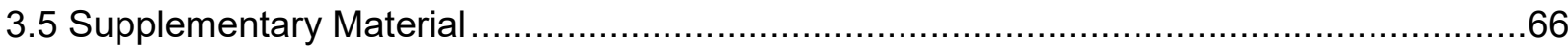

Chapter 4 Self-paced Omnidirectional Locomotion in Virtual Reality Using a Human-in-the-Loop

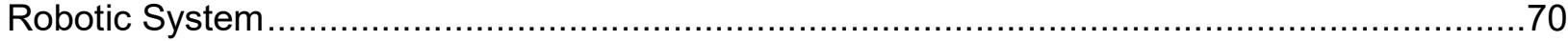

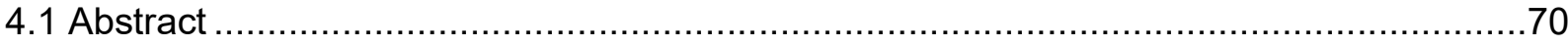

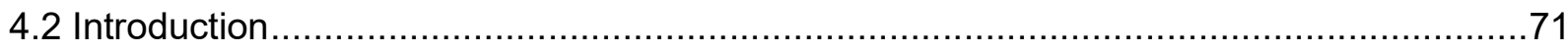

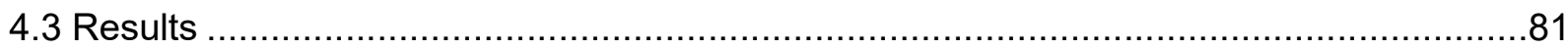

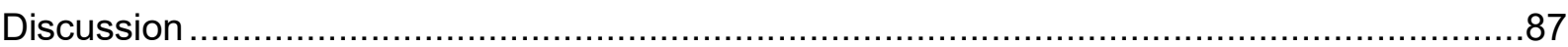

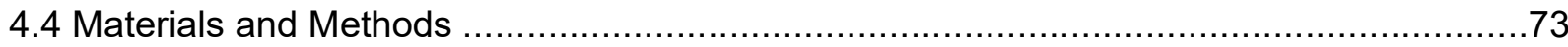

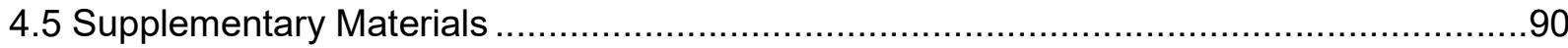

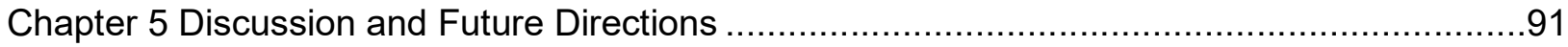

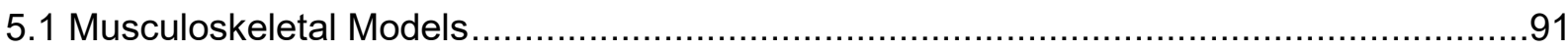




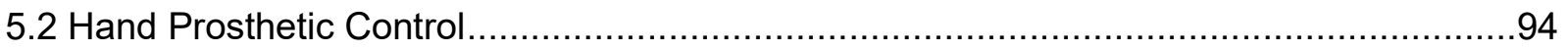

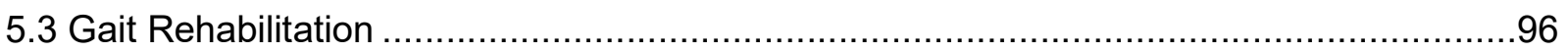

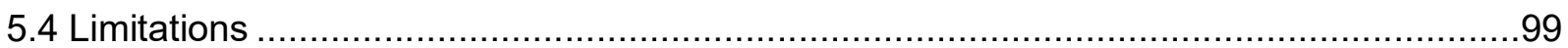

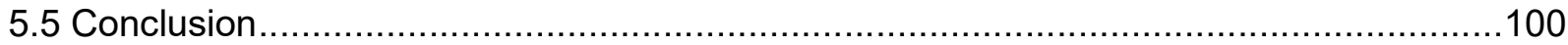

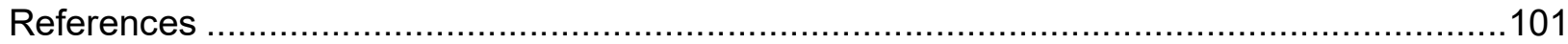




\section{List of Figures}

Fig. 2-1: Upper-limb model of human arm and hand in OpenSim ..................................22

Fig. 2-2: The volume of required musculoskeletal measurements for model validation ............26

Fig. 2-3: The process of structural validation using an experimental dataset ........................28

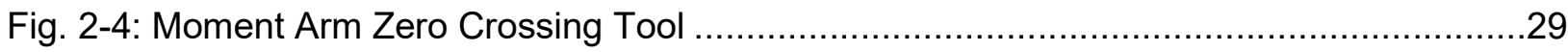

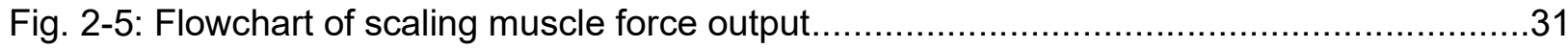

Fig. 2-6: The categorical comparison of structural quality between models with and without

structural validation plotted as categories for all DoFs and all muscles ............................33

Fig. 2-7: Histograms of muscle moment arm zero-crossing events as a function of position. ....34

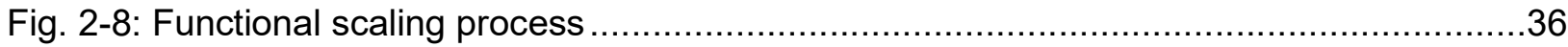

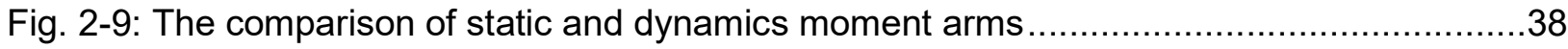

Fig. 3-1 Prosthetic Hand Biomimetic Controller .........................................................48

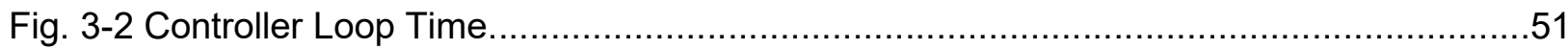

Fig. 3-3 Group Muscle Signal Processing Example Schematic ...................................55

Fig. 3-4 Desired and Simulated Joint Angle Comparison for Gaussian Signals ......................57

Fig. 3-5 HVE Clusters for Gaussian Signals and Recorded EMG .....................................59

Fig. 3-6 Comparison of EMG and Gaussian Hierarchical Clusters .................................60

Fig. 3-7 EMG Driven Control with Muscle Group Processing ..........................................62

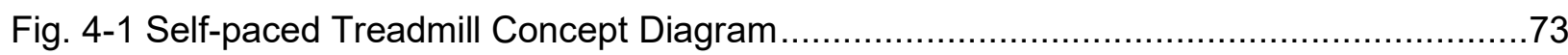

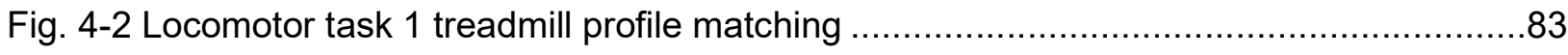

Fig. 4-3 Locomotor task 2 individual leg speed control ............................................. 85

Fig. 4-4: Asymmetric locomotion in VR using self-paced split-belt treadmill with Headset or On-

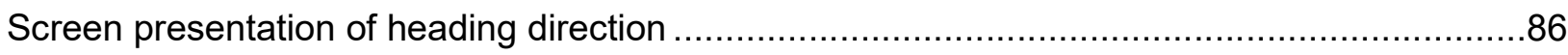

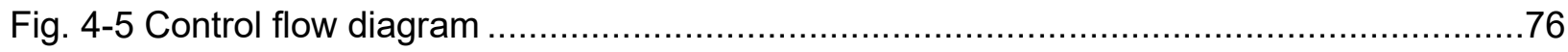

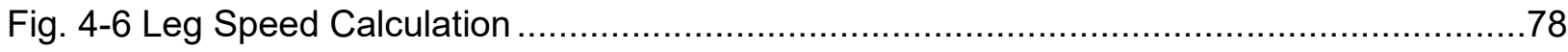




\section{List of Abbreviations}

$\mathrm{AU}$ : arbitrary units

CMC: carpometacarpal

CNS : central nervous system

CPG : central pattern generator

$\mathrm{C}_{\mathrm{F}}$ : scaling coefficient

DOF : degree of freedom

EMG : electromyography

$\mathrm{F}$ : muscle force

HVE : heterogeneous variance explained

$\theta$ : joint angle

$\theta_{m}:$ joint mapped

$\theta_{s}:$ joint sensor

$\mathrm{L}$ : muscle length

$\mathrm{R}$ : muscle moment arm

$r$ : correlation coefficient

MCP : metacarpophalangeal

MS : musculoskeletal

MSD : musculoskeletal dynamics

MVC : maximum voluntary contraction

PCSA : physiological cross-section area

RMSE : root mean square error

ROM : range of motion

SD : standard deviation

$\tau:$ muscle torque 


\section{Chapter 1 Introduction}

Movement is the only way that people can interact with the world around them. Damage to the motor system has an impact on a person's quality of life, affecting both their physical and psychological health (Graczyk et al., 2018; Laurent et al., 2011). People turn to restorative robotics to help them recover from their injury and regain functionality. Depending on the damage, there is a wide range of solutions to help restore limb functionality, from prostheses that replace lost limbs to rehabilitation techniques and orthotic devices. An example of such a device is an epidural electrical stimulator that augments the diminished locomotor function in people with spinal cord injuries (Carhart et al., 2004; Wagner et al., 2018). Even though in Wagner's work a healthy walking gait pattern was not restored, those people could walk again with the assistive device (e.g., a walker). There have been better gait pattern restoration from prostheses to below-knee amputees (Au et al., 2008, 2007; Grimmer et al., 2016); however, when the prosthesis gets more complex, such as a 6 degrees of freedom (DOF) prosthetic hand (Resnik et al., 2018a), the desired natural control is harder to achieve (Cordella et al., 2016).

Human movement, although an intuitive function, is an overwhelmingly sophisticated control problem. In the classical work, Bernstein posed this as a problem of motor redundancy, where the same movement can be generated in a variety of ways to obtain the same goal (Bernstein, 1967; Domkin et al., 2002). This high-dimensional solution space is a challenge for machine learning, where the required amount of data and training time needed increase together with the mechanical complexity of prostheses. With a simple prosthesis, 1 to 2 DOFs, the control problem is relatively simple, but as the number of DOFs increases further, it becomes a hurdle that machine learning approaches struggle to solve.

Human-machine interfaces can be improved by adding solutions from biological systems, e.g., embedding physiological processes into the control structure. This method is termed the 
biomimetic approach, and it has been demonstrated to succeed in prosthetic applications (D. Crouch and Huang, 2016a; Pan et al., 2018a; Sartori et al., 2018). The nonlinearities of the musculoskeletal system can be approximated with models, which would then simplify the transformation from the input muscle activations to the output representing prosthetic movement. This type of controller would not require a human to learn the nonlinearities of the controlled device; instead, the control would feel intuitive and require minimal learning. I have developed and applied the biomimetic approach to two different targets of restorative robotics, upper-limb (see Chapters 2 and 3) and lower-limb movements (see chapter 4). These two targets were chosen to show that the biomimetic approach can be applied broadly to humanmachine interfaces. The following sections provide background knowledge for the approaches used in the dissertation.

\section{Muscles and Electromyography}

All movement is performed through the neuromuscular system. The nervous system sends signals from motor neurons in the spinal cord to muscle fibers. The motor neuron and the muscle fibers it innervates are called a motor unit. A muscle consists of thousands of muscle fibers organized into a smaller number of motor units. Each muscle fiber can be broken down into a set of parallel myofibrils, which are a series of sarcomeres separated by z-disks where each sarcomere is a thick muscle filament surrounded by thin filaments. The thin filaments are anchored to z-disks at each end of the sarcomere. The force produced by each sarcomere comes from the sliding interaction of the contractile thick and thin filaments. When a muscle is activated, the thin filaments slide along the thick filament, and the length of the sarcomere is changed. This process is called the sliding filament theory, which states that each sarcomere shortens as the thin filaments slide closer together between the thick filament so that the z-discs are pulled closer together. The amount that the thick and thin filaments overlap relate to the length of the muscle and the amount of force that each sarcomere can produce, with the 
maximum happening at the muscles rest length (optimal overlap) and the minimum happening when the muscle is shortest or longest (poorest overlap). This process describes the forcelength relationship of the muscle force.

Muscle contraction is caused by the motor neurons discharging action potentials that travel to the muscle through the neuromuscular synapse, causing a motor unit action potential to propagate along the sarcolemma. These action potentials cause the release of calcium ions within the sarcoplasm of the muscle fiber that triggers the myosin heads on the thick filament to bind to actin on the thin filaments. The myosin head actions are regulated by the cross-bridge cycle, which is a sequence of detachment, activation (power stroke), and attachment. During the power stroke, the myosin heads pull the thin filaments along the thick filament shortening the sarcomere and producing muscle contraction. The maximal rate that a muscle fiber can shorten is limited by the peak rate of the cross-bridge cycle, which limits how fast a muscle can contract. The speed of muscle contraction affects the amount of force that the muscle can produce, where the peak force declines as the rate of shortening increases. The force is reduced as the contraction speed increases due to there being fewer myosin heads pulling the thin filaments at one instance as they are trying to detach and reattach quickly. This process describes the forcevelocity relationship of the muscle force.

The analysis of forces that produce a movement is a problem that requires the mechanical properties of muscles. These mechanical properties have been described in muscle models, and since the classical work by Hill (Hill, 1938), there has been a wide variety in the complexity of muscle models. Winters and Stark (Winters and Stark, 1987) separated muscle models into three main groups. The first group consists of second-order models, where the muscle and joint system is treated as a "black box" in which the contents of the box are approximated by a second-order linear system. The second group consists of Hill-based Lumped-Parameter models. This model is based on the structural model developed by A. V. Hill (Hill, 1938) and 
consists of a "contractile element" in series with a series viscoelastic element (Kelvin model) (Romero and Alonso, 2016; Winters and Stark, 1987). A mathematical description of the Hilltype muscle model is described in section 3.2.1 equations 3-1 to 3-4. The last group is Huxleybased distributed-parameter models. These models attempt to explain the contraction mechanism with high accuracy but at a high computational cost. Today, the Hill-type muscle models are the most commonly used for biomechanical studies (Ackermann and Schiehlen, 2006; Romero and Alonso, 2016; Thelen, 2003; van Soest and Bobbert, 1993; Yakovenko et al., 2004; Zajac, 1989). The active element of the Hill-type muscle model relies on the force-lengthvelocity relationship and the maximum muscle force parameter modulating the muscle activation input signal (Zajac, 1989). The force-length relationship describes the amount of force that can be produced based on muscle length. The muscle length is created from the sarcomere lengths, which relate to the overlap of the thick and thin filaments and how many myosin heads can be attached to create the force. The force-velocity relationship describes the force production based on contraction speed, which relates to the myosin heads attaching and detaching quickly and limiting the number attached at one instance. The faster the contraction, the less force that can be produced. The maximum muscle force parameter is estimated based on the muscle physiological cross-section area (PCSA) and specific tension. Muscle activation is a value between 0 (no activation) and 1 maximum activation, and it can be estimated based on electromyography data.

Electromyography (EMG) is the process of measuring the motor unit action potentials, also referred to as muscle activity. This activity can be recorded epidermally, called surface EMG, or via inserted needles, called intramuscular EMG. The timing and amplitude of the EMG signal reflect the activation of muscle fibers by the motor neurons. With surface sensors, the signal is a summation of the local motor unit action potentials occurring under the skin. These action potentials occur at different intervals, and at any one instance, and span positive or negative 
voltage values. The different signs result from the difference in directions that the motor unit action potentials propagate relative to the electrode orientation. The detection of a single motor unit action potential is possible with intramuscular EMG sensors, where a needle is placed directly in the muscle. Single units are also detectable with a surface EMG method (Farina et al., 2008; Miller et al., 2014). More recently, electrode grids have been used for detecting the propagation of single motor unit action potentials. Surface EMG sensors can have issues with cross-talk, which is when an EMG signal is contaminated by the activity of neighboring muscles. This problem can be detected by, e.g., cross-correlation analysis and mitigated by the commonmode rejection or sensor repositioning. To evaluate the sensor placement, a participant performs single joint degree of freedom (DOF) movements, and the recorded data is reviewed to see if sensors detect expected activity during the different movements. This information is used to determine the recruitment of muscles as well as to evaluate the cross-talk. Intramuscular EMG is less influenced by cross-talk, but the sampled muscle volume can be small and not representative of the whole muscle. The processing performed on EMG is done to remove electrical noise and movement artifacts as well as determine the activity of the muscle. The first step of the process is to high-pass filter the data with a cutoff frequency between 10-30 $\mathrm{Hz}$ (De Luca et al., 2010) to remove any low-frequency noise caused by electrode drift or motion artifacts. The second step is to demean the data, then perform full-wave rectification. The rectification is performed so that the signal does not average to zero due to the signal bouncing from positive to negative values. The last step is to low-pass filter the data with a cutoff anywhere from 5 to $100 \mathrm{~Hz}$ to remove additional noise. The processed EMG signals show an envelope of the muscle activity that can be used to estimate the recruitment of the muscle, which can be used as an input to control a prosthesis. 


\section{Upper Limb Prosthetic Control}

Advanced complex limb prostheses and exoskeletons are becoming tools that can restore lost functionality or augment natural abilities. Advancements in mechatronics have generated new prosthetic hardware that is expanding the functional movements to be more realistic similar to that of an intact human arm (Carrozza et al., 2006; Kyberd et al., 2001; Resnik et al., 2018a). More functional machines should have a high adoption rate among amputees. Yet, people choose simple, often passive prostheses. The major bottleneck to widespread adoption is associated with the difficulty in controlling the devices and their lack of robustness. Around 10 to $25 \%$ of upper limb amputees do not use any prostheses (Berke et al., 2010; Biddiss and Chau, 2007; Pinzur et al., 1994; Raichle et al., 2008), and only half of the remaining choose to use a powered prosthesis (Whiteside et al., 2000). A popular choice is a body-powered prosthetic gripper, where opening and closing are controlled by whole arm movements. The current

myoelectric prostheses operated by muscle activity lacks robustness to be widely adopted (Cordella et al., 2016).

The commonly used clinical approach for using myoelectric signals is direct control, which links an agonist-antagonist muscle pair to move a prosthetic DOF (Ison and Artemiadis, 2014) with the movement speed being proportional to the muscle activation (Scott and Parker, 1988; Williams, 1990). This approach works for controlling a 1-DOF prosthesis, but it becomes cognitively demanding when there are 2 or more DOFs. A switching technique is used to enable the control of more than one DOF and allows the amputee to switch the DOF controlled by using muscle cocontraction or a mechanical switch (Schultz and Kuiken, 2011). These myoelectric prostheses have become clinically adopted; however, they do not typically allow the control of more than one DOF at a time (Cordella et al., 2016). 
There are many methods that attempt to extract user intent from EMG. The most common is based on detecting the relationship between EMG and movement through pattern recognition. (Birdwell et al., 2015; Graupe et al., 1977; Scheme et al., 2014; Scheme and Englehart, 2011; Smith et al., 2014). The goal is to create a classifier that can associate EMG activity with a specific movement (e.g., wrist supination, wrist pronation, hand grasp, hand open, etc.). This process requires the collection of training data for each movement and periodic recalibration. When the process works, the user moves their prosthesis naturally, moving multiple DOFs without a cognitive load just like they move their intact hand. One of the downsides of the approach is that it works well for the posture it was trained in but has reduced functionality in other limb postures. Because of this downside, it has been shown that pattern recognition controllers are not always more intuitive and straightforward for the user than the direct control method (Resnik et al., 2018b). In the study, an amputee controlled a 2 DOF prosthesis (i.e., wrist pro/sup and gripper open/close) with two different controllers, direct control and pattern recognition. Even though there was a cognitive load with direct control, they were able to perform better or the same as the pattern recognition method on functional tasks such as the box and block test. One of the main issues of decoding the movement intent is that there is a nonlinear relationship between the EMG signals and the movement due to the muscle physiology (Dantas et al., 2019). What could potentially change the landscape of this status quo is the use of a biomimetic approach.

Our solution for going from EMG to motion is to use both muscle models and musculoskeletal dynamics to translate EMGs into the intended movement. The approach works by estimating muscle recruitment from EMG and calculating forces from muscle models. The cross-product of moment arms and these forces computes muscle moments for each DOF. The sum of moments is the muscle torque calculated for each DOF in the model. The internal and external torques for each DOF are inputs in the equations of motion that are numerically simulated by a physics 
engine to compute the arm and hand movement. This approach accounts for some of the nonlinearities in the EMG-to-motion transformation by modeling the intrinsic muscle dynamics that cause non-linearities (e.g., muscle force-velocity relationship). The general idea is supported by the evidence of embedded MS dynamics within the neural computations of planning and execution pathways (Lillicrap and Scott, 2013; Shadmehr et al., 2016). This approach can be used as the principle for prosthetic control (D. Crouch and Huang, 2016a; Sartori et al., 2018). There have even been simultaneous 2 DOF control using this method with constant moment arms, where hand grasping was controlled by agonist-antagonist finger muscles, and wrist flexion/extension was controlled by agonist-antagonist wrist muscles (Crouch et al., 2018; Pan et al., 2018a). This method has the capability to control multiple DOFs simultaneously and would complement new intricate prosthetic designs.

With the number of upper limb amputees expected to at least double from 2005 to 2050 (Ziegler-Graham et al., 2008), there is a need for developing better and more robust prosthetic controllers.

\section{Musculoskeletal Modeling Validation}

The popular truism, "all models are wrong, but some are useful," expressed by a statistician George Box (Box, 1979), guides the acceptable validity in ever more ambitious model developments. This statement suggests a strategy in model development defined by strict adherence to its usefulness. Multiple models can be developed and have only limited use: 1)

they could be inaccurate representations of simulated transformations or 2) the implementation could be too slow for practical application. A musculoskeletal (MS) model consists of the geometrical description of the muscle paths around the skeleton, which describes the points of attachment and path around the joints for each muscle in the model. For a human arm, the muscle paths, on average, cross 3 degrees of freedom (DOFs) and have multiple muscles with 
multiple points of attachment (i.e., flexor digitorum, extensor digitorum, pectoralis major, etc.). Extensor Pollicis Longus (EPL) spans over 6 DOFs (i.e., wrist sup/pro, wrist flex/ext, thumb CMC flex/ext, thumb CMC abd/add, thumb MCP flex/ext, and thumb IP flex/ext), and only sparse experimental data exists describing the actions about these DOFs. The best practice is the operational development of models based on experimental measurements, using minimalistic sets of parameters and additional testing of model performance (Kirchner, 2006). The sparseness of experimental structural and functional datasets is the main limiting factor in model development. In the context of MS models used within artificial limb control algorithms, the challenge is to develop a valid representation of reduced morphological and behavioral relationships between muscles and physiological postures. This process typically involves approximating the relationships to reduce the computation time for real-time applications (Menegaldo et al., 2004; Sartori et al., 2012; Sobinov et al., 2019). In our efforts to achieve robust models of hand control, we leveraged the use of real-time computations to enable the development of models with robust performance over the full range of physiological postures.

The effort to create valid muscle-driven MS models spans dozens of years in the context of noninvasive analysis of gait, posture, and reaching movements (Arnold et al., 2010; Carbone et al., 2015; Delp et al., 1990; Gritsenko et al., 2016; Horsman, 2007; Rajagopal et al., 2016; Saul et al., 2015b). In general, MS models are tested through either direct or indirect validation (Henninger et al., 2010; Lund et al., 2012). Comparing simulated and measured muscle attributes like moment arms is an example of a direct morphological validation (Arnold et al., 2001, 2000; Delp et al., 1990; Holzbaur et al., 2005). An example of indirect validation is the comparison of simulated and recorded muscle activity patterns that produce the same movement (de Zee et al., 2007). A direct comparison is preferred; however, there is limited availability of muscle moment arm data for different postures. Often, MS models rely on disparate data sources, i.e., measurements combined across cadavers and different studies 
that may use different methodologies. The combined models may inherit inter-subject variations based on measurements that are not correctly scaled and lead to unphysiological nonlinearities (Goislard De Monsabert et al., 2018). This problem necessitates the use of additional indirect validations that examine overall function. For example, scaling forces of individual muscles by either changing their force generation parameters (Scovil and Ronsky, 2006) or their muscle moment arms (Nussbaum et al., 1995) to match observed torque measurements.

\section{Locomotion Central Pattern Generators}

We have volitional cortical control of gait pattern, direction, and velocity, but the autonomic spinal control is a significant part of the locomotion. This was first demonstrated in 1911 when Graham T. Brown observed that cats could still walk on a treadmill if their body weight was supported even after their spinal cord and dorsal roots, the source of sensory inflow, were cut (Brown, 1911). Based on the lack of descending input or sensory feedback, he suggested that the rhythmic activity was produced in the spinal cord itself by some kind of internal motor circuit, which came to be known as a central pattern generator (CPG). Over the years there have been many other experiments pointing towards a spinal locomotor CPG (Brown, 1911; Grillner, 2006; Grillner et al., 1976; Grillner and Zangger, 1975; Kiehn, 2006; Lundberg and Phillips, 1973; Meehan et al., 2012; Shik et al., 1969; Zhong et al., 2012). In the 1930s, Brown shows that a decerebrated cat can walk and gallop with only sensory feedback (Lundberg and Phillips, 1973), which means that spinal circuitry can compensate for the lack of cortical inputs for basic locomotion. Here, the change of treadmill speed actuated the CPG through sensory pathways, the phenomenon known as the neuromechanical tuning. Another study showed that stimulating the mesencephalic locomotor region of the midbrain in a decerebrated cat allowed it to go from walking to galloping based on the stimulation frequency and intensity (Shik et al., 1969). There has even been work where the spinal cord was isolated in vitro and shown to contain networks that can produce fictive locomotor patterns spontaneously or when stimulated electrically or 
chemically (Meehan et al., 2012). All the studies above demonstrate that the locomotor CPG contributes to the following three main locomotor features: i) the rhythmic and cyclical behavior; ii) coordination between antagonistic muscles in the same limb; and iii) alternating patterning between opposite limbs.

CPGs are found in different areas of the central nervous system and are responsible for different types of rhythmic activities such as breathing, chewing, swallowing, and walking. These activities are run by spinal interneurons that interconnect to form CPG networks that produce rhythmic firing. The first organization of the locomotor CPG was described by Brown (Brown, 1914) and named the "half-center" model. The model described two flexor and extensor halfcenters pairs, one for each leg, that interactively inhibited each other. The interactions between the half-centers were controlled by inhibitory interneurons so that only one half-center in a pair could be active at a time. The activity of the half-center would gradually fatigue and lead to the activation of the antagonistic half-center, which caused the locomotor phase to switch. This classical half-center model represents a general and simplified CPG organization that cannot describe some features of the real locomotor CPG, such as how the locomotor pattern does not exhibit strict alternations between all flexor and extensor motorneurons. To overcome this issue and others, Grillner (Grillner, 1981) proposed a new organization for the locomotor CPG that used a unit burst generator concept that suggested the existence of separate rhythmic generators controlling joint movement with one for every joint movement direction. Then each burst generator was coupled together to produce multijoint coordination. There is another solution developed by Rybak (McCrea and Rybak, 2008; Rybak et al., 2006), which suggested that the spinal CPG has an organization with two levels consisting of a bipartite half-center rhythm generator and pattern formation circuits. Each of the different models was developed and used to help further our understanding of spinal CPGs and the interneurons that connect to form them. There are multiple CPG models, with each having a different level of complexity 
involved to implement that model. For example, the unit burst generator and two-level CPG organization are more complex models than the half-center model. The more complex a model, the harder it is to keep errors from creeping in. One of the main challenges is choosing the appropriate model for a given problem, and it involves deciding on the model complexity and level of abstraction needed to solve the required task.

The benefit of having a CPG responsible for the generation of a repetitive task is that it reduces the complexity of the required descending command to perform the task by offloading some control tasks to local autonomic controllers (Pfeifer et al., 2007). The general question that remains is what drives these spinal CPGs? In 2011, Yakovenko (Yakovenko, 2011) inversely solved the Brown half-center model and found a linear relationship between the locomotor CPG input and limb velocity. This result is consistent with multiple experimental observations (Collins and Richmond, 1994; Ivanenko et al., 2000; Shik et al., 1969). The classical work by Shik et al. in 1969 supports this theory with their observations made by stimulating the mesencephalic locomotor region in cats (Shik et al., 1969). In that study, they reported that a ramping increase in the stimulation produced a continuous transition from walking to galloping. The speed of locomotion in humans has been shown to be increased by the mechanical stimulation of primary afferents (Ivanenko et al., 2000). Both of these studies show that by increasing feedforward or feedback inputs to the CPG, the speed of locomotion could be increased. Furthermore, the theoretical studies of asymmetric locomotion (Sobinov and Yakovenko, 2018; Yakovenko, 2011; Yakovenko et al., 2018) using experimental data (Halbertsma, 1983) suggest the ability to control limb speed. In complementary studies of human curved-path locomotion, the individual limb speed control translates into the control of heading direction (Hase and Stein, 1999). Two strategies of turning have been suggested in this context-step and pivot turning-supporting the use of limb speed for slow turning of about $20^{\circ}$ per second and requiring hip rotation for the 
abrupt pivoting turns. The ability to forward and inversely solve the locomotor CPG can lead to new insights into the development of different technologies to analyze locomotion.

\section{Gait Rehabilitation}

Locomotor impairments lower the quality of life, and their rehabilitation is imperative for independent living. The prevalence of disabilities increases with age (Taylor, 2018), and the most common is a mobility impairment. Roughly $13.7 \%$ of all adults in the United States have a mobility impairment and $26.9 \%$ of all people aged 65 and older have one (Okoro et al., 2018). The population of people aged 60 and older is expected to increase by about $50 \%$ in the next 10 years, with them accounting for around $25 \%$ of the population (United Nations, Department of Economic and Social Affairs, 2015). The need for gait rehabilitation strategies and technologies is expected to increase (Mikolajczyk et al., 2018).

Developing better rehabilitation technology and techniques could improve recovery rates. Basic physical therapy involves overground walking therapy (i.e., walking with the help of a physiotherapist and horizontal parallel bars), muscle strength training, and transferable skills training (e.g., sitting to standing) (Mikolajczyk et al., 2018). Bodyweight support treadmills have been proposed and gained attention due to the benefit of allowing a person to walk early in their rehabilitation and complete gait cycles safely without a lot of physical exertion (Sousa et al., 2009). The downside of the treadmill walking is that it is currently unclear if differences in treadmill walking make it hard to learn and retain healthy gait patterns to overground walking. There has been a multitude of studies comparing overground and treadmill walking with conflicting results (Alton et al., 1998; Lee and Hidler, 2008; Riley et al., 2007; Stolze et al., 1997). Overall the studies state that treadmill walking is similar to overground walking with only a couple agreed upon differences, the main one being that treadmill walking induces a slower 
preferred speed (Lee and Hidler, 2008; Plotnik et al., 2015). The primary suggested reasons for the differences are the absence of visual flow and the imposed fixed speed of the treadmill.

To mitigate these potential problems, virtual reality linked to self-paced treadmills was developed to provide visual flow on screens (Plotnik et al., 2015; Sloot et al., 2014a). These studies have shown benefits from systems that allow a person to walk at self-selected speeds with the proper visual flow. Plotnik et al. showed that after 50 meters, people walking on a selfpaced treadmill would walk at the same speed as they do overground; however, if the visual flow is added, they walk at a comparable speed immediately (Plotnik et al., 2015). Sloot et al. showed a similar result where visual flow increased walking parameters to be closer to overground walking (Sloot et al., 2014a). Interestingly, it has even been shown that walking on a single belt treadmill with turning visual flow can elicit physical turning behavior (e.g., head-

turning and shortening stride length) that matches overground walking (Oh et al., 2018). Current self-paced treadmills do not allow interlimb speed differences that would allow turning behavior. Previous studies demonstrated that interlimb speeds vary during turning (Courtine et al., 2006; Patla et al., 1985), so without this feature, there cannot be any turning. Typically, self-paced behavior is enabled by keeping the subject's position in the middle of treadmill using an attached depth sensor (Jonghyun Kim et al., 2013; Minetti et al., 2003), rope anchor (Lichtenstein et al., 2007), or motion capture (Sloot et al., 2014b; Souman et al., 2011; Stavar et al., 2011). These solutions allow whole-body speed adjustments without the dexterity of individual limb control.

\section{Dissertation Summary}

In my dissertation, I used a biomimetic approach to target two different applications in restorative robotics, one involving the upper limb (prosthetic hand control) and one involving the lower limb (self-paced treadmill for gait rehabilitation). The overall goal was to use a biomimetic 
approach to improve the control robustness and the intuitiveness of human interfaces with restorative robotics.

Chapters 2 and 3 describe the control of prosthetic hands with a musculoskeletal model. In Chapter 2, I described a method for musculoskeletal model validation and tools to help ensure the model provides a holistic representation. I performed this validation process on a musculoskeletal model of a human arm. I also investigated what is lost if the musculoskeletal dynamics are simplified to constant moment arms, which has been previously done for prosthetic control (Crouch et al., 2018; Pan et al., 2018a). The main impact of this work is the development of the validated musculoskeletal forearm and hand model. In chapter 3, I describe how we used a musculoskeletal model along with a Hill-type muscle model to calculate the intended movement and control different prostheses. Additionally, I show the feasibility of using Gaussians signals for simplified control signals and describe a method to infer muscle activity for muscles not recorded inspired by a muscle's function. This main contribution of this work is the method that uses the full description of a musculoskeletal model to decipher movement intent and use it for control.

In chapter 4, I switch to my lower limb work that describes the development of a self-paced treadmill system that operates like an inverse locomotor CPG. The system is supplied phase and swing transitions and outputs the current leg speed. Limb speeds are independently calculated, allowing self-paced control for each treadmill belt. Moreover, adding the transformation from limb speeds to the heading direction using virtual reality (VR) changes the direction of walking and constitutes the omnidirectional treadmill. This transformation was observed in humans as the step strategy where the inner limb is slower than the outer limb in walking on a curved path (Hase and Stein, 1999). The strategy was shown to work for the changes in heading direction exceeding original expectations. This concept was implemented and tested with healthy participants. The main impact of this work is the self-paced treadmill 
with independent limb control. It allows omnidirectional movement in virtual reality as well as a system that could be used to improve gait rehabilitation. 


\section{Chapter 2 Validating a Realistic Musculoskeletal Model for}

\section{the Arm and Hand}

(this chapter is in preparation to be submitted to Scientific Reports as "Boots M, Sobinov A, Hardesty R, Gritsenko V, Gaunt R, et al., Yakovenko S (2019) Realistic musculoskeletal models for real-time human-in-the-loop control of arm and hand")

\subsection{Abstract}

Models of musculoskeletal structure and function can be used in the biomimetic control of artificial limbs. For these models, their parameters representing posture-dependent moment arms and force generation are estimated over the full physiological range of motion. However, available experimental measurements are sparse and often disparate. The required model validation is not trivial for high-dimensional representations attempting to capture the behavior of human limbs. In this study, we developed a method to validate and scale kinematic muscle parameters using posture-dependent moment arms profiles and isometric measurements for whole-limb force generation. We used an OpenSim model (Gritsenko et al., 2016; Saul et al., 2015a) with 18 degrees of freedom (DOFs) and 32 musculotendon actuators with force generated from a Hill-type muscle model (Yakovenko et al., 2004). The geometry a muscle takes from origin to insertion was used to model the muscle lengths and moment arms. We simulated moment arm profiles across the full physiological range of motion and compared them to published experimental data, which were digitized and averaged. The validation process identified the quality of performance between simulated and experimental data using the rootmean-square and correlation coefficient of the difference between simulated and experimental values. Since the available measurements were sparse, additional computed metrics were utilized to identify common failures, i.e., sign flipping in simulated moments and the imbalance 
of force generation between antagonistic groups in postural extremes. To account for the assembly errors due to different types of experimental measurements, we scaled the force generation of agonistic groups acting around 8 representative DOFs. The model simulated posture-dependent maximum voluntary contraction measurements with high accuracy. Finally, we used this model to show the consequences of choosing constant instead of the posturedependent moment arms on torque generation. We found that there was a reduction of joint torques by $34.9 \%$ in the extreme quartiles of the wrist flexion-extension DOF. The structural details embedded in realistic musculoskeletal models may improve the understanding of muscle actions and help in the design and control of artificial limbs.

\subsection{New \& Noteworthy}

Realistic models of human limbs are a development goal required for the understanding of motor control and its applications in biomedical fields. Here, we have overcome the challenge of sparse experimental data by using multi-stage validation, relying on measurements and simulations to identify failures in the high-dimensional structure of muscle paths that span up to 6 dimensions. We demonstrate that these details are highly relevant to the understanding of force generation at the wrist. 


\subsection{Introduction}

Movement is a fundamental behavior of all living organisms allowing them to manipulate the external environment to achieve survival objectives. Although moving is not generally considered to be cognitively demanding, humans solve the challenging problem of accurately controlling high-dimensional musculoskeletal (MS) machinery in real-time. By comparison, the relatively simpler control of artificial limbs is far from its biological counterpart; the sophisticated algorithms currently are only solving simple mechanics (D. Crouch and Huang, 2016a; Dantas et al., 2019; Resnik et al., 2018b). One possible solution of this engineering problem is to simplify the control problem with the use of internal models, as in the Smith's predictor (Smith, 1957); and it is supported by the evidence of embedded MS dynamics within neural computations of planning and execution pathways (Lillicrap and Scott, 2013; Shadmehr et al., 2016). This general approach can be potentially used as the principle for prosthetic control (D. Crouch and Huang, 2016a; Sartori et al., 2018); however, capturing accurate structural and functional dynamics of the human body in MS models remains a significant challenge.

The popular truism, "all models are wrong, but some are useful," expressed by a statistician George Box (Box, 1979), guides the acceptable validity in ever more ambitious model developments. In the context of MS models used within artificial limb control algorithms, the challenge is to develop a valid representation of reduced morphological and behavioral relationships between muscles and physiological posture. The effort to create valid muscledriven MS models spans dozens of years in the context of non-invasive analysis of gait, posture, and reaching movements (Arnold et al., 2010; Carbone et al., 2015; Delp et al., 1990; Gritsenko et al., 2016; Horsman, 2007; Rajagopal et al., 2016; Saul et al., 2015b). The best practice is to development of models based on experimental measurements, using minimalistic sets of parameters and additional testing of model performance (Kirchner, 2006). The 
sparseness of experimental structural and functional datasets is the main limiting factor in model development.

In general, MS models are tested through either direct or indirect validation (Henninger et al., 2010; Lund et al., 2012). Comparing simulated and measured muscle attributes like moment arms is an example of a direct morphological validation (Arnold et al., 2001, 2000; Delp et al., 1990; Holzbaur et al., 2005). An example of indirect validation is the comparison of simulated and recorded muscle activity patterns that produce the same movement (de Zee et al., 2007; Hamner et al., 2010). A direct comparison is preferred; however, there is limited availability of moment arm data for different postures. Often, MS models rely on disparate data sources, i.e., measurements combined across cadavers and different studies that may use different methodologies. The combined models may inherit inter-subject variations based on measurements that are not correctly scaled and lead to unphysiological nonlinearities (Goislard De Monsabert et al., 2018). This problem necessitates the use of additional indirect validations that examine overall function. For example, scaling forces of individual muscles by either changing their force generation parameters (Scovil and Ronsky, 2006) or their muscle moment arms (Nussbaum et al., 1995) to match observed torque measurements.

In this study, we aimed to overcome structural and functional model inaccuracies by applying a novel direct structural validation method combined with an indirect validation of functional output. We improved the quality of the previous realistic arm and hand model hand (Gritsenko et al., 2016; Saul et al., 2015a) and tested the potential errors in torque generation in simplified models with constant moment arm profiles. We investigated the simplification of dynamic moment arms to constant because changes in the moment arm profiles could amount to substantial differences in the torque production at the joint. 


\subsection{Methods}

\subsubsection{Model}

We used a model of an arm and hand (Gritsenko et al., 2016) developed in OpenSim (Delp et al., 2007). In Gritsenko et al., we modified the Saul et al. model (Saul et al., 2015a) to include separate segments of the hand digits, adding an additional 16 degrees of freedom (DOFs). The model simulated 52 musculotendon actuators representing 32 muscles spanning 23 DOFs in total. To simplify the model, we excluded abduction (abd) / adduction (add) DOFs of the second to fifth digit metacarpophalangeal (MCP) and a wrist abd/add DOF. To improve the thumb movement description, we added two intrinsic hand muscles, opponens pollicis and flexor pollicis brevis.

In this study, we removed the muscles that span only the shoulder and elbow and added intrinsic thumb muscles (abductor pollicis brevis and adductor pollicis) to describe 33 musculotendon actuators representing 24 muscles of the distal arm and hand, shown in Fig. 2-1. The muscle paths in the current model were further modified using an MS validation process (see below, Validation Process). This process can be summarized as the recurrent adjustment of all the muscle paths using experimental measurements (see below, Datasets). The model validation process consisted of the following three steps: $i$ ) the creation of a meta dataset describing muscle moment arm values for a sparse selection of postures from published studies, ii) the selection of wrapping geometry to constrain muscle paths, and iii) the validation of structural and functional muscle properties. 


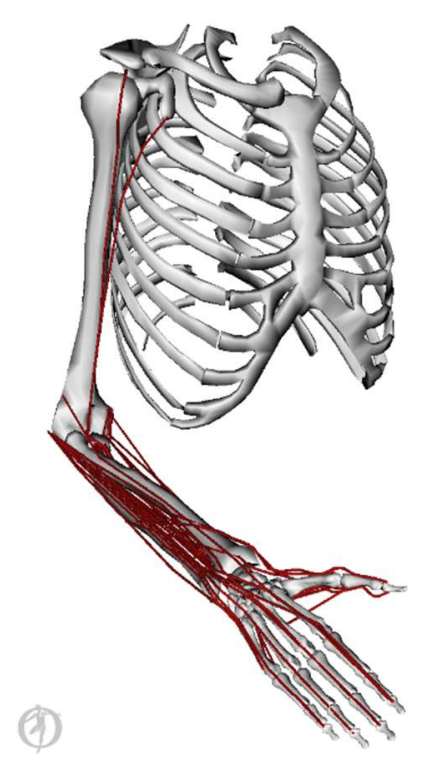

Fig. 2-1: Upper-limb model of human arm and hand in OpenSim. The muscle paths (red) were defined relative to the skeletal landmarks and wrapping geometry.

\subsubsection{Datasets}

Three datasets were required for the structural and functional validation of MS model: i) published muscle moment arm measurements; ii) published torque measurements in maximal voluntary contraction (MVC) tasks; iii) simulated muscle moment arm values. The first dataset was used as the gold standard in the recurrent adjustments of simulated muscle paths of the third dataset. The second dataset was used to scale force generation of multiple agonistantagonist muscles spanning the same joint. The next three sections describe these datasets in detail.

\subsubsection{Dataset 1. Moment Arm Measurements}

The experimental dataset of moment arm measurements was generated as the 'gold standard' for the simulated relationships (Dataset 3). We reviewed all the currently available publications and collated all upper-limb measurements of the relationships between human muscle moment arms and posture. The measurements were not of uniform quality. We selected only 7 sources with measurements in cadavers over other methods, e.g., simulated relationships, to reduce the 
potential problems from combining multiple observations and try to increase dataset consistency (Goislard De Monsabert et al., 2018). If there were duplicate sources with similar methodologies, we selected the source with the most extensive information. Then, the relationships between muscle moment arms and limb kinematic posture, termed muscle-DOF relationships (moment arm values for a DOF a muscle spans), were digitized from high-quality scans of figures using a specialized tool, WebPlotDigitizer (Rohatgi, 2018). An example of a muscle-DOF relationship is the moment arm values for the FDS2 muscle about the index finger MCP joint. The muscle-DOF relationships were scaled to standard SI units and placed into a custom relational database. Our search has not identified any muscle-DOF measurements for the proximal and distal interphalangeal joints (PIP \& DIP) of non-index fingers. The profiles of these 17 muscle-DOF relationships were estimated using index digit moment arms and scaling them by the ratio of moment arms at MCP joint between non-index and index homologous muscles. For example, extensor digitorum moment arm profiles about the PIP joint of the middle finger (ED3) were copied from the index finger (ED2) and scaled by the ratio of moment arms for these muscles measured at MCP joint. Table 2-1 summarizes meta information for the included 81 muscle-DOF relationships (see Supplementary Materials for each muscle-DOF relationship and its source publication).

Table 2-1: Sources and meta information on moment arm measurements. This table shows all the sources that were used to create the moment arm databased in this study. It provides information about the measuring method and subjects used in each study.

\begin{tabular}{|c|c|c|c|}
\hline Source & Age (yrs) & \# Samples & Measurement Method \\
\hline (Haugstvedt et al., 2001) & $65(41-90)$ & $8(8 \mathrm{M}, 0 \mathrm{~F})$ & cadaver measurement \\
\hline (Bremer et al., 2006) & NP & $1(1 \mathrm{M})$ & $\begin{array}{l}\text { cadaver and epoxy model } \\
\text { measurement }\end{array}$ \\
\hline (Loren et al., 1996) & NP & 5 & cadaver measurement \\
\hline (Koh et al., 2006) & $71(50-90)$ & $11(6 \mathrm{M}, 5 \mathrm{~F})$ & cadaver measurement \\
\hline (Fowler et al., 2001)* $^{*}$ & $29(29)$ & $1(1 \mathrm{~F})$ & MRI Scan \\
\hline (Smutz et al., 1998) & $77(72-84)$ & $7(4 \mathrm{M}, 2 \mathrm{~F}, 1 ?)$ & cadaver measurement \\
\hline
\end{tabular}


(Gonzalez et al., 1997)

NA

NA

computer model

* Source provided subject height $171.5 \mathrm{~mm}$ and weight $63.5 \mathrm{~kg}$

\subsubsection{Dataset 2. Torque Measurements}

We collected published maximum joint DOF torques for 8 DOFs representing 6 hand joints to scale the muscle maximum isometric force $\left(F_{\max }\right)$, which is one of the main parameters responsible for the magnitude of force generation in the Hill-type model (Zajac, 1989). The goal was to collect representative values, not to conduct a meta-analysis study; thus, this information was determined through the examination of a subset of all studies from which four were selected (Table 2-2). We gave priority to studies that demonstrated the isolation of the measured DOF and had a direct measurement of the maximum torque. Each isometric measurement contained not only the maximum torque but also the required corresponding limb posture. To our surprise, we could not identify studies with a direct maximum torque measurement for the MCP phalangeal joints (see Table 2-2), and we used direct force measurements at an estimated posture instead (Shim et al., 2007). The moment arms to the measured forces were estimated from a distance between the force sensor and the MCP joint for each finger (index $7.5 \mathrm{~cm}$, middle $8.0 \mathrm{~cm}$, ring $7.5 \mathrm{~cm}$, and pinky $6.5 \mathrm{~cm}$ ) to compute the joint torques (values noted with an asterisk in Table 2-2). Table 2-2 shows the summary of recorded values and the corresponding meta-information. These torque values are meant to be representative of an average person in their twenties.

Table 2-2: Maximum Torque Data. This table shows the maximum torque information as well as information about the subjects used in the studies.

\begin{tabular}{l|l|l|l|l|l|l|l|l}
\hline Reference & Joint & Direction & $\begin{array}{l}\text { Maximum } \\
\text { Torque } \\
(\mathrm{Nm})\end{array}$ & $\begin{array}{l}\text { Height } \\
(\mathrm{m})\end{array}$ & $\begin{array}{l}\text { Age } \\
(\mathrm{yr})\end{array}$ & $\begin{array}{l}\text { Age } \\
\text { Range } \\
(\mathrm{yr})\end{array}$ & $\begin{array}{l}\text { Weight } \\
(\mathrm{kg})\end{array}$ & Sex \\
\hline $\begin{array}{l}\text { (Decostre } \\
\text { et al., } \\
2015)\end{array}$ & \multirow{2}{*}{ Wrist } & Elex & 14.5 & 1.77 & 25 & $20-29$ & 74.9 & $27 \mathrm{M}$ \\
& & Pro & 4.64 & NP & 30.2 & $23.9-36.5$ & NP & $11 \mathrm{M}$
\end{tabular}




\begin{tabular}{|c|c|c|c|c|c|c|c|c|}
\hline $\begin{array}{l}\text { (Gordon et } \\
\text { al., 2004) }\end{array}$ & & Sup & 4.38 & & & & & $3 F$ \\
\hline $\begin{array}{l}\text { (Bourbonn } \\
\text { ais and } \\
\text { Duval, } \\
1991 \text { ) }\end{array}$ & $\begin{array}{l}\text { Thumb } \\
\text { CMC }\end{array}$ & $\begin{array}{l}\text { Flex Add } \\
\text { Ext Add } \\
\text { Flex Abd } \\
\text { Ext Abd }\end{array}$ & $\begin{array}{l}4.2 \\
3.4 \\
2 \\
1.6 \\
\end{array}$ & NP & 23.4 & $21-25$ & NP & $12 \mathrm{~F}$ \\
\hline $\begin{array}{l}\text { (Shim et } \\
\text { al., 2007) }\end{array}$ & $\begin{array}{l}\text { Index } \\
\text { MCP } \\
\text { Middle } \\
\text { MCP } \\
\text { Ring } \\
\text { MCP } \\
\text { Pinky } \\
\text { MCP }\end{array}$ & $\begin{array}{l}\text { Flex } \\
\text { Ext } \\
\text { Flex } \\
\text { Ext } \\
\text { Flex } \\
\text { Ext } \\
\text { Flex } \\
\text { Ext }\end{array}$ & $\begin{array}{l}3.15^{*} \\
0.78^{*} \\
3.36^{*} \\
0.70^{*} \\
2.1^{*} \\
0.58^{*} \\
1.59^{*} \\
0.48^{*}\end{array}$ & NP & 22.5 & $20.5-24.5$ & NP & $\begin{array}{l}13 \mathrm{M} \\
12 \mathrm{~F}\end{array}$ \\
\hline
\end{tabular}

${ }^{*}$ estimated joint torque values; Flex $=$ Flexion, Ext $=$ Extension, Pro $=$ Pronation, Sup = Supination, $\mathrm{Add}=$ Adduction, $\mathrm{Abd}=$ Abduction $; \mathrm{NP}=$ Not Provided, $\mathrm{M}=$ Male, $\mathrm{F}=$ Female .

\subsubsection{Dataset 3. Simulated Muscle Measurements}

The simulated muscle moment arm and length values were acquired from the OpenSim model in a uniform grid with 9 points per DOF (Sobinov et al., 2019), creating $9^{d}$ unique postures, where $d$ is the number of DOFs a muscle spans. Each muscle was represented by values corresponding to postures, moment arms, and muscle lengths. A muscle can span multiple DOFs (on average 3), creating $d$ moment arms per posture, $d^{*} 9^{d}$ total moment arm values per muscle. The postures were represented by the same number of values, and the muscle length values defined across DOFs were only one per posture $\left(9^{d}\right)$. Then, the total number of values for each muscle was $9^{d}(2 d+1)$, e.g., an average muscle with $d=3$ was described with $3^{*} 9^{3}$ moment arms, $3^{*} 9^{3}$ postures, and $9^{3}$ muscle lengths for a total of 5,103 values. Fig. $2-2$ gives an idea of how the number of points can increase dramatically for more complex muscles. The left of the figure shows how there are usually values in the physiological range that are not measured. Looking at the central figure, it shows how those points not measured increase as the number of DOFs increases. The right figure shows how the number of points extrapolates as more DOFs are added. The most complex muscle (i.e., EPL) with $d=6$ was captured with 
$6,908,733$ values. The dataset was updated every time a muscle path in the model was adjusted (see below, "Step 1. Structural Validation"). The accurate polynomial approximations (errors less than $1 \%$ of magnitude) (Sobinov et al., 2019) were compared against the published experimental values during structural validation (Step 1, below) and used in the estimation of forces (Step 3) for any given physiological posture.

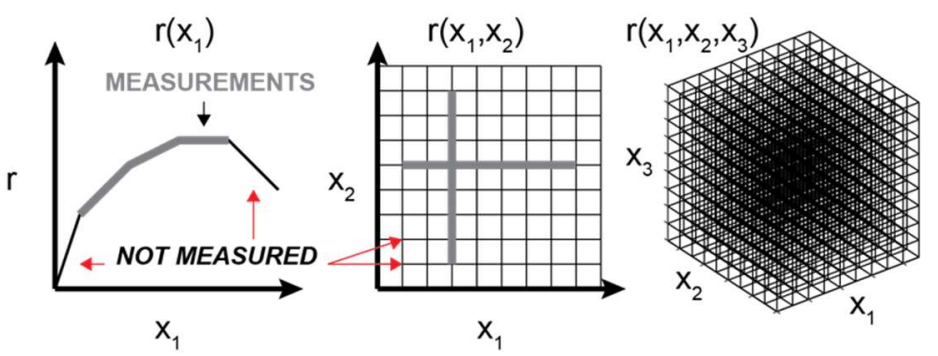

Fig. 2-2: The illustration of unrealistic musculoskeletal measurements for comprehensive model validation. About 9 points describing the moment arm profile per DOF (left plot) provide sufficient accuracy of approximations in our upper-limb model. The number of values increase as $9^{d}$, where $d$ is the number of DOFs increasing from 1 to 3 from left to right. The vertices in the grid demonstrate the required measurements, and gray lines illustrate the typical data measured experimentally. The typical hand muscle spans over 3 DOFs requiring more than 729 independent measurements (right panel).

\subsubsection{Validation Process}

We have identified three main challenges in creating a robust musculoskeletal model: i) errors in simulated muscle path for experimentally observed postures; ii) errors in simulated muscle path for experimentally unobserved postures; iii) force scaling problems between agonist-antagonist muscle groups. To mitigate these potential problems in model implementation, we used a threestep validation process where the muscle paths were validated iteratively in the first two steps (see below), and then their ensemble behavior was scaled in the third step.

\subsubsection{Step 1. Structural Validation for Experimentally Observed Postures}

We evaluated the anatomical validity of musculotendon paths relative to experimental measurements (see above, "Dataset 1. Moment Arm Measurements"), as shown in Fig. 2-3a. For each muscle, we initially adjusted the origin and insertion points using a standard 
anatomical reference (Netter, 2011). The musculotendon paths were repeated fine-tuned and checked to match the experimental and simulated moment arm values. This process involved adjusting the modeled muscle paths and comparing the new path to the published experimental data. The simulated relationships between moment arms and posture were computed using OpenSim data (See Dataset 3). These relationships were then compared to the experimental dataset to generate the quantitative metric of 'good enough' that corresponds to at least one of the following: 1) the root mean squared error (RMSE) value is less than $1 \mathrm{~mm}$ and the correlation coefficient $(\mathrm{R})$ is greater than 0.7 or 2 ) the RMSE value normalized to the difference of muscle lengths at the extremes of the full physiological ROM is less than 0.4 and the r-value is greater than 0.7 . The RMSE and $r$ values expressed the operational definition of acceptable quality within our study, but these values were chosen subjectively based on the examination of quality within the published model (Gritsenko et al., 2016; Saul et al., 2015a). These criteria ensured a low error between the simulated and experimental moment arm values. When these metrics were not met, indicating that muscle paths were incorrect, we interactively adjusted the muscle's geometric constraints in OpenSim. This time-consuming task required the understanding of multi-joint geometry of a muscle typically in 3 and up to 6 dimensions.

A simple example is shown in Fig. 2-3b, where the moment arm around MCP flex/ext DOF for the index finger extensor digitorum (ED2). The experimental data measured in human cadaver hands (Fowler et al., 2001) is closely represented by the simulated relationship after validation. To achieve this matched relationship, we moved a cylindrical wrapper slightly off from the center of rotation for this DOF, which caused the path to be accurate. While this type of adjustment is straight-forward for simple DOFs, this process becomes time-consuming and challenging for muscles with interactions between several DOFs, e.g., extensor pollicis longus. Many multidimensional muscle-DOF relationships have not been experimentally recorded and required additional error checks described in step 2. 

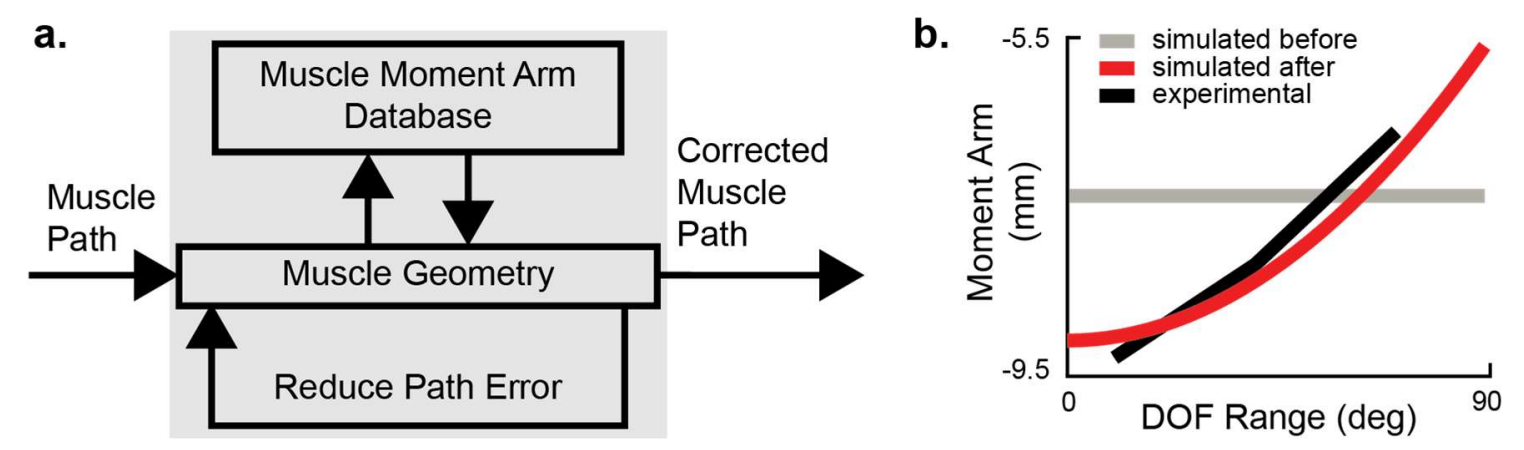

Fig. 2-3: The process of structural validation using an experimental dataset. (a) Flow chart diagram of moment arm fitting. The process of correcting moment arm values to create an accurate muscle path. (b) The fitting example of ED2 about the index MCP joint flex/ext DOF. The comparison of simulated (before = gray, after $=$ red) and experimental (black) moment arm profiles.

\subsubsection{Step 2. Structural Validation for Experimentally Unobserved Postures}

The goal of using biomechanical models for fundamental transformations of muscle activity into generated joint torques and valid limb motion requires data-driven model development. This task is challenging because experimental data for the description of moment arms in physiological postures is sparse. Moreover, the ability to subjectively examine muscle characteristics in the high-dimensional space $(d>3)$ of muscle postures is challenging. The following assisting analyses were devised to resolve potential model failures, i.e., structural and functional discontinuities, in the posture space without experimental measurements:

Zero-Crossing Error. This analysis identified the functional discontinuity corresponding to a flip in the direction of muscle torque, which was indicated by the zero-crossing event in its moment arm profile. This type of failure could arise from the unaccounted interactions in muscles spanning multiple DOFs. The testing for these crossing events was performed on each muscleDOF relationship in Dataset 1. Zero-crossings occur when the global maximum and minimum have opposite signs for one or more DOFs that the muscle spans. Fig. 2-4 shows an example with opposite signs of maximum and minimum values for extensor carpi ulnaris (ECU) in the original model. The superimposed profiles indicate changes in ECU wrist flexion-extension 
relationships as a function of 9 wrist pronation-supination postures. The validated model had the extrema with the same sign after ECU was corrected from slipping off the geometric wrapper in the extreme wrist extension. All muscles were tested, and all zero-crossing events found were then examined in OpenSim for potential structural errors.

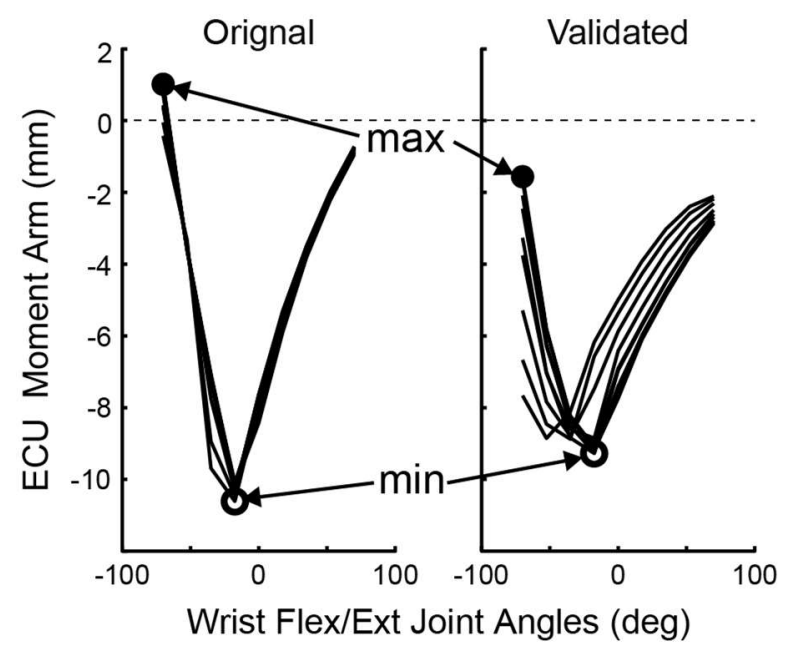

Fig. 2-4: Moment Arm Zero Crossing Tool. ECU wrist flex/ext relationship for the original (left) and validated (right) models. The ECU muscle spans wrist flex/ext and wrist pro/sup DOFs, and its moment arm value depends on both angles, which is evident from the multiple profiles for varying pro/sup angle. The presence of an unexpected zero-crossing from positive (flexion) to negative (extension) indicates a potential error at the joint extreme, which was corrected in the validated model.

Moment Arm Evaluation in Postural Extrema. The goal of this analysis was to review the profile of moment arms for agonist-antagonist muscle groups at the postural extremes, postures 0 , and $100 \%$ of full physiological ROM for each DOF. The postural extrema were the positions of interest because these locations often corresponded to failures in muscle wrapping geometry, causing opposite sign and profile errors in moment arms. For this reason, we plotted reports of moment arms at the extrema for each DOF for all agonist and antagonist muscle groups. Tentative failures were indicated by large differences (about 2 fold) in the magnitude of a single muscle or a small subset of muscles within one of the two groups. All suspected failures were examined in OpenSim for evidence of structural problems. 


\subsubsection{Step 3. Functional validation}

The necessity to collate data from multiple sources was expected to introduce an imbalance of measurements across multiple muscles, even though each muscle was consistent with a published observation (Goislard De Monsabert et al., 2018). To mitigate this problem in the holistic representation of muscle groups, we validated joint torque generation relative to the torque in maximum voluntary contractions (MVC) at specified postures (see above, Dataset 2 Torque Measurements). We simulated the torque measurements by locking the model in the experimental posture and supplying the maximum activation to all muscles with the same sign of moment arms around the DOF of interest. The force generation was posture dependent and determined by a Hill-type muscle model (Hill, 1938; Yakovenko et al., 2004; Zajac, 1989). The joint MVC torque was computed as the sum of all muscle cross-products between moment arms and forces, see Fig. 2-5. The scaling constant between experimental and simulated torques $\left(C_{F}\right)$ was used to adjust force generation across all muscles: $\tilde{F}_{\text {max }}=C_{F} * F_{\max }$. The starting $F_{\max }$ values were calculated as $F_{\max }=\sigma * P C S A$, where $\sigma$ is muscle specific tension with a value of 35.4 (Arkin, 1941; Brand et al., 1981) and PCSA is the physiological cross-section area (Chao et al., 1989). The maximum passive muscle force component was empirically set to be 10 percent of that muscles starting $F_{\max }$ value. All PCSA values originated from the same study (Chao et al., 1989) with the exception of biceps, which was missing from and measured from a separate study (Happee and Van der Helm, 1995). The adjusted $\tilde{F}_{\text {max }}$ values were computed from distal to proximal DOFs. This approach constrained solutions at proximal DOFs. For example, extensor digiti muscles span not only phalangeal joints, but also wrist joint. Their contribution to the wrist torque did not change when other muscles, e.g., extensor carpi ulnaris (ECU), were changed to match wrist MVC. 


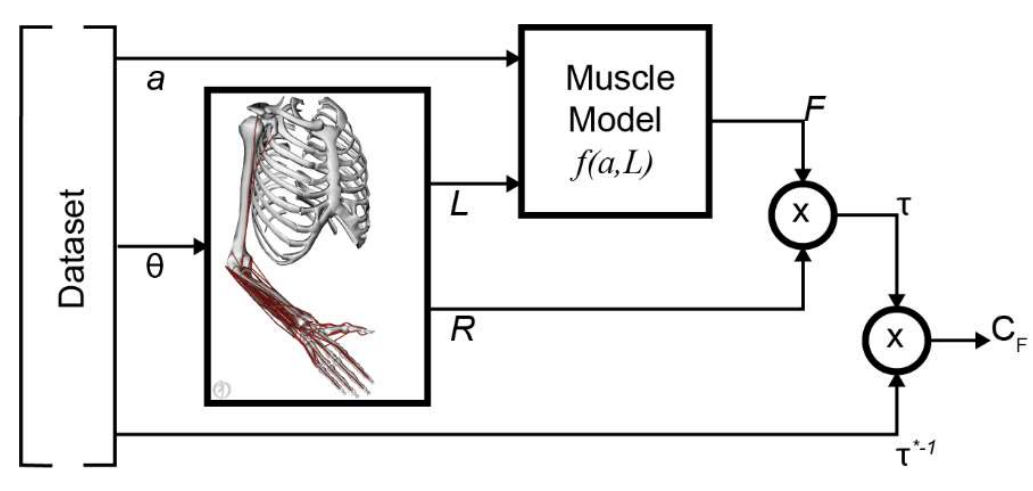

Fig. 2-5: Flowchart of scaling muscle force output. The experimental dataset of MVC measurements ( $\tau^{*}$ at $a=1$ ) depended on joint posture $(\theta)$ and were used to compute muscle force $(F)$ using a muscle model for isometric conditions. The muscle force $(F)$ was calculated as a function that depended on the muscle length $(L)$ and muscle activation (a). The scaling coefficient $\left(\mathrm{C}_{\mathrm{F}}\right)$ was computed using the simulated $(\tau)$ and experimental $\left(\tau^{*}\right)$ torques. The simulated torque was calculated by summing the muscle forces multiplied by the muscle moment arms $(R)$ about each DOF.

\subsubsection{Statistical Analyses}

The comparison of dynamic and static representations between moment arms and the corresponding ROM was performed using k-means clustering of the similarity index (Gritsenko et al., 2016). All statistical measures used a significance value of $\alpha=0.05$. The specific tension comparisons were performed by an ANOVA with a Tukey post hoc test to determine which groups were different.

\subsection{Results}

In this study, we developed a validation process for the structure and function of detailed musculoskeletal models for further implementation in real-time applications. We used a combination of quantitative and qualitative metrics to correct anatomical errors during the process of adjusting muscle paths relative to the skeletal landmarks to fit available experimental relationships. Then, we applied our quantitative analysis to identify two possible muscle path errors in the absence of experimental observations: $i$ ) the function change error due to the zerocrossing of muscle moment arm profiles, ii) the inadequate force generation in extreme 
postures. Each instance of tentative errors identified with these methods was examined and corrected, if necessary. These structural corrections were followed by the adjustment of force generation parameters for agonistic muscle groups validated by published MVC measurements. We examined the resulting model to test previous assumptions about the muscle-specific tension and evaluated the potential discrepancies of calculating torques with the assumption of constant or variable muscle moment arms at multiple wrist postures.

\subsubsection{Validation Process}

Fig. 2-6 shows the quantitative comparison of structural quality before and after muscle path validation. We assessed the errors in muscle paths for 33-musculotendon actuators controlling 18 DOFs of the arm and hand. Each path spans on average 3 to 4 DOFs with EPL spanning the maximum of 6 DOFs in this model, as shown in columns of original (left) and validated (right) panels with categorical representations. The 'good' category (green) in Fig. 2-6B was associated with the least amount of error between the experimental (Dataset 1) and simulated (Dataset 3) moment arm measurements. The categorical errors range from red the worst to green the best. The red category represents a sign flipping error, which causes inconsistencies in the calculation of torque. The pink, orange, and yellow categories are not as degrading to the model. The pink means that the profile error was incorrect and opposite what was desired. The orange represents an error where the profile was correct for some of the range of motion but incorrect elsewhere. The yellow represents the simulated profile shape matching the desired, but the scaling was incorrect.

The accepted range of regression $r$-values in the "good" category group was 0.72 and higher, with an average of $0.92 \pm 0.07$ (s.d.) for the muscles in that group. The original model contained only $8.6 \%$ of muscles that met the requirement to be in the 'good' category. After the validation process, $73.5 \%$ of all muscles were in the 'good' category. The error categories from severe (red) corresponding to the incorrect flip in the sign of moment arm to moderate (yellow) 
corresponding to the static offset in error were assigned to $26.5 \%$ of muscles (an improvement from $91.3 \%$ ). Overall the muscle paths have improved through the validation process, but the profile error was not corrected in 8 thumb and 5 wrist muscle paths. These high dimensional relationships were difficult to correct manually using the iterative process described in Step 1 of methods and may require an automated optimization in the future work. Not all of the muscles made it to the "good" category, but all of the muscles showed improvement due to the validation process. The overall improvement of model quality was assessed with a metric that assigns categorical scores to each color ( $r e d=1$, pink $=2$, orange $=3$, yellow $=4$, and green $=5$ ). The total scores were calculated only for muscles in both models and were 277 before and 418 after the validation with iterative adjustments, which is a 1.5 times improvement.
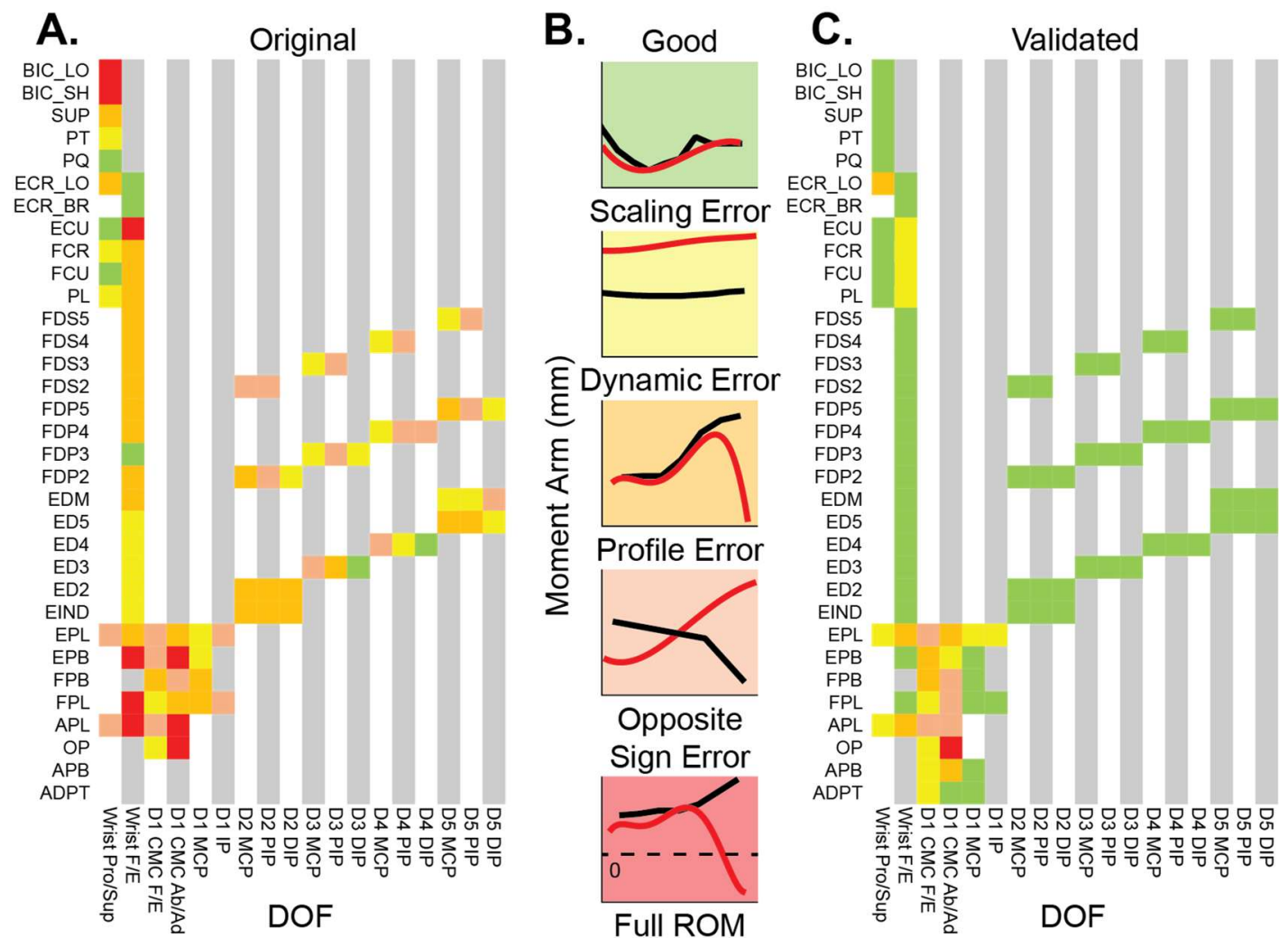

Fig. 2-6: The categorical comparison of structural quality between models with and without structural validation plotted as categories for all DoFs and all muscles (vertical labels). (A) The model from (Gritsenko et al., 2016; Saul et al., 2015a) before the manual path 
validation. The categorical values are shown for all muscle-DOF relationships. (B) The examples of all categories. (C) The same model after the manual path validation.

The experimental dataset sparsely represented all the muscle-DOF relationships. The unobserved domain was examined for possible zero-crossing errors. Fig. 2-7 shows the two distributions of all the muscle-DOF zero-crossing instances relative to the normalized posture within all possible multidimensional postures. Each instance of zero-crossing in Fig. 2-7A was examined and iteratively corrected if the crossing occurred due to problems with wrapping geometry. The distribution of zero-crossing instances had no strong preference relative to the ROM before and after the validation. The zero-crossing instances expected from the published experimental moment arm data were observed for two DOFs-wrist pronation-supination and CMC flexion-extension. The errors were removed in wrist sup-pro, wrist ext-flx, CMC ad-ab, and digit DOFs. Since the zero-crossing instances were occurring outside of the domain captured within the experimental dataset, the total number of these instances was bigger than the sign errors shown in Fig. 2-6A.

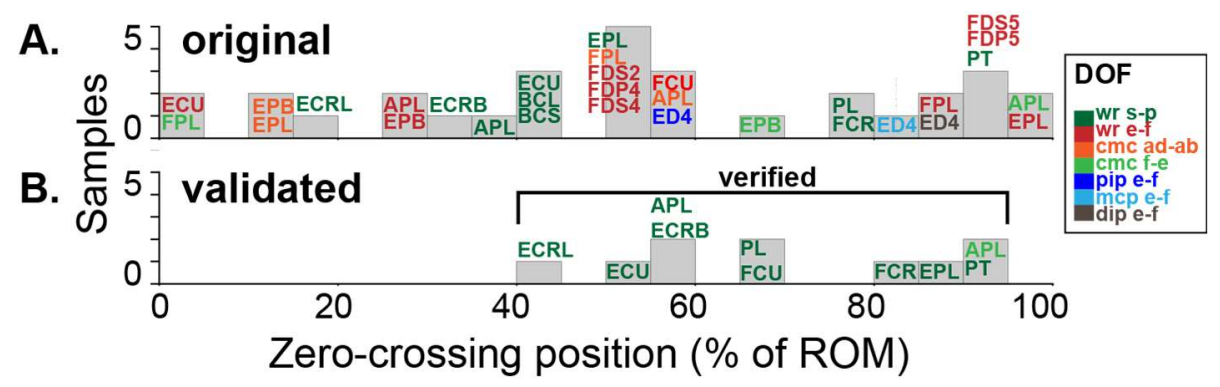

Fig. 2-7: Histograms of muscle moment arm zero-crossing events as a function of position. The colors represent the DOFs (described in the legend) where the crossing takes place. The distributions are shown for original (A) and validated (B) models.

Next, we scaled the model force generation using the MVC values (Dataset 2) for digit and wrist DOFs. The values for the $F_{\max }$ parameter in the Hill-type muscle model were adjusted in isometric simulations for specific postures corresponding to Dataset 2, as described in functional validation (see Step 3 in Methods). Before and after joint torques are shown in Fig. 2-8A. The initial maximum force values produced the most error at the wrist joint, which 
occurred due to the finger and wrist muscles contributing to the torque at the wrist joint. Our scaling process scales the finger muscles and then scales the wrist muscles to account for the force being produced by the finger muscles at the wrist. This process generates a physiologically relevant maximum torque at the wrist. The thumb muscles were not wholly corrected to match the desired toque, which occurred because we were never able to completely correct the thumb moment arms (shown in Fig. 2-6C) due to complex muscle functions at the thumb. After scaling the maximum force values to have the model produce physiological torque values, we wanted to show that the specific tension values for the muscles were still within the physiological range. We calculated the specific tension from the scaled model as $\sigma=F_{\text {max }}{ }^{\prime} / P C S A$, where PCSA is the physiological cross-section areas we used in step 3 of the validation. Fig. 2-8B shows our specific tension values compared to published experimental values calculated from cadavers and values calculated from 4 other musculoskeletal models. The calculations for the specific tensions shown for the 4 models were calculated by Buchanan in 1995 (Buchanan, 1995). We ran an ANOVA with a Tukey post hoc test that showed the specific tension values from the "other models" group in Fig. 2-8B is significantly higher $(p<0.05)$ than the other two groups. The specific tension values from the muscles in our model were not statistically different from the experimentally measured values. 

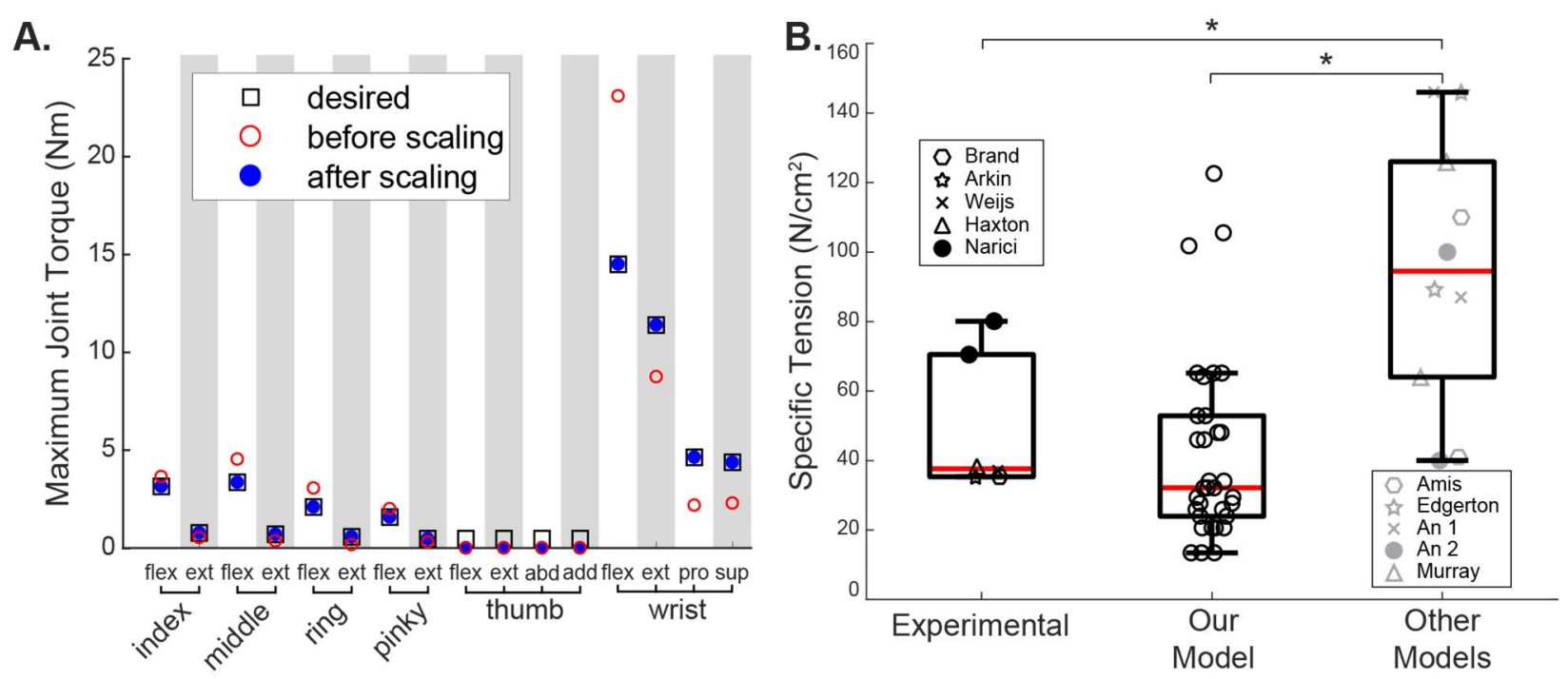

Fig. 2-8: Functional scaling process. (A) The torque profiles were reconstructed for eight-arm and hand DOFs. The panels represent two antagonistic actions for experimental (black) and model data. The black squares are the published maximum torque values (references for each value are in Table 2-2), the red circles are the torque values created from the updated muscle paths with a Hill-type muscle model, and the blue circles are the torque values after introducing the force scaler. (B) Muscle specific tension values. The experimental group came from published experiments to calculate the specific tension from cadaver measurements (Arkin, 1941; Brand et al., 1981; Haxton, 1944; Narici et al., 1988; Weijs and Hillen, 1985). Our Model group was the specific tension values from our musculoskeletal model after the maximum muscle force scaling. The other model group are specific tension values calculated in (Buchanan, 1995) for 4 musculoskeletal models (Amis et al., 1979; An et al., 1981; Edgerton et al., 1990; Murray et al., 1995) The asterisk marks that the Other models specific tension values are statistically significantly larger $(p<0.05)$ than the experimental or our model group.

\subsubsection{Constant vs. dynamical moment arms}

The use of constant relationships between moment arms and postures is a convenient approach in musculoskeletal modeling. However, is this constant relationship assumption an adequate approach, or must we spend additional time detailing accurate muscle paths for a full description of force generation? To address this, we examined the force generation capacity lost due to the assumption of a constant moment arm relationship. We tested the following predictions:

1) The dynamic muscle moment arms are smaller than the constant muscle moment arms when the muscle length is long. 
2) The dynamic muscle moment arms are larger than the constant muscle moment arms when the muscle length is short.

For example, flexor carpi radialis (FCR) wrist flexor moment arm increases with the increase in wrist flexion, shown in Fig. 2-9A. Similarly, the moment arm of wrist extensor carpi radialis longus $(E C R L)$ increases with the increase in wrist extension. The changes in torque generation in postural extrema would compensate for the length-force relationship and correspond to our predictions above. These general relationships were homologous across wrist flexors and extensors, as shown by the correlation analysis of moment arms in Fig. 2-9B\&C. We used the method previously developed in Gritsenko et al. (Gritsenko et al., 2016) to evaluate the similarity of mechanical muscle properties using a clustering analysis performed on the heterogeneous variance explained (HVE) metric computed from the coefficient of determination $\left(R^{2}\right)$ for the moment arm relationships. The HVE was computed as $1-R^{2}$ for $R<0$ and $1+R^{2}$ for $R \geq 0$. This metric identifies agonist muscle pairs by short HVE distances close to 0 and antagonist pairs close to 2. This analysis in Fig. 2-9B showed apparent similarities in moment arm relationships among flexors and extensors with separation of the flexors and extensors (HVE $\approx 1.6)$. The correlation matrix is shown in Fig. 2-9C further supports this description with highly correlated flexor moment arms (see lower right quarter in Fig. 2-9C) and most of the extensors (see upper left quarter in Fig. 2-9C). Flexor and extensor moment arm profiles around wrist flexor-extensor DOF are highly antagonistic, as shown with the remaining two quarters in Fig. 2-9C. Next, we tested the differences of moment arm averages in extreme postural quartiles (dynamic condition, the solid line in Fig. 2-9A) against the averages across the full ROM (constant conditions, dashed line in Fig. 2-9A). We found that the dynamic moment arms are significantly smaller than the static moment arms by $15.0 \pm 14.8 \%$ when the muscles are long and vice versa by $19.9 \pm 14.2 \%$ when the muscles are short, both comparisons having $p$-values $<0.0001$. Since 
moment arms scale torque, this difference constitutes a $34.9 \%$ error in simulated wrist flexorextensor torque generation in extensive movements.

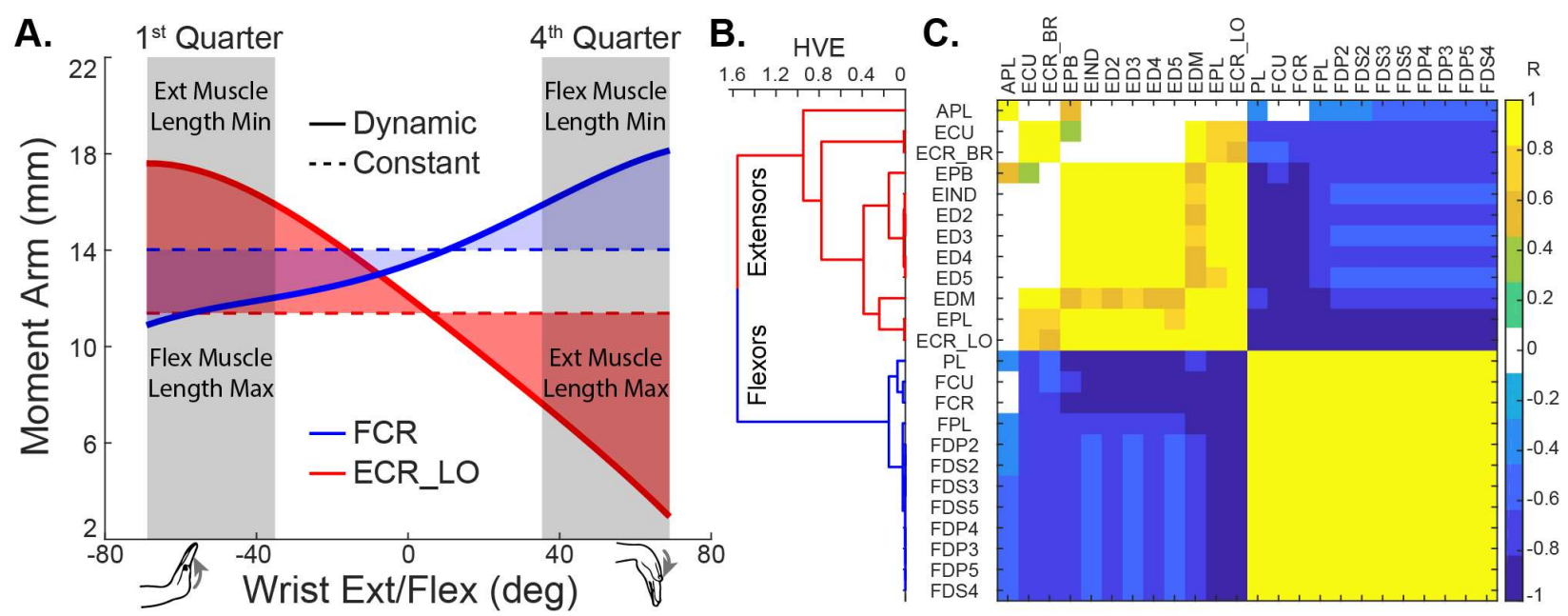

Fig. 2-9: The comparison of static and dynamics moment arms. (A) Example wrist flexor (blue) and extensor (red) moment arm profiles around wrist extensor-flexor DOF. (B) The cluster analysis of HVE metric of profile similarities. (C) The correlation matrix of r-values between pairs of muscle moment arm profiles.

\subsection{Discussion}

In this study, we developed a novel validation process that improves the anatomical and functional accuracy of musculoskeletal models. In this process, we first analyzed muscle path accuracy by comparing simulated muscle moment arms against our measured moment arm dataset (See Datasets) under different postures to correct the simulated muscle paths. The second step took the corrected muscle moment arm values and created torques with a Hill-type muscle model (Zajac, 1989) that were then scaled to match the maximum torques recorded in the literature (See datasets). Scaling the forces was vital because it adjusted the torque values of the model to stay within the biological boundaries of torque output. With the validation process complete, we have developed a biologically accurate musculoskeletal model and moved to focus on addressing its applications and how it can be used to resolve critical questions about musculoskeletal driven prostheses. The question we wanted to answer was, 
"What do we lose when we assume constant moment arms instead of their true dynamic nature for the wrist flexion-extension DOF?"

We found that the constant moment arm assumption is more accurate to physiological measurements in the middle of the ROM for wrist flex/ext but becomes more inaccurate as you move toward the extremes. The dynamic moment arm profiles of wrist flex/ext near the extremes (Fig. 2-7A grey shaded regions) varied, on average, approximately $17 \pm 14 \%$ from the constant moment arm values. For example, this would require a prosthetic user to exert more effort to control a device using constant moment arms because they must account for these differences, and they are not benefiting from the dynamic relationships observed between muscles during movement. This means that to maintain a flexed wrist, they need to apply more flexion activation than natural to keep the wrist flexed and the more wrist extension activation than natural to keep the wrist extended. Gonzalez et al. 1997 showed that the maximum force production from a joint was dependent on posture, which would mean that a constant moment arm approach would not describe this relationship. Even though the constant moment arm approach has the drawback of miscalculating the torque generation, it has the benefit of simplifying complex muscle-DOF relationships. Conversely, dynamic moment arms are more biologically accurate than constant moment arms but should not be used unless the muscle DOF relationships are checked with a validation process like ours. Without being validated, the dynamic moment arms could be worse than the constant moment arm assumption and cause inconsistencies if undesired zero-crossings occur.

The first step of the validation process we described in this study takes the available moment arm data from literature and uses it to validate our model for the full physiological ROM. Many models are only validated for a specific task and ROM, and it can cause problems when others try to use the model for tasks and ROMs outside the validated states. We required a large dataset on muscle-DOFs to validate the model, but there was sparse data to be found in the 
literature on these relationships. The data available was measured from people of different ages and genders, which could cause inconsistencies in the dataset. Moreover, the moment arms available in literature only contained values for a subset of possible postures. This issue is due to it being nearly impossible to measure all possible postures for all the different DOFs. To addressed the sparse dataset issue, we developed evaluation tools to look at the moment arms in all possible postures find anomalies to help us maintain correct moment arms. These anomalies tended to occur at the extremes of ROM due to the model moving toward a sharp angle between bones and the muscle path moving incorrectly in that specific posture. The example shown in Fig. 2-4 had this happen to the ECU muscle during extension. This can cause inconsistencies in the model if the sign flipping is not meant to happen. Fig. 2-7 shows 31 zero crossings before and the 10 expected zero crossings after the validation process. By using the evaluation tools, we were able to keep the muscle-DOFs consistent through the ROM. Fig. 2-6 is the qualitative evaluation, which shows that the model was improved anatomically during the first step of the validation process. The moment arms about the thumb have the most issues and are due to the complex interactions between the moment arms about the CMC joint, making them more challenging to correct. However, the validation process successfully identified the errors at this DOF, and future work will be to correct them. Issues such as these in the anatomical structure, as well as using lumped parameters in the muscle model, require the second step of the validation process to ensure the model's function performance.

Our functional validation process ensures that the torque output from the model does not exceed physiological values. Otherwise, any inferences made from the model about the biology would be invalid. We perform this process by scaling the model's simulated maximum torque to keep it within limits set in the literature (see torque dataset). The torque scaling was necessary due to two main issues: the first was due to inconsistencies in the measured moment arm dataset, and the second one was due to inaccuracies in the muscle model from our lumped 
maximum force simplification. The muscle model inaccuracies stem from having an incorrect maximum force estimate and not accounting for the muscle pennation angle. The incorrect maximum force is due to there being many estimated values published in the literature, which happens due to it being somewhat subject-specific. The pennation angle was ignored as an assumption that the fiber angle in the case of the forearm muscles did not contribute to a substantial change in the force, and it was done to simplify the muscle model. After we completed the scaling, which provided new lumped maximum force parameters, we wanted to check to see if the new values were reasonable. To do this, we calculated the specific tension of the muscles using published PCSA values (Chao et al., 1989; Happee and Van der Helm, 1995) and found that the specific tension was not significantly different from the published experimental values (see Fig. 2-8). These results further support that our model is physiologically accurate. However, specific tension values calculated from 4 other musculoskeletal models in 1995 by Buchanan (Buchanan, 1995) are significantly higher than ours (see Fig. 2-8 for model references). The high specific tension values for those models could indicate a need for a functional validation to scale the muscle forces to ensure they are physiological. Our model is ideally representative of the general population due to all the datasets used to validate it were combined across studies based on the general population.

We have developed a generic arm model that could be used for prosthetic control or the biomechanical analysis of movement. The model was designed to represent an average young adult by having its torque output and muscle paths match the published average data. Future development will test if the model accurately represents the general population or if it requires subject-specific scaling. If a generic model can adequately describe the musculoskeletal dynamics of this broader population, it would greatly simplify its implementation in many applications requiring no user-specific adjustments. The data from our model can even be accessed in real-time (Sobinov et al., 2019) applications, which provides a valuable resource for 
human-machine interfaces. An example would be to monitor an individual's health or control a prosthesis using live approximated muscle properties, such as length and joint moment arms. Our future directions are to expand our model to include the proximal arm muscles as well as a leg and trunk model to move toward a full-body model that is validated for the entire physiological ROM.

There are several limitations to the validation process, most relating to the sparse dataset used to compare simulated and published data. Hand and arm muscles span on average 3 DOFs, but often data is only published for one DOF with the other DOFs placed in a standard posture and never moved. This led us to create validation datasets that were combined from available literature and contained variances due to inter-subject variability and measurement techniques (e.g., cadaver or magnetic resonance imaging). These variances have the potential to cause inconsistencies in the muscle parameters (See Datasets). We combated these inconsistencies with the validation tools we created to search for inconsistencies in the muscle path. Another limitation is that the muscles are modeled having muscle fibers with no pennation angle as well as a stiff tendon assumption, which could cause minor errors in the force production. We compensated for some of these errors in the muscle torque scaling described in the validation step two. Despite these limitations, the validation process still works to highlight errors in the musculoskeletal model for the user to correct.

\subsection{Conclusions}

In conclusion, we developed a validation process to help create a robust and physiologically accurate model. We also found that small changes in moment arm profiles can amount to substantial differences in the torque production at a joint. These results came from our validation process, looking at both the physical structure of the model as well as the functional torque output. It is essential to check both because it ensures that the muscle model properties, 
as well as the muscle paths, are accurate and within physiological limits. This process makes a more accurate model than one that only checks the muscle moment arms. Our approach, or one similar, will benefit anyone working to create a physiologically accurate model.

\subsection{Supplementary Material}

The supplementary materials were submitted with the dissertation. It includes PDFs of all the muscle-DOF profiles from experimental data and simulated that was used to created Fig. 2-6. It also includes an Excel file for each muscle-DOF describing the experimental data profile and its source. 


\section{Chapter 3 Prosthetic Controller Approach Using A Refined}

\section{Musculoskeletal Model}

(this chapter is in preparation to be submitted as "Boots M, Sobinov A, Gritsenko V, Gaunt R, et al., Yakovenko S (2019) A Prosthetic Controller Approach Using A Refined Musculoskeletal Model')

\subsection{Introduction}

Advanced complex limb protheses and exoskeletons are becoming tools that can restore lost functionality or augment natural abilities. New prosthetic hardware is expanding the set of functional movements that prostheses can make, allowing the movements to become similar to those of an intact human arm (Carrozza et al., 2006; Kyberd et al., 2001; Resnik et al., 2018a). While these sophisticated machines should have a high adoption rate, amputees are often choosing a passive prosthesis or none at all. Around 10 to $25 \%$ of upper limb amputees do not use a prosthesis (Berke et al., 2010; Biddiss and Chau, 2007; Pinzur et al., 1994; Raichle et al., 2008). Of the remaining upper limb amputees (75 to $90 \%$ ), only half choose a powered prosthesis (Whiteside et al., 2000). The main reason for their choice is associated with the frustration of the powered prosthesis performing actions slowly or incorrectly, as well as how easily the prosthesis can break. The current prostheses that are controlled by muscle activity lack robustness (Cordella et al., 2016), and with the number of upper limb amputees expected to at least double from 2005 to 2050 (Ziegler-Graham et al., 2008), there is a need for developing better and more robust prosthetic controllers.

Myoelectric prostheses are powered prostheses that use electromyography (EMG) (i.e., measurements of electrical activity in response to nerve stimulation in a muscle) as the control input from the user. The clinically used control approach for a myoelectric prosthesis is direct 
control, which links an agonist-antagonist muscle pair to move a prosthetic DOF (Ison and Artemiadis, 2014). This approach achieves satisfactory control for a single DOF (e.g., open and close a gripper), but controlling multiple DOFs is associated with a cognitive load (Cordella et al., 2016). The reason for the cognitive load is due to the amputee needing to use a switching technique to change the currently controlled DOF to another DOF. Muscle co-contraction, which is activating antagonististic muscles at the same time, above a threshold is a method commonly employed to operate DOFs sequentially (Schultz and Kuiken, 2011). Pressing on a force sensor or mechanical switch are other methods to switch between the current DOF being controlled (Schultz and Kuiken, 2011).

There have been attempts to create more intuitive methods for prosthetic control where the user's intent is extracted from the EMG. The most common method is based on the direct transformation from EMG to movement through pattern recognition (Birdwell et al., 2015; Graupe et al., 1977; Scheme et al., 2014; Scheme and Englehart, 2011; Smith et al., 2014). The goal is to create a classifier that can associate EMG activity with a specific movement (e.g., wrist supination, wrist pronation, hand grasp, hand open, etc.). This process requires the collection of training data for each movement, and the quality of the transformation deteriorates with time requiring periodic recalibration. When the process works, amputees can move their prosthesis intuitively without a cognitive load, but there are two main drawbacks to using pattern recognition. The first is it requires a large amount of training data to achieve reasonable control. The second is how the controller has reduced functionality in limb postures that were not trained. The pattern recognition approach has potential, but it has been shown to not always be more intuitive and straightforward for the user than the direct control method (Resnik et al., $2018 b)$. One of the main issues with decoding the movement intent is that there is a nonlinear relationship between the EMG signals and the movement (Dantas et al., 2019). The use of a 
biomimetic approach (i.e., modeling biological processes) could potentially simplify the process of decoding motor intent.

A biomimetic approach to interpret motor intent is to use muscle models and musculoskeletal dynamics to translate EMG into its intended movement. The goal is to make the prosthetic control intuitive by having a person perform a movement and decipher the intended movement through a musculoskeletal model in real-time. The approach uses recorded muscle activity as an input and processes it through biomechanics to generate muscle forces. The most commonly used muscle model is a Hill-type muscle model (Hill, 1938; Romero and Alonso, 2016; Winters and Stark, 1987; Yakovenko et al., 2004; Zajac, 1989), and it is described by Equation 3-1 to 34. The cross product of forces and moment arms for each DOF are summed to represent the joint torque. Torques are used in the equations of motion to simulate the limb movement. This process accounts for some of the non-linearities in the EMG-to-motion transformation by modeling the intrinsic muscle dynamics and its interactions with body mechanics that cause non-linearities. The approach has been used as the principle for prosthetic control (D. Crouch and Huang, 2016a; Sartori et al., 2018). This method has simultaneously controlled 2 DOFs with constant moment arms, where hand grasping was controlled by antagonistic finger muscles, and wrist flexion/extension was controlled by antagonistic wrist muscles (Crouch et al., 2018; Pan et al., 2018a). This method has the capability to control multiple DOFs (two or more) simultaneously and could complement new, more complex prosthesis designs.

In this study, we created the computational framework for real-time EMG-to-motion transformations using the description of arm and hand biomechanics. The spatiotemporal sequence of EMG bursts can be approximated by a set of Gaussian functions. This approximation captures complex intralimb patterns of activity (Yakovenko et al., 2011) with a reduced set of parameters that can be optimized to generate the desired movement. Thus, we investigated offline if Gaussians could be used to drive whole hand movements. The concept of 
using a reduced set of parameters for movement control is related to the dimensionality reduction approaches (Ivanenko et al., 2004; Lambert-Shirzad and Van der Loos, 2017; Popov and Yakovenko, 2015; Shaharudin et al., 2014). This concept originates from the idea that the central nervous system relies on the hierarchical organization with a small set of controlled parameters at a high-level (Churchland et al., 2012). In this study, we attempt to identify the essential low-dimensional structure required for the control of the arm and hand. From this structure, we developed a group signal processing method to estimate the signals from unrecorded muscles, which provided a method to decipher movement intent with minimal control signals. We used this movement intent to simulate hand movements in real-time as well as control prostheses, see supplementary materials for videos.

\subsection{Methods}

\subsubsection{Dynamic Control of the Musculoskeletal Model}

Our control approach was to decode the intent from forearm muscle activity by accurately modeling the transformation of muscle recruitment signals into musculoskeletal dynamics, which can then be used to control a prosthesis. Fig. 3-1 shows the corresponding control-flow diagram of our approach, with EMG inputs and prosthetic control as outputs.

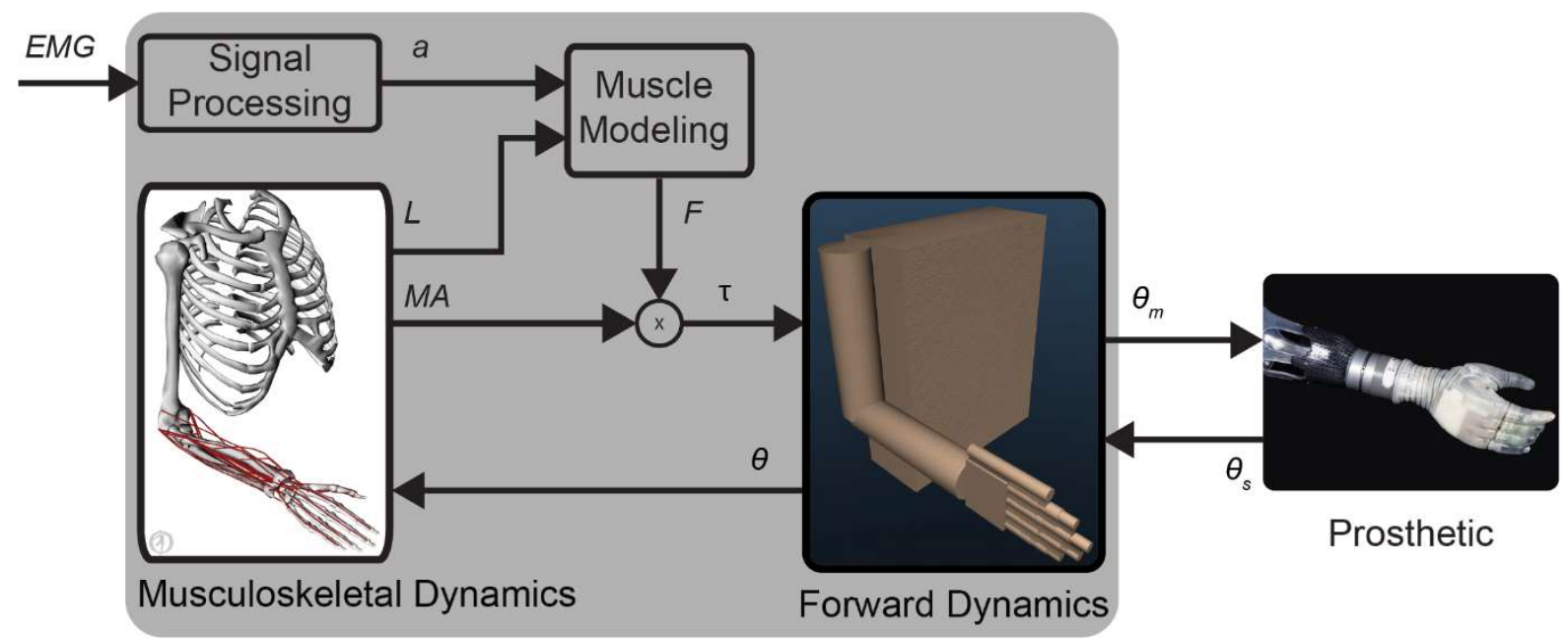


Fig. 3-1 Prosthetic Hand Biomimetic Controller. Control flow diagram for biomimetic myoelectric control of a prosthetic. The EMG recordings are inputted into the biomimetic controller (shaded region) and are used to produce joint torques that move an arm and hand model hand segments in real-time forward dynamic simulations. The simulated joint angles $(\theta)$ can be mapped $\left(\theta_{m}\right)$ to move a prosthetic hand. The prosthetic can then provide feedback $\left(\theta_{s}\right)$ to the controller to update the controller state.

We converted raw EMG to muscle activation (a) through the following process. First, the signal was demeaned and then full-wave rectified. The resulting signal was then thresholded to remove low-level noise that was estimated at rest as the average of the signal plus 6 standard deviations. EMG signals were normalized using their corresponding maximal values recorded within a set of 10 standard movements, assuming that this level corresponded to $15 \%$ of their maximal recruitment. The normalized signals were low-passed filtered using a $200 \mathrm{~ms}$ moving window average. Additional optimizations of recruitment estimates were required to reduce kinematic errors in online tasks.

Muscle contraction generates a pulling force around the DOFs the muscle spans. The force magnitude is described by the Hill-type muscle model (Gillard et al., 2000; Hill, 1938; Yakovenko et al., 2004; Zajac, 1989), shown in Equation 3-1 to Equation 3-4.

\section{Equation 3-1}

$$
F(a, L, \dot{L})=a \cdot F_{\text {max }} \cdot F_{L}\left(L_{\text {norm }}\right) \cdot F_{V}(\dot{L})+F_{\text {pass }} \cdot F_{P}(L)
$$

where muscle force $(F)$ is a function that depends on the muscle activation level $(a)$, the musculotendon length $(L)$, and velocity $(\dot{L})$. The musculotendon length is posture dependent. The normalized muscle length $\left(L_{\text {norm }}\right)$ was scaled to the muscle's maximum and minimum length $\left(L_{\text {norm }}=\frac{L-L_{\min }}{L_{\max }-L_{\min }} \in[0,1]\right)$, where $L_{\max }$ is the total maximum muscle length and $L_{\min }$ is the minimum muscle length. The evaluation of maximum force for each muscle $\left(F_{\max }\right)$ is described in Chapter 2 and is listed in Supplementary Table 3-2 along with the maximum passive force parameter $\left(F_{\text {pass }}\right) . F_{L}$ (described in Equation 3-2) is the force-length relationship, 
$F_{V}$ is the force-velocity relationship (described in Equation 3-3), and $F_{P}$ is the dependence of passive force on musculotendon length (described in Equation 3-4).

\section{Equation 3-2}

$$
F_{L}\left(L_{n o r m}\right)=2.5 \cdot L_{\text {norm }}-1.25 \cdot L_{\text {norm }}^{2}
$$

The equation was a compromise that attempted both to represent the short-stiffness effect on the force-length relationship (Gillard et al., 2000) and to reduce the overestimation of force when a constant slope was assumed (Yakovenko et al., 2004).

\section{Equation 3-3}

$$
F_{V}(\dot{L})=\left\{\begin{array}{c}
1+\left(\frac{1-\exp \left(-0.425 \cdot \dot{L} / L_{o}\right)}{1+\exp \left(-0.425 \cdot \dot{L} / L_{o}\right)}\right), \dot{L} \leq 0 \\
1+0.8\left(\frac{1-\exp \left(-0.425 \cdot \dot{L} / L_{o}\right)}{1+\exp \left(-0.425 \cdot \dot{L} / L_{o}\right)}\right), \dot{L}>0
\end{array}\right.
$$

where the rest length of the muscle $\left(L_{R}\right)$ is assumed to be half of the muscles range of motion $\left(L_{o}=\left(L_{\max }+L_{\min }\right) / 2\right)$.

\section{Equation 3-4}

$$
F_{P}(L)=\left\{\begin{array}{c}
\frac{\exp \left(2 \cdot \frac{L-L_{\text {pass }}}{L_{\max }-L_{\min }}\right)-1}{\exp (1)-1}, L>L_{\text {pass }}, \\
0, L \leq L_{\text {pass }}
\end{array}\right.
$$

where $L_{\text {pass }}$ is the slack length of passive tissue, indicating the onset of the passive force contribution. The magnitude of muscle torque $(\tau)$ is a cross-product of muscle moment arm $(\mu)$ and force $(F)$, shown in Equation 3-5.

\section{Equation 3-5}

$$
\tau(\mu, L, \dot{L}, t)=\mu \times F(a, L, \dot{L}),
$$

The muscle moment arms and lengths are posture dependent. Supplementary Table 3-1 and Table 3-2 describe all the simulated DOFs and muscle parameters. Rather than simulating full 
sliding kinematics, which can be computationally intensive and time-consuming, we used accurate approximations computed in real-time (less than $10 \mu \mathrm{s})$. These polynomial approximations (Sobinov et al., 2019) allowed us to model each muscle length and moment arm as functions of joint angles.

The muscle torques were then applied in the equations of motion using the MuJoCo physics engine (Kumar and Todorov, 2015; Todorov et al., 2012) to produce joint kinematics. The mechanical model implemented in MuJoCo was represented a human arm and hand. The mass of each segment was calculated assuming uniform density and dividing the hand volume into the segments of interest (Clauser et al., 1969). The joint angles (JA) calculated by the physics engine were used to update muscle state using polynomial approximations. When connected to a prosthetic hand, the joint angles were used as control signals to move the prosthesis together with the simulated hand. This process allows us to control any prosthesis with reasonable anthropomorphic correspondence between physiological and prosthetic DOFs. The implementation requires only linking the DOFs of the prosthesis to the DOFs of the simulated hand. The state of the prosthesis can also provide feedback to update the state in the physics engine to minimize the kinematic errors between the prosthesis and the simulated hand.

Our signal processing and model operated in real-time (less than 2ms loop latency), allowing the data from a participant to be used, and the current movement intent deciphered as quickly as possible. The short loop latency reduced numerical errors in the calculation of the muscle forces. Fig. 3-2 shows that the average iteration of the entire controller is less than $2 \mathrm{~ms}$. 


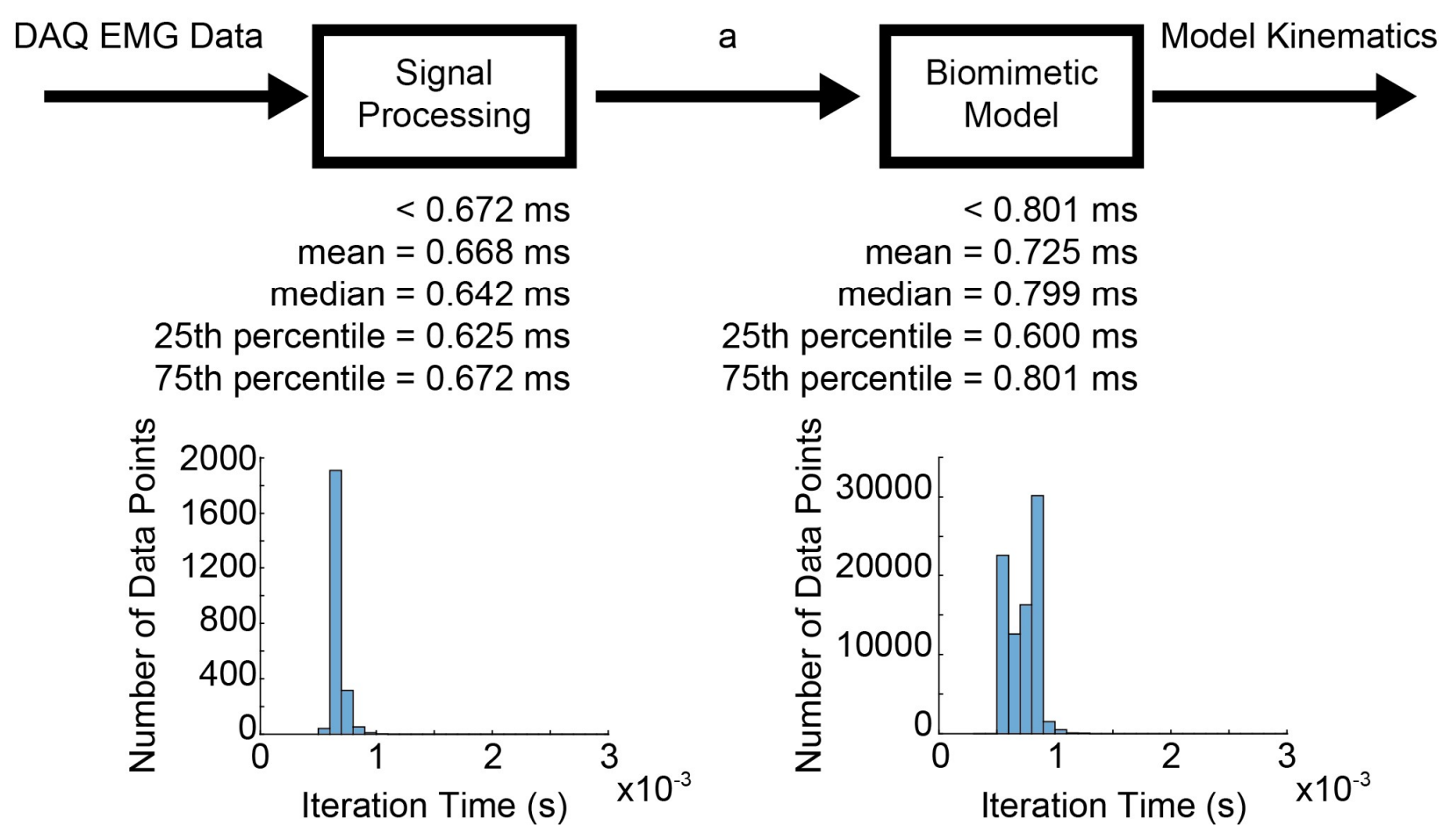

Fig. 3-2 Controller Loop Time. The controller consists of two parts signal processing with muscle state simulation and physics engine. Overall, the controller iteration time, on average, was less than $1.47 \mathrm{~ms}$.

\subsubsection{Data Collection}

For this study, EMG data were collected from two participants with and without transradial amputation for 10 movements described in Supplementary Table 3-3. EMG signals and arm and hand kinematics were collected at the University of Pittsburgh, and all procedures used in the study were approved by the University of Pittsburgh IRB. The movements were chosen to cover the entire ROM for all the DOFs and test the capabilities of the controller by actuating or maintaining the DOFs with varying duration and speed. Supplementary Table 3-1 and Table 3-2 provide more information about the muscles that are in the model and the DOFs that those muscles span. The muscles recorded in the healthy participant with intramuscular EMG were SUP, PTER, ECR_LO, APL, ED2, ED3, ED4, EIND, EPL, FPL, FDP4, FDP2, FCR, FDS3, FDS2, and FCU and the muscles recorded with surface EMG were BRR, BIC_SH, BIC_LO, TRI_LAT, TRI_LO, DELT_A, DELT_P, PECM_M, TERMA, APB. The muscles recorded in the 
amputee with intramuscular EMG were SUP, ED2, ED5, EPL, ECU, ECR_LO, PTER, FCR, FCU, FDS2, FDS4, and FDP3 and there were no surface EMG recordings. The intramuscular EMG sensors (Motion Lab Systems, Baton Rouge, USA) were placed with the guidance of ultrasound. Once the sensors were placed, the participants were asked to perform specific movements, while researchers tested signal quality and confirmed the muscles being recorded were accurate. After the EMG sensor validation was complete, the arm was wrapped in gauze to secure the EMG sensors. The healthy participant wore a glove that was instrumented with kinematic sensors (The Motion Monitor, Chicago, USA) to record hand kinematics.

\subsubsection{Optimization of Single Gaussian Approximations of EMG Signals}

When observing processed EMG signals, we noticed that their profiles appeared to be similar to Gaussian distributions. Thus, we wanted to see if there was potential to simplify the processed EMG signals to Gaussian signals for control. We used the recorded kinematics to create a Gaussian signal for each muscle that would reproduce each movement.

A Gaussian for each muscle was created with a multivariable optimization that aimed to minimize the error between the simulated and recorded kinematics. The optimized parameters were the mean (timing), standard deviation (width), and amplitude (height) of the Gaussian. The optimization was implemented with fmincon in Matlab (MathWorks version R2019a), which attempted to minimize the cost function, described in Equation 3-6. The optimization's initial conditions were based on the recorded EMG signals. The Gaussians for muscles not recorded were given the same initial conditions as muscles that performed the same action and were recorded. For example, if there was data recorded for FDP2 but not FDS2, then the initial conditions for FDP2 would be provided to FDS2. Each optimization iteration used the created Gaussians to simulate the entire movement and then compare the simulated with recorded kinematics. Once an optimization stopped, the parameters that produced the best kinematic result were empirically tuned further by hand, which resulted in slight adjustments to help 
produce a more accurate movement, and another optimization was performed. The reason this empirical tuning was necessary was due to the optimization getting stuck in a local minimum. The overall stop criteria, if reached, was when the kinematic root mean squared error (RMSE) for the 8 DOFs of interest (wrist pro/sup wrist flex/ext, thumb abd/add, thumb flex/ext, index MCP flex/ext, middle MCP flex/ext, ring MCP flex/ext, and pinky MCP flex/ext) was less than 0.4 radians. This value was chosen empirically to allow for some discrepancy between the instructional movement and the simulated movement, while still maintaining the simulated movement to be appropriately recognized as the desired movement.

\section{Equation 3-6}

$$
C=\frac{1}{n} \sum_{i=1}^{n} r m s\left(\theta^{*}-\theta\right)_{i}+0.5 \sum_{i=1}^{n}\left(1-R^{2}{ }_{i}\right)+\frac{0.01}{m} \sum_{i=1}^{m} a_{G_{i}}
$$

where $C$ is the cost value that was used by the minimization function, $n$ is the number of DOFs of interest, $r m s()$ is the taking the root mean squared, $\theta^{*}$ is the desired kinematics for the movement, $\theta$ is the simulated kinematics for the movement, $R^{2}$ is the coefficient of determinant between the desired and simulated kinematics, $\mathrm{m}$ is the number of muscles, and $a_{G}$ is the amplitude of the Gaussian.

Every movement required the optimization of the Gaussian mean, standard deviation, and amplitude, which resulted in optimizing 99 parameters. We developed an approach to reduce the number of parameters that needed optimized at once. We would optimize the muscles for distal joints and would drive proximal joints with kinematics. After we had all the muscles optimized for distal joints, we would then move to optimize the muscles for the proximal joints. Even though most muscles span multiple joints, we optimized the muscles for their primary function joint. For example, the index finger flexion/extension muscles are optimized to move the index finger and not the wrist, even though these muscles will contribute to the torque at the 
wrist. This method ensures that the muscles that move proximal joints can overcome the muscles that cross not only this joint but also the more distal joint. We perform separate optimizations on the muscles that drive each finger and thumb. As a result, each optimization required only 18 parameters. After the digit optimizations were done, the optimizations of the wrist DOFs starting with flexion/extension and finishing with pronation/supination were performed.

Once we generated Gaussian signals for each movement, we wanted to compare them to recorded and processed EMG to see if there was any structural similarity during muscle activation. We compared the signals by creating hierarchical clusters and tested for similarity with the Fowlkes-Mallow similarity index (Fowlkes and Mallows, 1983), shown in Results 3.4.2.

\subsubsection{Muscle Grouping Signal Processing}

Not all muscles can be recorded, which is mainly due to space constraints in getting the sensors placed. To overcome this issue, we designed an approach to provide muscles not recorded with signals based on their function and the other recorded muscles. The muscles were grouped into 5 levels, where each subsequent level shared a standard function. The first three levels are based on muscle function, and the last two are to provide a hand open-close function if only two muscles could be recorded. These groups allowed us to get data for muscles that were not recorded from muscles that performed similar actions. If there are multiple muscles with a recorded signal in the same group, the recorded signals are averaged together and used for other muscles in the group. Fig. 3-3 shows how the muscle groups and levels are divided with an example of this process shown by the colors. Seven of the muscles on the left have recorded data, and their corresponding color shows how that muscle's data is supplied to other muscles at different levels. The color changes when two signals have been averaged together to create a new signal that is supplied to other muscles in the group. This approach works well with single 
DOF joints, but it becomes difficult if a muscle's function does not have a single action. This can be seen in the thumb muscles, where some of them do not have many levels of grouping.

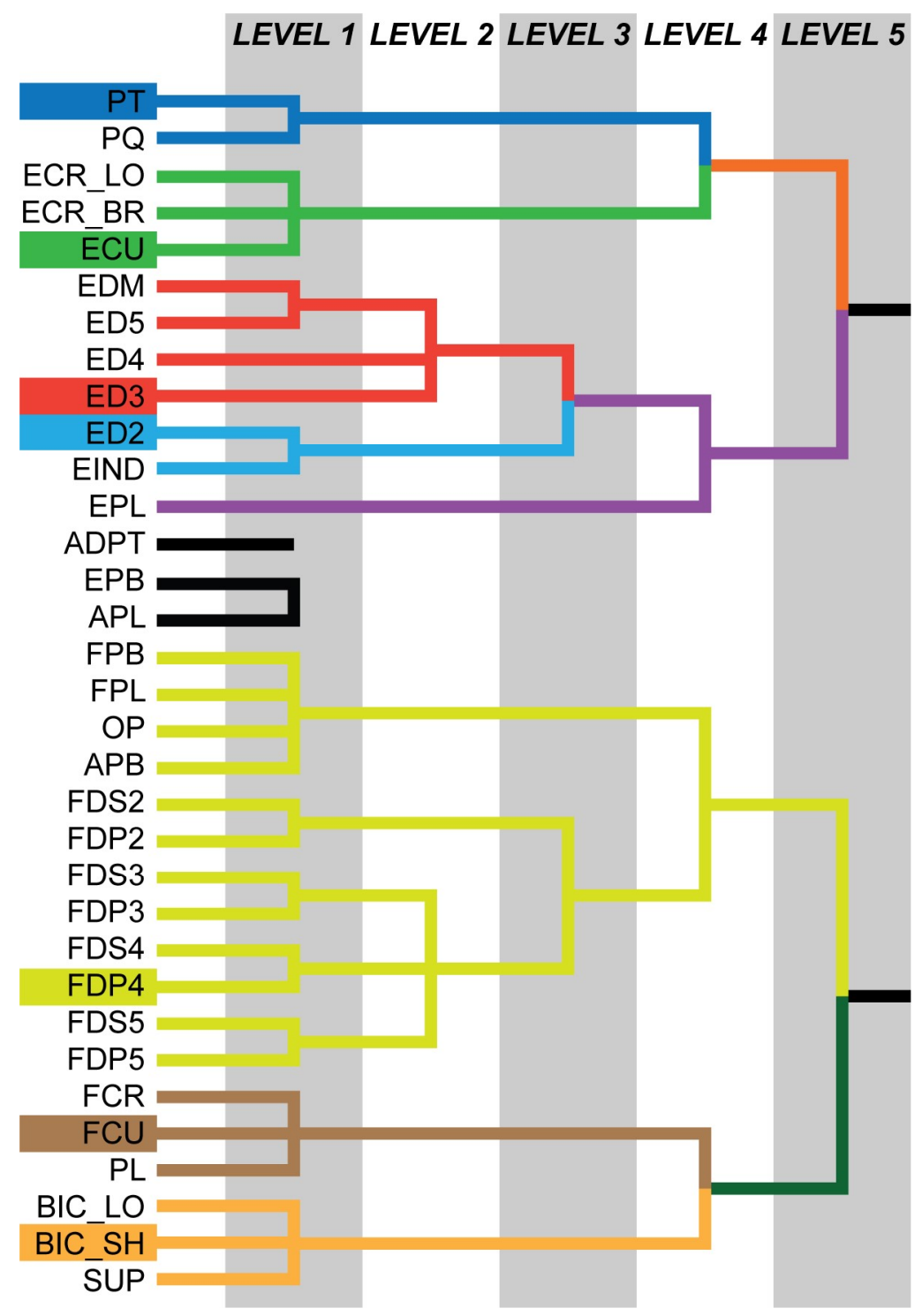

Fig. 3-3 Group Muscle Signal Processing Example Schematic. This diagram shows how the grouped controller provides signals to other muscles. The blocked colors on the left show signals that are recorded, and the lines show how that signal is provided to others that are not recorded. When two recorded signals connect, the signals are averaged to create a new signal and are sent to any muscles without a signal.

\subsubsection{Statistics}

We performed a hierarchical clustering analysis by first computing the Pearson's correlation coefficient between each combination of signal pairs and then calculating the heterogeneous 
variance explained (HVE). The HVE was computed as $1-R^{2}$ for $R<0$ and $1+R^{2}$ for $R \geq 0$. This metric identifies agonist muscle pairs by short HVE distances close to 0 and antagonist pairs close to 2. The average distance between all pairs of HVEs in any two clusters was used to create the hierarchical cluster trees shown in the results. We compared the structural similarity of the two hierarchical clusters, Gaussians and processed EMG, with the Fowlkes-Mallow similarity index (Fowlkes and Mallows, 1983). Since the clusters must have the same signals (i.e., same muscles), some of the Gaussian signals were not used in the comparison, as seen in Fig. 3-5. All statistical tests used an $\alpha$ value of less than 0.05 for statistical significance.

\subsection{Results}

In this study, we were able to calculate a participant's intended movement using recorded EMG and a musculoskeletal model and use it to move a real or virtual prosthesis. Processed EMG signals appear to look like noisy Gaussians. So we investigated whether we could use Gaussians as simplified control signals to replicate movement produced by EMG and see if there is any relationship between the Gaussians and EMG. During the Gaussian optimizations, we noticed that muscles tended to burst based on function. We used this idea to develop a method to supply control signals to muscles that are not recorded and tested the potential control online and offline. Videos of the movements are in the supplementary materials.

\subsubsection{Single Gaussian Optimizations}

We calculated single Gaussians for each muscle during different hand movements, which are described in Supplementary Table 3-3. We were able to find Gaussian signals that could produce a kinematic RMSE of less than 0.4 rad per DOF for 5 of the 10 movements we attempted. The 5 movements that did not resolve are listed in Supplementary Table 3-3, and the main cause was due to a single Gaussian not being able to create muscle co-contraction at the beginning and end of the movement to maintain a posture. Fig. 3-4 shows the joint angle results 
for the movement of flexing and extending the wrist and fingers while maintaining a neutral wrist pronation supination. Although the overall movement was performed, maintaining a constant DOF posture was problematic for the Gaussian signals, as can be seen in Fig. 3-4 for the cmc1 abd/add DOF. The results from the remaining 9 movements are shown in the supplemental material along with videos that demonstrate the desired movement against the simulated.

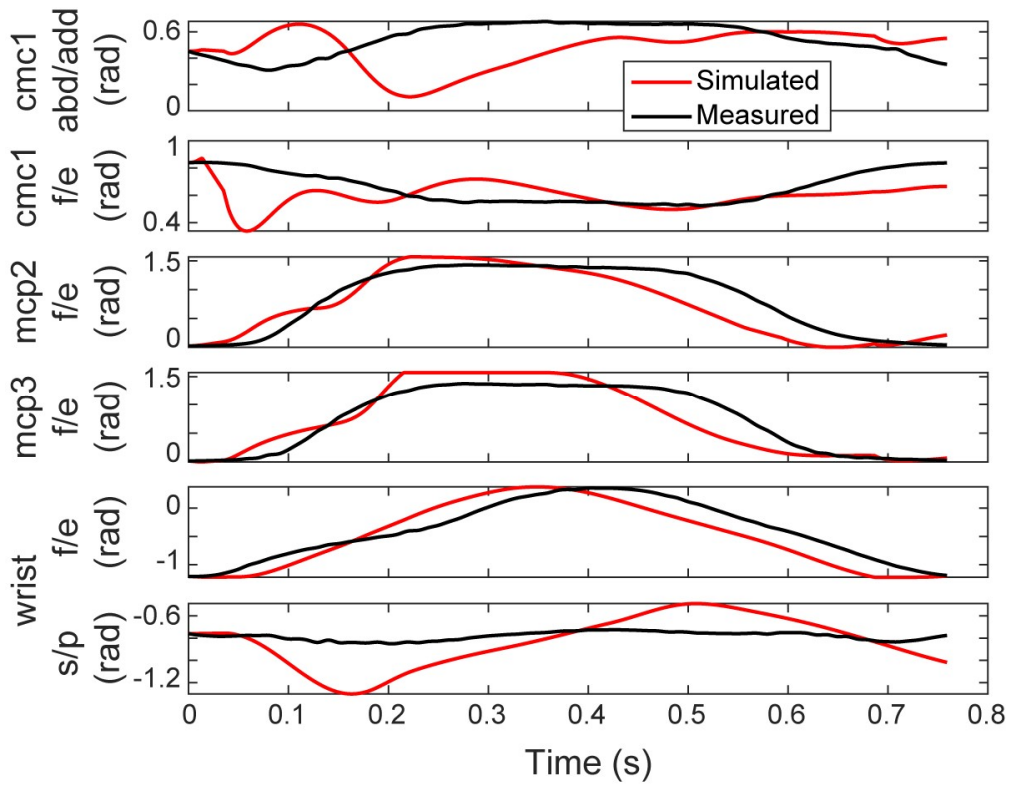

Fig. 3-4 Measured and Gaussian Simulated Joint Angles. The simulated (red) and recorded (black) kinematics were compared for a healthy participant. In this movement, the wrist and fingers were flexed and extended together, while the wrist pronation-supination and the thumb cmc1 joint maintained static posture.

We created hierarchical clusters of the processed EMG and Gaussians to see the relationships between muscles during the movement shown in Fig. 3-4. Supplementary material Table 3-2 provides additional information about the muscles and their abbreviations used in Fig. 3-5.

Qualitatively, there appears to be some logical structure, i.e., muscles with the same function activating together, that can be seen in Fig. 3-5 A and B for the recorded EMG. For example, BIC_LO and BIC_SH, as well as FDP2 and FDP3, burst together. Fig. 3-5 C and D contain the Gaussian signals for only the muscles that were recorded with EMG. A similar structure can be seen here, specifically referring to how the middle and index FDP compartments burst together.

Fig. 3-5 E and F shows the hierarchical cluster that is created with all the Gaussians signals. 
Here as well, there are muscles with the same function bursting together, i.e., FDS2 and FDS3, ECR_BR and ECU, PT and PQ, FPD3 and FDP2, etc. This process is expected if the

Gaussians signals can capture some of the structure that is embedded in the EMG.

A.

HVE

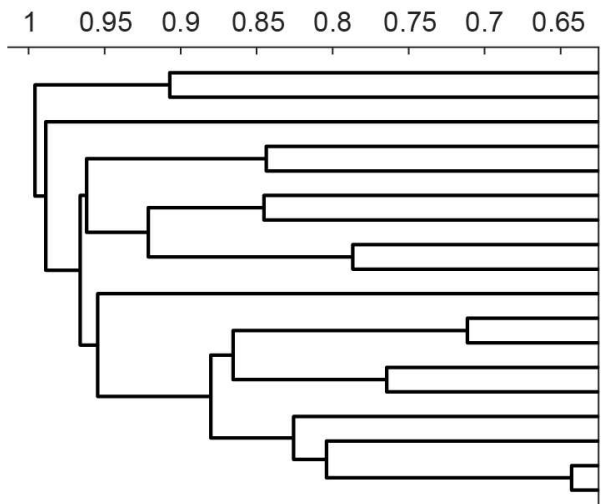

C.

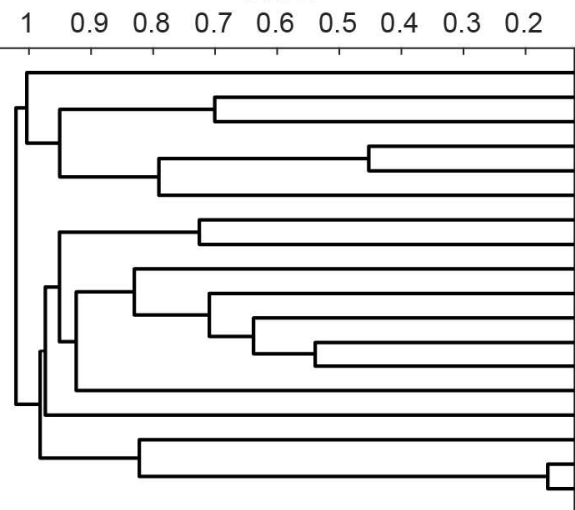

E.

HVE

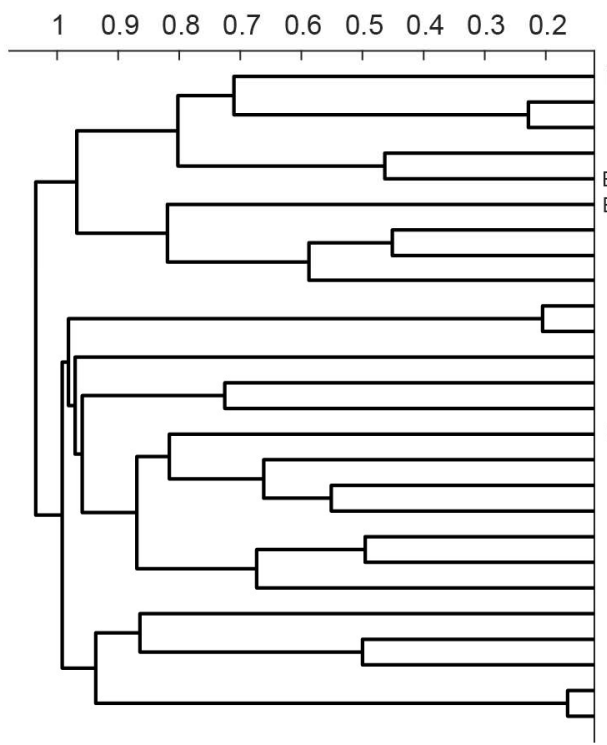

B.

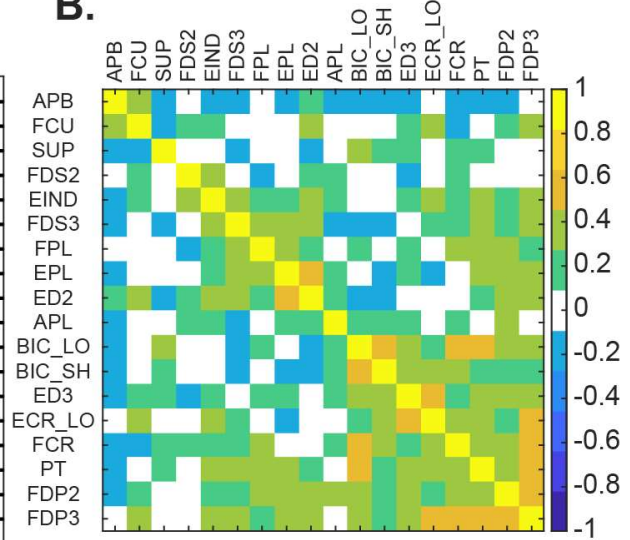

D.
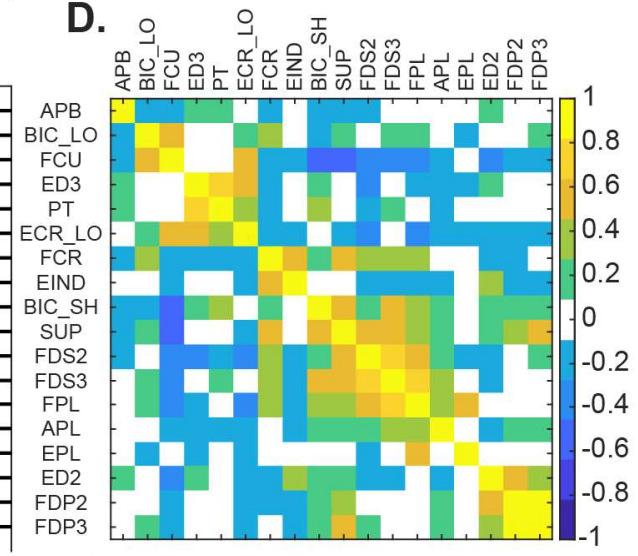

F.

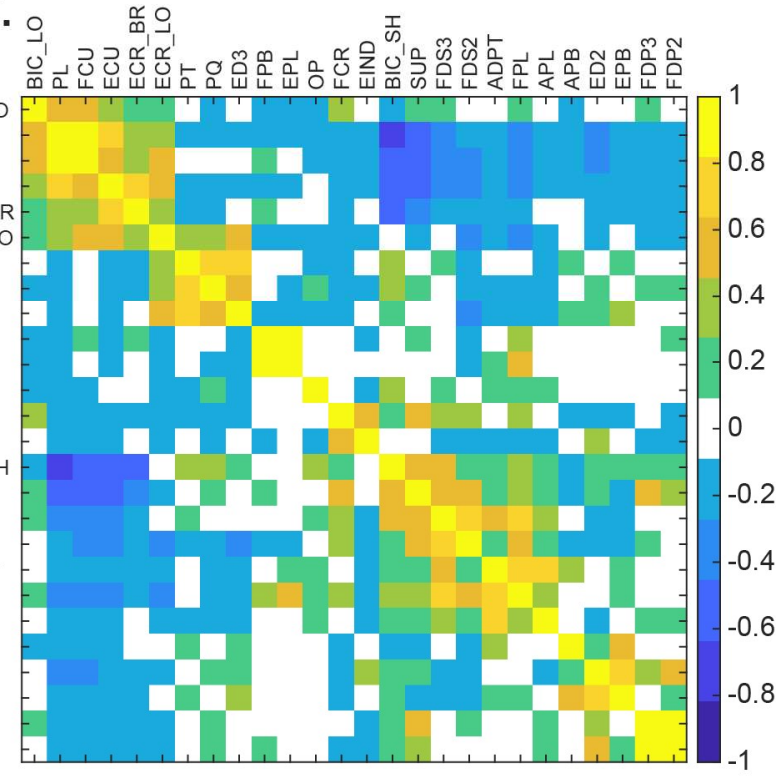


Fig. 3-5 HVE Clustering of Gaussian Signals and Recorded EMG. A, C, and E are cluster analyzes of HVE metrics of muscle excitation profile similarities and $\mathbf{B}, \mathbf{D}$, and $\mathbf{F}$ are the correlation matrixes of $r$-values between the muscle excitation profiles. The data in $\mathbf{A}, \mathbf{B}$ are recorded EMG, C, D are the Gaussian signals for hand muscles only, and $\mathbf{E}, \mathbf{F}$ are the Gaussian signals for all muscles in the model. Muscle labels and descriptions are in Table 3-2. The shown analysis is for the movement described in Fig. 3-4.

We wanted to see a quantitatively see if the Gaussian signals we computed contained any structural similarities to the recorded EMG. We did this by comparing the hierarchical clusters shown in Fig. 3-5 A and C using the Fowlkes-Mallow index. (Fowlkes and Mallows, 1983). Fig. 3-6 shows the Fowlkes-Mallow similarity index between the Gaussian and EMG hierarchical clusters at different maximum cluster groups. The similarity index is above average noise for each cluster, with cluster groups 2, 3, 4, 8, and 9 appearing above the interquartile range. The noise was calculated by permuting the EMG and Gaussian hierarchical clusters 1000 different times and calculating the Fowlkes-Mallow index each time. We empirically calculated a p-value between the EMG and Gaussian similarity index and the noise for each cluster number and used Fisher's method (Fisher, 1925) to evaluate the distribution of p-values. We found that cluster similarity was greater than random chance, which means that the Gaussians signals contained some amount of the structure embedded in the EMG. It shows that the optimizations worked to create signals that produce patterns like EMG. The issue is that it did not work for all 10 movements. Performing a similar analysis with a combination of 2 Gaussians per muscle could allow the signals to be complex enough to generate all the movement. 


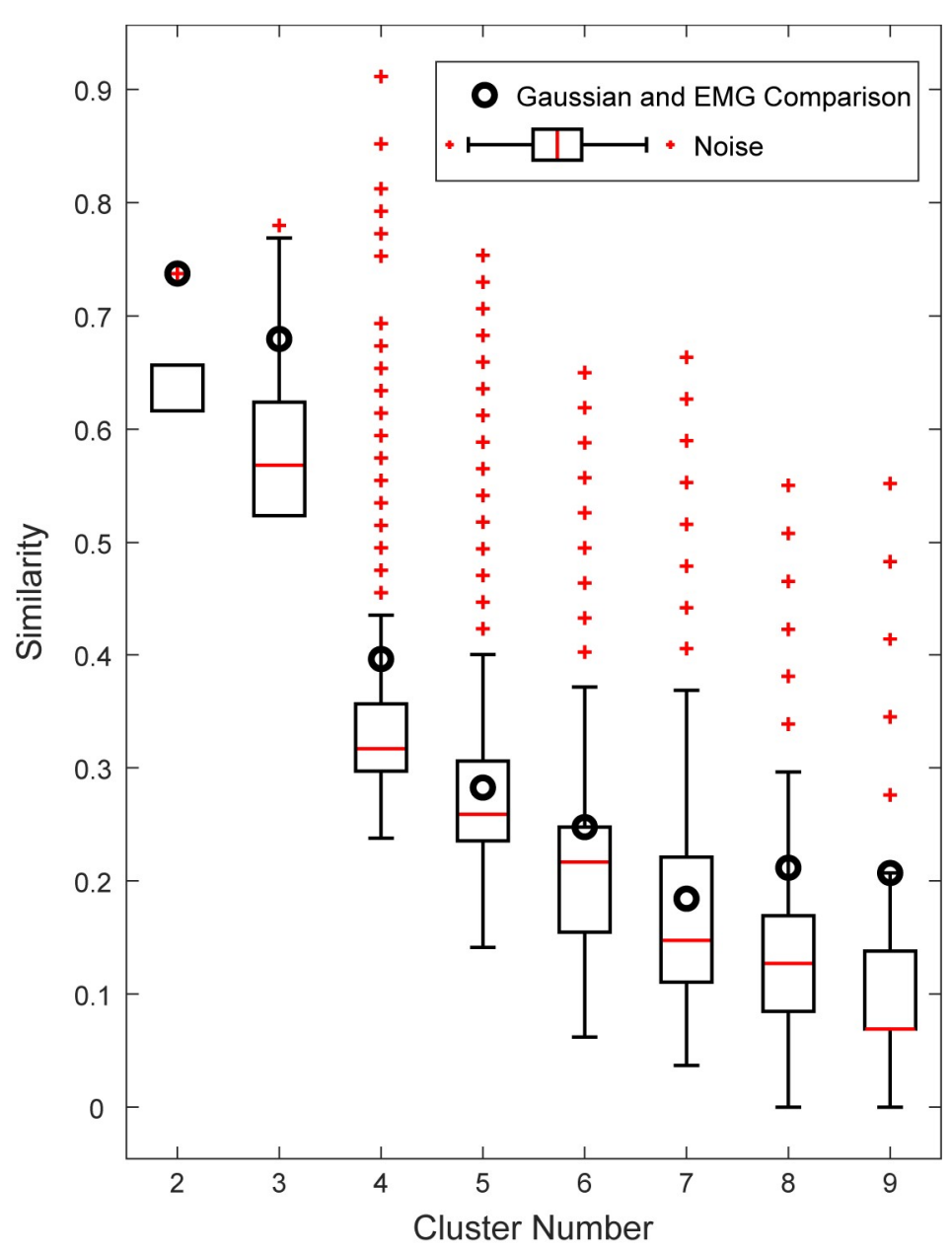

Fig. 3-6 Comparison of EMG and Gaussian Hierarchical Clusters. The x-axis shows the number of clusters used to create the hierarchical clustering. The y-axis is the Fowlkes-Mallow similarity index for the cluster comparisons, where 0 is completely different, and 1 is the same. The black circle is the similarity index between the Gaussians and processed EMG. The box plots show the level of spurious similarity (noise), which was created by randomly bootstrapping and comparing the relationships between the EMG and Gaussian hierarchical clusters 1000 different times.

\subsubsection{Grouped Muscle Signal Processing}

In the supplementary materials, there are videos showing the grouped signal processing running online matching movements of a healthy participant. There are 3 videos that help to understand how the processing works to add dexterity based on the number of muscles being recorded. When only two muscles are recorded, one flexor and one extensor, the control of the model is limited to hand open or closed. When there are four muscles being recorded, it opens up another DOF for independent control. The four types of muscles being recorded in the video 
are a wrist flexor, a wrist extensor, an index flexor, and an index extensor. The control achieved through these muscles is wrist flexion and extension separated from the hand opening and closing. There is also a video with 8 muscles being recorded, which are a wrist flexor, a wrist extensor, a wrist pronator, a wrist supinator, an index flexor, an index extensor, a thumb extensor, and a thumb flexor. This allows 4 different DOFs to be controlled simultaneously from recording data from 8 muscles. There is an additional video that has the model with the grouped signal processing controlling a single DOF (hand open is closed) 3D printed prosthesis.

Fig. 3-7 shows the kinematic results for two DOFs in hand, supplying data offline through the controller. This figure was created by taking the EMG data for the healthy individual and the amputee and running it through the controller offline with the grouped muscle signal processing to supply data to muscles not recorded. The movement was the wrist and finger flexionextension shown in Fig. 3-4. In Fig. 3-7, we chose to show only the wrist flexion-extension and index metacarpal flexion-extension because they represent the movement. For both the amputee and the healthy individual, the movement can be seen happening, where there is flexion and then extension. There is a video of both movements being simulated offline in the supplementary material. The offline kinematics do not appear promising; however, the videos of live control show a different story. There is potential that the offline data has some muscles mislabeled, which would deteriorate the result since it would supply incorrect data to other muscles. 

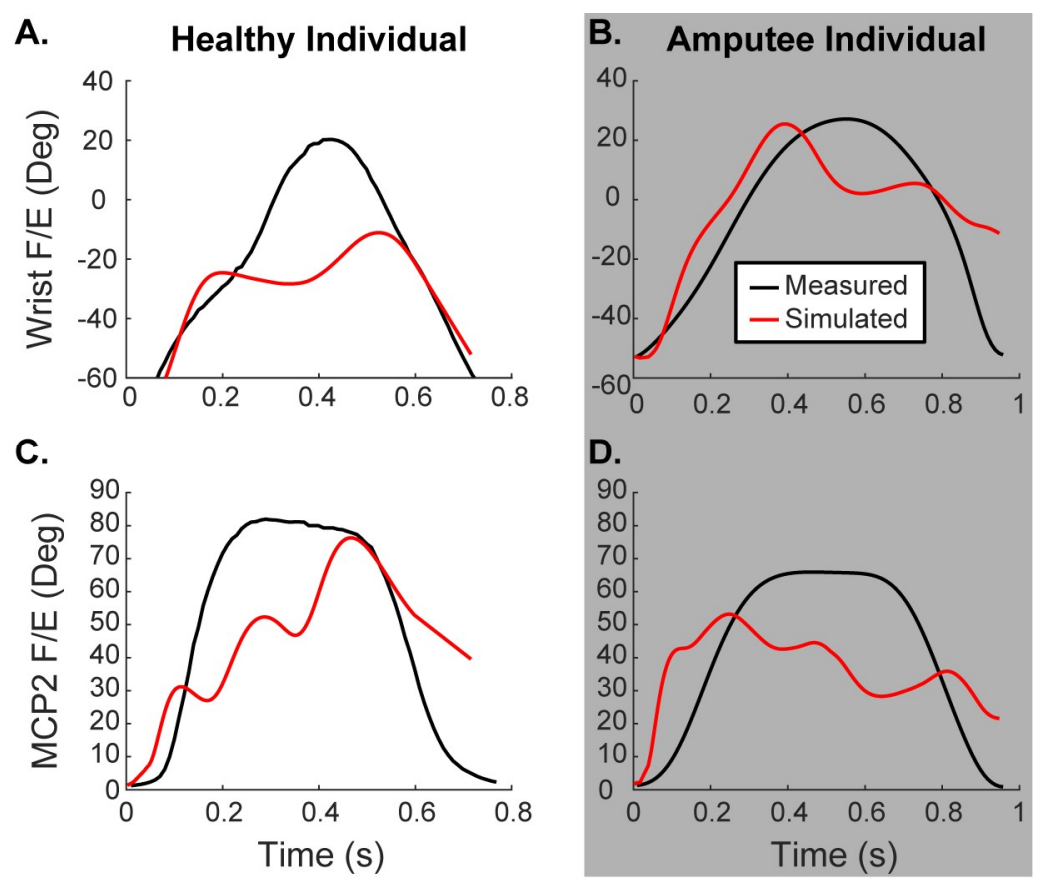

Fig. 3-7 EMG Driven Control with Muscle Group Processing. The black lines are measured kinematics for the healthy individual $(\mathbf{A}$ and $\mathbf{C}$ ) and instructional kinematics for the amputee (B and $\mathbf{D})$. The red line is the intended movement calculated offline, sending EMG through the biomimetic controller using the group signal processing. The grey box highlights the amputee results.

\subsection{Discussion}

In this study, we used a musculoskeletal model to estimate movement intent and control an upper limp prosthetic hand in real-time. In offline simulations, we were able to generate Gaussian signals that captured some of the embedded structures observed in muscle activity between muscles and used them as inputs to control simulated hand movements. In both online and offline simulations, we used the grouped signal processing to supply non-recorded muscles with control signals. This approach succeeds in supplying muscles with data; however, if any muscles are mislabeled, then it could supply the wrong information to multiple muscles causing deterioration of the overall control. The supplementary material videos show the successful deciphering of movement intent from healthy participants with minimal control signals.

Although previous work has shown controlling a prosthetic hand with a musculoskeletal model (Pan et al., 2018b), our work differs from what has been previously done. They only had 2 
movements in their model, which were wrist flexion/extension and finger MCP flexion/extension, while we showed control of an 18 DOF hand (See Supplementary Table 3-1 for simulated DOFs) that has natural physiological couplings to make 8 independent movements. The 8 movements were as follows: index through pinky finger flexion/extension, thumb flexion/extension, thumb abduction/adduction, wrist flexion/extension, and wrist pronation/supination. Pan et al. also simplified the musculoskeletal model by assuming a constant moment arm for each muscle-DOF relationship, and we chose to keep the posture dependent moment arms to keep the model accurate to human physiology. We used dynamic moments arms so that the muscle force and exertion required to move the prosthesis would be natural because hand posture is a large component involved in the amount of torque that a muscle can produce (Gonzalez et al., 1997). The dependence on posture can make a large difference in muscle force required to move the hand at the extremes of the ROM. The overall goal is to make the prosthesis as intuitive for the user as possible.

Another approach to making an intuitive prosthetic controller is machine learning. The standard machine learning approach for controlling a myoelectric prosthesis is to use an EMG classifier. This process requires recording training data of EMG and associated movements (e.g., hand open, hand close, wrist supination, wrist pronation, thumb extension) along with the need to recalibrate the system (Birdwell et al., 2015; Smith et al., 2014). The main issue with training datasets is ensuring that there is enough data to calculate the correct movement intent. The general problem stems from the fact that machine learning classifiers perform well for the posture that the training data was recorded in, but it degrades in other postures. This means that if the training data was recorded with the hand in a horizontal position, then the classifier could have issues if the arm is vertical. The musculoskeletal model approach does not have this issue because it is modeling the posture of the hand and applies muscle forces about that 
posture. It is a benefit of the musculoskeletal model approach not to have the system degrade in postures that have not been recorded.

Different prostheses require a different number of DOFs to be moved, and they can range from 1 DOF gripper (i.e., open/close) to a 6 DOF hand (e.g., luke hand (Resnik et al., 2018a)) and in the future potentially even more DOFs. It is essential that the prosthetic control approaches can be applied to any of these prosthetic hands. Our approach meets this criterion because it deciphers movement intent and then allows that movement to be mapped to any prosthesis. To control a high DOF prosthesis effectively requires the control of multiple DOFs independently. The number of independent movements our model can make depends on the muscles that can be recorded. At most, our method can achieve 8 independent movements: index through pinky finger flexion/extension, thumb flexion/extension, thumb abduction/adduction, wrist flexion/extension, and wrist pronation/supination. With the grouped signal processing, to have these movements be independent, it requires at least 1 agonist-antagonist muscle pair per movement for a total of 16 muscles. For example, to control wrist supination/pronation, it would require a wrist supination muscle and a wrist pronation muscle. The issue for the grouped signal processing involves the placement of the EMG sensors and ensuring that the correct muscles are recorded. For surface EMG sensors, there is also the issue of cross-talk where one sensor could record data from multiple muscles at once. The problem of cross talk can be bypassed with the use of intramuscular EMGs, where the sensor is inserted into the desired muscle.

Future work will investigate the potential to create a transformation between processed EMG and simplified control signals. The goal is to remove some of the inherent noise found in EMG by going from processed EMG to computed muscle activations. We started the first step in this process by created optimized Gaussian signals that could generate simulated hand movements. While these signals could simulate simple actions, it was not possible to perform movements that need co-contraction, which is required to stiffen a joint. For example, to keep the wrist in the 
same posture during finger movements, the wrist agonist-antagonist muscles both activate at the same time, causing co-contraction, which stiffens the wrist joint. Nonetheless, we generated control patterns composed of single Gaussians to represent the coordinated activation patterns for all simulated muscles. The spatial and temporal characteristics of each Gaussian were optimized to improve movement performance. To our surprise, the artificial patterns were correlated with experimental EMG, as indicated by Fig. 3-6. This was encouraging because a simple transformation could be possible once co-contraction can be achieved by simplified control signals. One method to simulate co-contraction would be to include a second Gaussian in the simplified signal to mirror the bursting pattern seen in some muscles at the beginning and end of movements. The inclusion of two Gaussians for each signal would ideally address the issue of co-contraction and, more importantly, resolve more complex burst patterns that would allow us to create simple transformations for EMG. The process of performing the optimizations again for 2 Gaussians was left to future work.

The main limitation of this study was ensuring that the correct muscle was being recorded. When using surface or inner-muscular EMG, it is difficult to guarantee that you are recording from the desired muscle in a healthy person, let alone an amputee who has altered or missing nerves and/or muscles. We combated this issue by having the participant perform movements and empirically deciphered the muscles that we were recording from based on the sensor placement and data. EMG cross-talk and the co-contraction of antagonistic muscle groups result in the correlated activity of muscles, which impede the identification. Future work would involve creating a system capable of deciphering the muscles being recorded.

In conclusion, we were able to create a prosthetic control system using the full description of a hand musculoskeletal model. We showed that simplified signals could be used to produce realistic movements. Our grouped signal processing was able to provide signals to muscles that were not recorded, which allowed us to decipher movement intent. Lastly, we have 
demonstrated that a fully described musculoskeletal model should be incorporated into any prosthetic controller, and the next step is to combine it with machine learning to achieve better control than either can alone.

\subsection{Supplementary Material}

The supplementary materials were submitted with the dissertation. It includes additional plots and videos for the chapter. There are videos of a healthy participant using the musculoskeletal model to control a 3D-printed hand. 
Table 3-1. The list of simulated DOFs. The id is a unique identifier for each DOF in the table, and it is used in Table 3-2 to show the DOFs each muscle spans. The name for each DOF is broken up into 4 parts: <Limb $>_{-}<$Joint $>_{-}<$Min $\rangle_{-}<$Max $>$. The first part is Limb, which is the placeholder for the limb where the joint is located each joint in this table is on the right arm (ra). The second is the short joint name, i.e., wrist (wr), carpometacarpal (cmc), metacarpophalangeal (mcp), interphalangeal (ip), proximal interphalangeal (pip), and distal interphalangeal (dip). Each finger joint has a number at the end of the joint name to indicate its corresponding finger, i.e. thumb (1), index (2), middle (3), ring (4), and pinky (5). The last two parts Min and Max correspond to the name of the rotation and the position of the joint at the minimum and maximum of the range, i.e. if the joint is wrist flexion/extension and extension was negative then the Min would be e, and the Max would be $f$. The abbreviations for the rotation name are supination (s), pronation ( $p$ ), flexion ( $f$ ), extension (e), adduction (add), and abduction (abd). The range is in radians and lists the minimum value and the maximum value for the joint. The last column describes the joints function.

\begin{tabular}{l|l|ll|l} 
id & Name & Range, rad & Description \\
\hline 1 & ra_wr_s_p & -1.5708 & 1.5708 & right arm wrist pronation/supination \\
2 & ra_wr_e_f & -1.2217 & 1.2217 & right arm wrist flexion/extension \\
3 & ra_cmc1_f_e & 0 & 0.8727 & right arm thumb proximal flexion/extension \\
4 & ra_cmc1_ad_ab & 0 & 0.8727 & right arm thumb proximal abduction/adduction \\
5 & ra_mcp1_f_e & -0.7854 & 0 & right arm thumb central flexion/extension \\
6 & ra_ip1_f_e & -1.5708 & 0 & right arm thumb distal flexion/extension \\
7 & ra_mcp2_e_f & 0 & 1.5708 & right arm index proximal flexion/extension \\
8 & ra_pip2_e_f & 0 & 2.0944 & right arm index central flexion/extension \\
9 & ra_dip2_e_f & 0 & 1.5708 & right arm index distal flexion/extension \\
10 & ra_mcp3_e_f & 0 & 1.5708 & right arm middle proximal flexion/extension \\
11 & ra_pip3_e_f & 0 & 2.0944 & right arm middle central flexion/extension \\
12 & ra_dip3_e_f & 0 & 1.5708 & right arm middle distal flexion/extension \\
13 & ra_mcp4_e_f & 0 & 1.5708 & right arm ring proximal flexion/extension \\
14 & ra_pip4_e_f & 0 & 2.0944 & right arm ring central flexion/extension \\
15 & ra_dip4_e_f & 0 & 1.5708 & right arm ring distal flexion/extension \\
16 & ra_mcp5_e_f & 0 & 1.5708 & right arm pinky proximal flexion/extension \\
17 & ra_pip5_e_f & 0 & 2.0944 & right arm pinky central flexion/extension \\
18 & ra_dip5_e_f & 0 & 1.5708 & right arm pinky distal flexion/extension
\end{tabular}


Table 3-2. The list of simulated musculotendon actuators. The id is a unique identifier for each muscle. The name is the abbreviated name for the muscle, with the next column being the full muscle names. The DOF column lists the id's for the DOFs the muscle spans, see Table 3-1 for DOF names. The next column lists the 3 muscle length muscle parameters in meters: minimum muscle length $\left(L_{\min }\right)$, muscle length threshold for passive forces to kick in $\left(L_{\text {pass }}\right)$, and the maximum muscle length $\left(L_{\max }\right)$. Minimum and Maximum lengths were found from the raw data. The last column lists the maximum active muscle force $\left(F_{\max }\right)$ and the maximum passive muscle force $\left(F_{\text {pass }}\right)$. The maximum muscle force was found through the process described in Chapter 2.

\begin{tabular}{|c|c|c|c|c|c|c|}
\hline id & Name & Full name & DOFs & $L_{\min } L_{\text {pass }} L_{\max },(m)$ & \multicolumn{2}{|c|}{$F_{\text {pass }} F_{\max },(N)$} \\
\hline 1 & BIC_LO & $\begin{array}{l}\text { Biceps brachii long } \\
\text { head }\end{array}$ & 1 & 0.3280 .4330 .445 & 10.92 & 154.3 \\
\hline 2 & BIC_SH & $\begin{array}{l}\text { Biceps brachii short } \\
\text { head }\end{array}$ & 1 & 0.2480 .3540 .366 & 10.92 & 154.3 \\
\hline 3 & SUP & Supinator & 1 & 0.0680 .0860 .088 & 25.55 & 361.0 \\
\hline 4 & $\mathrm{PT}$ & Pronator teres & 1 & 0.2170 .2350 .237 & 23.1 & 317.2 \\
\hline 5 & $P Q$ & Pronator quadratus & 1 & 0.0220 .0330 .041 & 12.25 & 168.2 \\
\hline 6 & ECR_LO & $\begin{array}{l}\text { Extensor carpi radialis } \\
\text { longus }\end{array}$ & 12 & 0.3160 .3460 .349 & 14 & 136.4 \\
\hline 7 & ECR_BR & $\begin{array}{l}\text { Extensor carpi radialis } \\
\text { brevis }\end{array}$ & 12 & 0.3150 .3480 .352 & 17.15 & 167.1 \\
\hline 8 & ECU & Extensor carpi ulnaris & 12 & 0.3180 .3340 .336 & 12.25 & 119.3 \\
\hline 9 & FCR & Flexor carpi radialis & 12 & 0.2870 .3320 .337 & 18.2 & 54.2 \\
\hline 10 & $\mathrm{FCU}$ & Flexor carpi ulnaris & 12 & 0.2940 .3270 .331 & 35 & 104.2 \\
\hline 11 & PL & & & & & \\
\hline 12 & FDS5 & $\begin{array}{l}\text { Flexor digitorum } \\
\text { superficialis (pinky } \\
\text { finger) }\end{array}$ & 21617 & 0.3120 .3650 .371 & 7.35 & 58.4 \\
\hline 13 & FDS4 & $\begin{array}{l}\text { Flexor digitorum } \\
\text { superficialis (ring } \\
\text { finger) }\end{array}$ & 21314 & 0.3260 .3810 .387 & 8.4 & 57.7 \\
\hline 14 & FDS3 & $\begin{array}{l}\text { Flexor digitorum } \\
\text { superficialis (middle } \\
\text { finger) }\end{array}$ & 21011 & 0.3350 .3930 .399 & 14.7 & 109.1 \\
\hline 15 & FDS2 & $\begin{array}{l}\text { Flexor digitorum } \\
\text { superficialis (index } \\
\text { finger) }\end{array}$ & 278 & 0.3340 .3860 .391 & 12.6 & 117.8 \\
\hline 16 & FDP5 & $\begin{array}{l}\text { Flexor digitorum } \\
\text { profundus (pinky finger) }\end{array}$ & 2161718 & 0.3290 .3860 .392 & 8.75 & 69.6 \\
\hline 17 & FDP4 & $\begin{array}{l}\text { Flexor digitorum } \\
\text { profundus (ring finger) }\end{array}$ & 2131415 & 0.3440 .4020 .409 & 12.95 & 89.1 \\
\hline 18 & FDP3 & $\begin{array}{l}\text { Flexor digitorum } \\
\text { profundus (middle } \\
\text { finger) }\end{array}$ & 2101112 & 0.3560 .4160 .422 & 14.35 & 106.5 \\
\hline 19 & FDP2 & $\begin{array}{l}\text { Flexor digitorum } \\
\text { profundus (index } \\
\text { finger) }\end{array}$ & 2789 & 0.3470 .4090 .415 & 14.35 & 134.1 \\
\hline 20 & EDM & Extensor digiti minimi & 2161718 & 0.3730 .3950 .423 & 5.25 & 78.7 \\
\hline 21 & ED5 & $\begin{array}{l}\text { Extensor digitorum } \\
\text { (pinky finger) }\end{array}$ & 2161718 & 0.3720 .3980 .424 & 1.75 & 26.2 \\
\hline
\end{tabular}




\begin{tabular}{|c|c|c|c|c|c|c|}
\hline 22 & ED4 & $\begin{array}{l}\text { Extensor digitorum } \\
\text { (ring finger) }\end{array}$ & 2131415 & 0.3860 .4140 .441 & 4.2 & 122.1 \\
\hline 3 & ED3 & $\begin{array}{l}\text { Extensor digitorum } \\
\text { (middle finger) }\end{array}$ & 2101112 & 0.3960 .4290 .458 & 5.95 & 109.0 \\
\hline & ED2 & $\begin{array}{l}\text { Extensor digitorum } \\
\text { (index finger) }\end{array}$ & 2789 & 0.3970 .4310 .458 & 3.85 & 52.9 \\
\hline & EIND & Extensor indicis & 2789 & $\begin{array}{lll}0.231 & 0.261 & 0.292\end{array}$ & 4.55 & 62.6 \\
\hline & EPL & $\begin{array}{l}\text { Extensor pollicis } \\
\text { longus }\end{array}$ & 124356 & 0.2430 .2740 .294 & 6.65 & 402.7 \\
\hline & EPB & Extensor pollicis brevis & 2435 & 0.1680 .1790 .199 & 4.55 & 42.9 \\
\hline & FPB & Flexor pollicis brevis & 435 & 0.0790 .1010 .104 & 4.55 & 34.9 \\
\hline$y$ & FPL & Flexor pollicis longus & 24356 & 0.2300 .2640 .289 & 17.85 & 136.9 \\
\hline & APL & $\begin{array}{l}\text { Abductor pollicis } \\
\text { longus }\end{array}$ & 1243 & 0.1660 .1810 .198 & 13.65 & 128.9 \\
\hline & OP & Opponens pollicis & 43 & 0.0580 .0640 .064 & 10.15 & 77.8 \\
\hline & APB & Abductor pollicis brevis & 435 & 0.0590 .0740 .076 & 5.25 & 40.2 \\
\hline & ADPT & $\begin{array}{l}\text { Adductor pollicis } \\
\text { transversus }\end{array}$ & 435 & 0.0320 .0610 .064 & 3.15 & 141.4 \\
\hline
\end{tabular}

Table 3-3. Movements. The id column is a unique identifier for each movement. The next column describes the movement and what the DOFs were meant to do. The last column gives a yes or no response to if the single Gaussian's could resolve the movement to a kinematic RMSE of less than 0.4 rads per DOF.

\begin{tabular}{|c|c|c|}
\hline id & Movement Description & $\begin{array}{c}\text { Gaussian } \\
\text { Performed } \\
\text { Movement }\end{array}$ \\
\hline 1 & thumb flex/ext, fingers neutral, wrist ext, wrist rotation neutral & No \\
\hline 2 & thumb abd/add, fingers neutral, wrist ext, wrist rotation neutral & No \\
\hline 3 & thumb flex/ext, fingers neutral, wrist flex neutral; wrist rotation supinated & No \\
\hline 4 & thumb neutral, fingers neutral, wrist flex/ext, wrist rotation neutral & Yes \\
\hline 5 & thumb neutral, fingers neutral, wrist flex neutral, wrist rotation pro/sup fast & Yes \\
\hline 6 & thumb neutral, fingers and wrist flex/ext fast, wrist rotation neutral & Yes \\
\hline 7 & thumb neutral, fingers flex/ext slow, wrist flex neutral, wrist rotation neutral & No \\
\hline 8 & thumb neutral, fingers neutral, wrist flex/ext slow, wrist rotation neutral & Yes \\
\hline 9 & thumb neutral, fingers neutral, wrist flex neutral, wrist rotation pro/sup slow & No \\
\hline 10 & thumb neutral, fingers and wrist flex/ext slow, wrist rotation neutral & Yes \\
\hline
\end{tabular}




\section{Chapter 4 Self-paced Omnidirectional Locomotion in Virtual Reality Using a Human-in-the-Loop Robotic System}

(this chapter is in preparation to be submitted to Science Robotics as "Boots M, Yakovenko S (2019) Self-paced Omnidirectional Locomotion in Virtual Reality Using a Human-in-the-Loop Robotic System.")

\subsection{Abstract}

Our bodies are complex machines fine-tuned for moving through often unpredictable environments. The biological control pathways spanning cortical and spinal networks compute the visuomotor transformation from visual inputs to motor outputs. The inverse transformation from output to input could be embedded into the robotic devices to enable intuitive interfaces in human-in-the-loop (HIL) active robotic systems. For omnidirectional navigation, this biomimetic HIL implementation requires the computation of heading direction from the observed motor output generated by the spinal patterning circuits. The inverse model of these circuits implicates limb speeds as the control variables; thus, connecting them as inputs to the independent belts of a treadmill should enable self-paced locomotion. The heading direction can then be coded into virtual reality as the turning function of limb speeds closing the loop between a human and a machine. The validity of computations was tested in naïve subjects asked to reduce interlimb step symmetry or match heading directions during walking. Our system allowed robust omnidirectional control and improved step symmetry when desired. This result supported the general validity of a theoretical visuomotor inverse. This biomimetic HIL system is a holistic tool for the functional exploration of human locomotor control principles. 


\subsection{Introduction}

From an engineering perspective, humans are high dimensional complex machines that have the dexterity that has yet to be matched by current robotics. However, when a person is injured and loses functionality, we turn to robotics to provide that functionality back. This solution has led to the creation of highly sophisticated prostheses and other restorative devices. An example of a sophisticated device is a spinal cord stimulator that provides a person that could not walk after a spinal cord injury the ability to walk again (Carhart et al., 2004; Wagner et al., 2018). The walking is not completely natural but still provides back the basic desired functionality. There have been better solutions with below-knee prostheses that are powered and can help provide back a natural gait (Au et al., 2008, 2007; Grimmer et al., 2016); however, when the prosthesis gets more complex, such as a hand, the desired natural control is harder to achieve (Cordella et al., 2016). This problem has led to solutions that lack graceful movement execution, but still, are very promising because they provide back the basic needed functionality.

Human locomotion, although a seemingly simple task that is intuitive for us to do, is an overwhelmingly sophisticated control problem when we think about Bernstein's degree of freedom problem (Bernstein, 1967; Domkin et al., 2002). It is an issue of motor redundancy and how humans or animals can perform the same movement in a variety of ways to obtain the same goal. This fact is a challenge for machine learning when finding a solution that can work for multiple people that might do things differently. We believe that machine interfaces can be improved by adding physiological processes into the control structure defined as the biomimetic approach. The goal is to model the appropriate physiological process that will help the machine interface be more intuitive for a human. The first step to applying a biomimetic approach to locomotion is understanding the physiological processes that are involved. The person decides on a direction to walk, deemed heading direction, and this sends signals down that person's supraspinal pathways to the spinal locomotor central pattern generator (CPG), which are 
responsible for spinal rhythmogenesis (Brown, 1911; Grillner, 1985; Rybak et al., 2015).

Moreover, this neural structure is a target of both feedforward and feedback control pathways involved in the initiation and regulation of gait. It has never been shown what the signals that enter the spinal CPG encode, but it has been shown that limb velocity could be involved (Yakovenko et al., 2018). We used this theory and incorporated it into a self-paced treadmill design to look at human locomotion.

In this study, we developed a self-paced treadmill robot that works with a human-in-the-loop to allow people to walk in a virtual environment. The common approach to a self-paced treadmill is to create a feedback system that keeps the user in the middle of the treadmill based on their position (Jonghyun Kim et al., 2013; Lichtenstein et al., 2007; Minetti et al., 2003; Sloot et al., 2014b; Souman et al., 2011; Stavar et al., 2011). These systems do not allow inter-limb speed differences seen in natural walking due to the belts maintaining the same speed. Our approach is to use the output of the spinal CPG walking stance and swing periods to make a split-belt treadmill self-paced with independent limb speed control. Fig. 4-1 shows a basic diagram of how the system works with a human. The user decides on a direction to walk in the virtual environment, and the system attempts to simulate the desired direction and speed. The system was inspired by the locomotor CPG and the self-paced treadmill works like an inverse CPG to calculate the person's leg speeds. The error of the system is the difference between $\gamma$ and $\gamma^{*}$ in Fig. 4-1, and by decreasing this error, the walking will feel more realistic. To support this idea, we tested the user's control of the system with three locomotor tasks that involved the user's treadmill speed control and their individual leg speed control. If the participants can show control of the system without ever previously working with it, then this supports the idea that humans control their limbs with a velocity related signal. This device can be used as a tool to study the different aspects of human locomotion. 


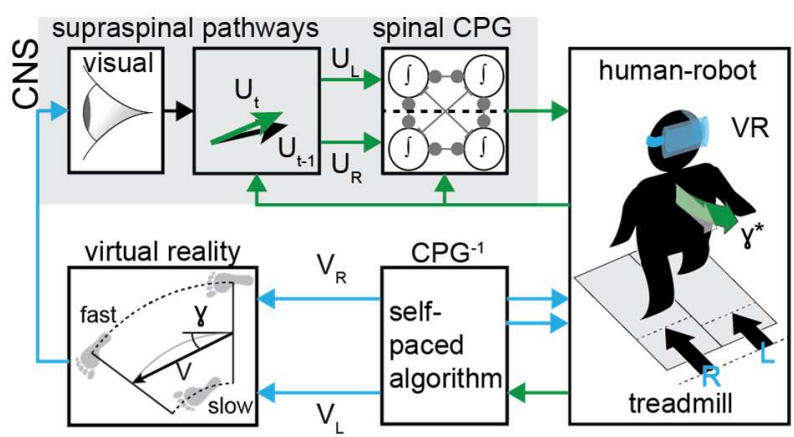

Fig. 4-1 Self-paced Treadmill Concept Diagram. This diagram shows the flow of information and how it interacts with the biological system of a human. By matching the velocity signals out of the self-paced algorithm with the signals out of the supraspinal system makes the system feel natural for the user.

\subsection{Materials and Methods}

\subsubsection{Locomotor Tasks}

We tested the participant's ability to walk on the self-paced treadmill through 3 locomotor tasks. The first tested the participant's ability to match desired speeds and accelerations on the selfpaced treadmill. The second asked the participants to walk symmetrically from an asymmetric condition with and without visual flow. It tested if the visual feedback was appropriate and helped them to be more symmetrical. The last task tested their ability to control their individual leg speeds to perform active turning tasks in a virtual environment. The participants completed these tasks without any prior experience with the system. The specific tasks are described in more detail below.

The first task tested their ability to match desired speeds and accelerations. Fig. 4-4A shows the visual the participants were provided on a screen in front of the treadmill, which provided them a visual of their current speed (black line) and the desired speed (black circles). The red dot was provided in the middle to help keep the participant focused looking directly in front of them. The profile shown in Fig. 4-4B displays the user's ability to match the five steady speeds $(0.5,0.75$, $1.0,1.25$, and $1.5 \mathrm{~m} / \mathrm{s}$ ). At least twenty steps per subject were collected at each steady-state 
speed. We collected data of three different accelerations $\left(0.01,0.02\right.$, and $\left.0.05 \mathrm{~m} / \mathrm{s}^{2}\right)$ shown in Fig. 4-4C-E. Each acceleration profile had a different number of ramps to collect at least twenty steps accelerating and decelerating. The goal was to have people be able to match their speed to the desired speed to within 10 percent of the desired.

The second task asked participants to walk symmetrically after starting from a fixed asymmetric gait. There were three randomized trials performed for each participant, where the only difference between the trials was the starting asymmetric belt speeds. The starting belt speeds held the left belt at $1 \mathrm{~m} / \mathrm{s}$ for each trial with the right belt being either $1.1,1.2$, or $1.3 \mathrm{~m} / \mathrm{s}$. Fig. 4-5A shows the order of events that happened during each trial. The participant walked for 15 steps on each leg in a fixed asymmetric condition without any visual feedback. Then we made the treadmill self-paced and asked the participants to walk symmetrically and report when the belt speeds were symmetrical. The participants were asked to not look at the belts during the experiment. We visually monitored them and reminded them to not look at the belts but straight ahead anytime they looked down. Once the participant reported that the belts were symmetrical, we collected 20 more steps on each leg. After 40 steps (20 each leg) of "symmetrical" walking, we provided visual feedback about the user's gait on a screen mounted to the wall in front of them. The visual feedback was provided through a VR environment with a textured ground and arrows showing the participant's current heading direction, Fig. 4-6A without the triangle. The participants used this information to report again when they were walking symmetrically, and we proceeded to collect another 20 steps on each leg before concluding the trial.

The third locomotor task tested the participant's ability to control their leg speeds to match a heading direction in VR. The task asked the participants to match 8 different heading direction changes, $-80,-60,-40,-20,20,40,60$, and 80 degrees. These heading directions were repeated 7 times and provided to the participant in a random order, creating a total of 56 heading direction changes for each participant to complete. The heading changes were 
provided based on the participant's current heading direction when the trial started. This means at the beginning of the trial the desired heading direction was set by adding the participant's current heading direction and the specific heading change. Fig. 4-6A was shown to the participant to provide them feedback on their heading direction (blue arrows) and the desired heading direction (red triangle). The participant was asked to change their heading direction to match that of the desired heading direction shown in Fig. 4-6B. Once the participants heading direction matched the desired, shown when the blue arrows entered the triangle and turned the triangle green, the participant needed to maintain that heading direction for two seconds. Then the next heading direction was provided. The participant had 20 seconds to complete a trial before it was considered a failed attempt. This task was repeated twice, once with the visual feedback provided on screen in front of the participant and once with the participant wearing the VR headset.

\subsubsection{Protocol}

Volunteers ( $\mathrm{N}=15,8$ females, 7 males, $24.8 \pm 4$ years old) without a history of musculoskeletal trauma or neurological disorders participated in this study. All participants walked on a split-belt treadmill in a safety harness. Of the 15 volunteers, eight participated in locomotor task 1 (4 females, 4 males), nine participated in locomotor task 2 ( 4 females, 5 males), and five participated in locomotor task 3 (3 females, 2 males). All procedures used in the study were approved by the WVU IRB (protocol \# 1605135438).

\subsubsection{Hardware}

We developed a self-paced treadmill using a split-belt treadmill (Bertec) instrumented with 6dimensional force sensors under each belt. The stepping velocities of each leg were calculated using belt loading signals using a custom algorithm in MATLAB (release 2018b, MathWorks). The real-time communication linked limb speed calculations to the changes in the heading 
direction in virtual reality (VR) developed with a standard gaming engine (Unreal Engine 4) connected to either a VR headset (HTC Vive, v.1.0) or high-definition 60-inch display placed in front of the treadmill.

\subsubsection{Control System}

Each treadmill belt has its speed calculated independently of the other belt. Fig. 4-2 depicts the flow diagram for the control process. Ground reaction forces and the current treadmill speed are used in the algorithm to calculate the speed of each leg (U). Then, the user's leg speed was adjusted to provide an appropriate belt speed to maintain the user in the middle of the treadmill. The last step in the process implemented hazard rules to detect if the user fell and stop the treadmill if that happened.

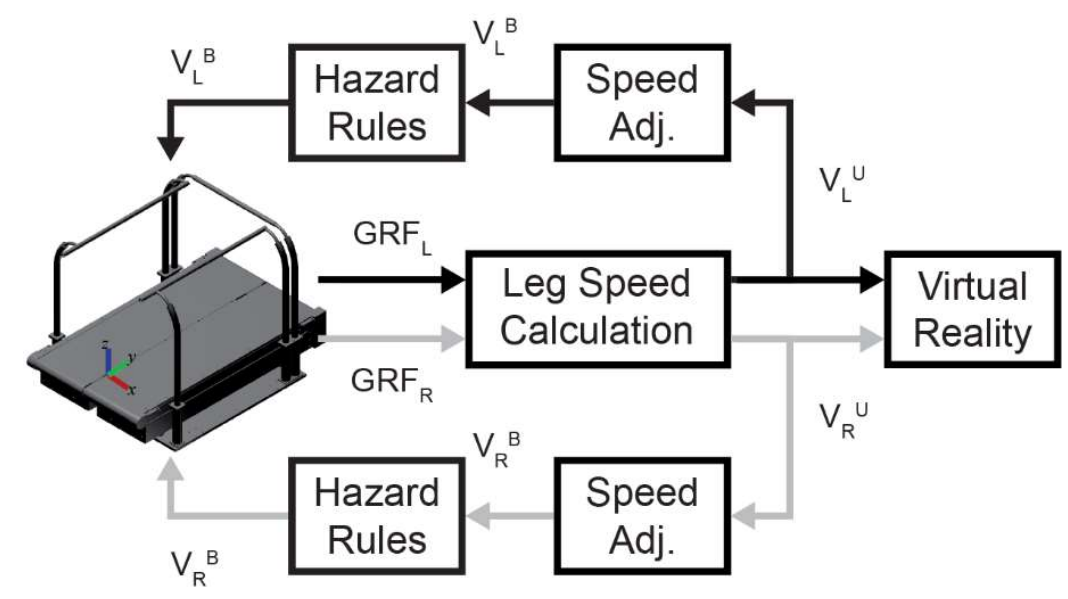

Fig. 4-2 Control flow diagram. This diagram shows the flow of data from sensors to belt speed calculations. The GRF stands for ground reaction forces, where each GRF sensor provides 6 signals (force $[N]$ about $x, y, z$, and moments $[N m]$ about $x, y, z$ ). The black arrows are the left belt, and the grey arrows are the right belt. Speed Adj. stands for speed adjustment, $\mathrm{V}^{\mathrm{U}}$ is the user's leg speed, and $\mathrm{V}^{\mathrm{B}}$ is the treadmill belt speed.

\subsubsection{Leg Speed Calculation}

The step-behind method calculated leg speed based on the ratio of the distance your foot traveled to the time of the step cycle. As the name of the method implies, the leg speed was updated after one full step cycle and caused a delay in calculating the new belt speed. This 
calculation was performed at the onset of the stance in the step cycle (Fig. 4-3A, $t^{O N}$ circles). The leg speed calculation was the distance traveled from onset to onset divided by the step cycle time (Equation 4-1). The distance was calculated as the integral of the treadmill belt speeds from onset to onset with an added distance term to account for the subject's forward/backward movement on the treadmill (Fig. 4-3B). The $\Delta y$ is described in Equation 4-2 as the difference between the current y onset location and the previous. The y-onset values, used in Equation 4-1 through 4-3, were found by using the maximum forward location of the foot on the treadmill during the stance phase of a step, shown in Fig. 4-3A. Similarly, the y-offset values were found at the minimum forward location on the treadmill. The stance phase of a step was determined from thresholding the vertical ground reaction force at $50 \mathrm{~N}$ to produce the $t^{O N}$ and $t^{O F F}$ values in Fig. 4-3A.

\section{Equation 4-1}

$$
V_{i}^{U}=\frac{\int_{t_{i-1}^{O N} V_{i}^{B} d t+\Delta y}^{t_{O N}}}{\mathrm{~T}},
$$

where $V^{B}$ is the velocity of the treadmill belt, $t^{O N}$ is the time of events marking step onsets, $\Delta y$ is the distance traveled forward on the treadmill, $\mathrm{T}$ is the step cycle time, $i$ is the step counter, and $V^{U}$ is the user's calculated leg speed.

\section{Equation 4-2}

$$
\Delta y=y_{i}^{O N}-y_{i-1}^{O N}
$$

where $y^{O N}$ is the foot location forward on the treadmill at step onset. All the variables in Equation 4-1 through 4-3 are labeled in Fig. 4-3 to show where they occur in the data. 


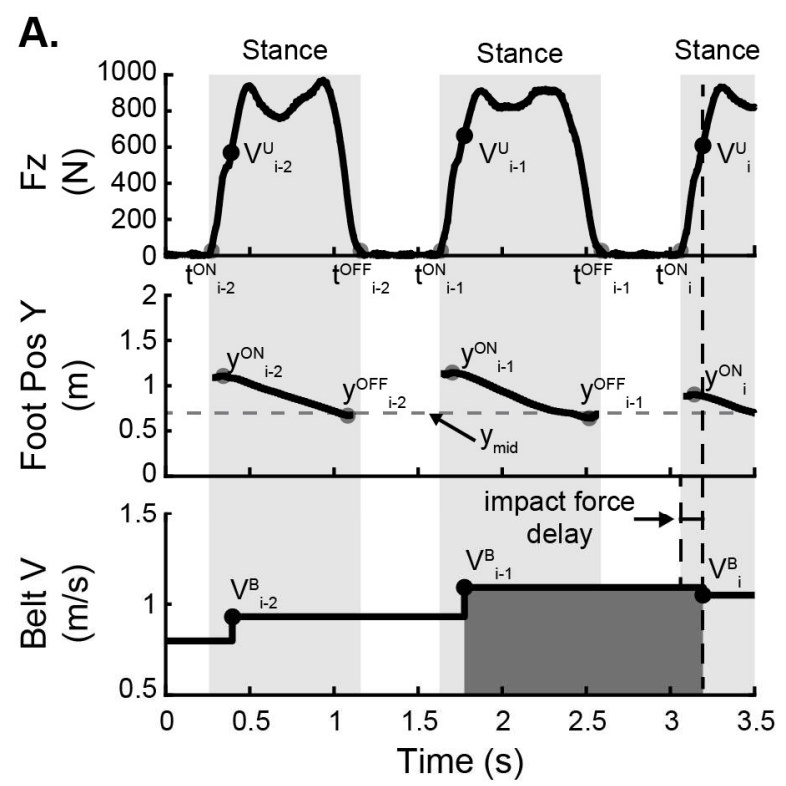

B.

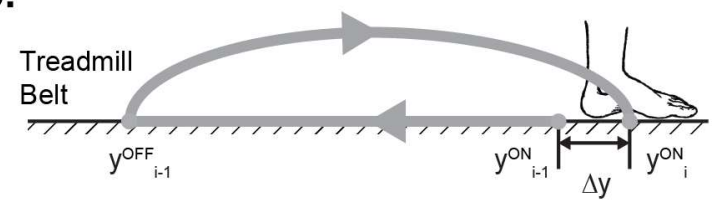

Fig. 4-3 Leg Speed Calculation. (A) A speed calculation example of how the leg speed was calculated from the ground reaction force data. It used the Fz force to find onsets and offsets in the data (top row). The black circle shows that there is a wait time after onset to have a negligible step impact so that onset to onset speed calculation can be performed. The second row shows the foot position with the y-onset value being the maximum value forward on the treadmill, with the y-offset value being the minimum. The bottom row is the belt velocity, which is different from the user's leg velocity. (B) This figure shows where the foot is located in space during the calculation. The $\mathrm{y}^{\mathrm{ON}}$ marks onset of stance, the $\mathrm{y}^{\text {OFF }}$ marks offset of stance, $\mathrm{i}$ is the step counter, and $y$ is the foot position forward on the treadmill. This figure is a visual of the movement forward or backward on the treadmill described in Equation 4-2.

\subsubsection{Speed Adjustment}

Fig. 4-3B shows that there is movement forward and backward on the treadmill during the leg speed calculation. This movement is due to the user changing their speed and the treadmill adjusting its speed at the next step onset. To move the user back to the middle of the treadmill, we made the belt speed be different from the user's speed. The difference is shown in Equation 4-3, where the belt speed is a combination of the user's current leg speed and the speed adjustment based on the user's average leg location during the last leg stance. 


\section{Equation 4-3}

$$
V_{i}^{B}=V_{i}^{U}+\frac{\frac{1}{2}\left(y_{i-1}^{O N}+y_{i-1}^{O F F}\right)-y_{m i d}}{T}
$$

where $V^{B}$ is the belt speed, $V^{U}$ is the user's leg speed, $y$ is the foot location forward on the treadmill, $O N$ is the onset of stance, $O F F$ is the offset of stance, $i$ is the step counter, $y_{\text {mid }}$ is the central location of the treadmill, and $\mathrm{T}$ is the step cycle time.

\subsubsection{Hazard Rules}

We developed event detection to keep the user safe on the treadmill. Hazard rules are added to the output of all estimates to overwrite the treadmill belt speeds when hazard states are detected. The hazard states we implemented were to stop the treadmill if something is going wrong. The main hazard rule is to stop the treadmill if the user moves within $15 \mathrm{~cm}$ from the back of the treadmill. The main reason a person would move this far back on the treadmill would be due to falling, and in this instance, the treadmill stops before the user falls off the treadmill.

\subsubsection{Stepping Between Belts}

The leg speed calculation requires ground reaction forces for each leg, and it can be disrupted by foot placement in-between the belts. There is a force sensor for each belt, and it records the ground reaction forces for the corresponding leg. When a person steps in-between the belts, the data for one leg is recorded by both sensors. This occurrence impairs our leg speed calculations. We tracked the subject's foot positions with the ground reaction forces, while they are walking on the treadmill. These locations allow us to monitor when one foot moves toward the break between the belts. Right before the foot crosses and touches the two belts at once, we assume its y location based on Equation 4-4. We continue this process until we detect that the foot in question goes into the swing phase. However, if the subject is completely walking on one belt, then we cannot calculate accurate leg speeds because we cannot detect foot stance 
and swing phases in the data. In this instance, we reset the treadmill data, inform the subject to walk on both belts, and maintain the current belt speeds until we detect data for each leg on separate belts.

\section{Equation 4-4}

$$
y_{j}=y_{j-1}-V_{j}^{B} \cdot\left(t_{j}-t_{j-1}\right)
$$

where $y$ is the foot location forward on the treadmill, $V^{B}$ is the belt speed, $t$ is the data timestamp, and $j$ is the current data sample.

\subsubsection{Virtual Reality}

The self-paced treadmill was linked to virtual reality with Equation 4-5 and 4-6 to provide visual flow accurate to the user's leg speeds during walking. The visual flow was created with Unreal Engine 4 and was created to provide objects to move past the participant, shown in Fig. 4-6A. The visual was of a desert with a patterned ground as well as cacti provided for visual flow. The environment was created to have everything be realistically sized so that the movement in the environment would be as realistic as possible. The participant moved through the environment at a speed calculated by Equation 4-5. The diagram in Fig. 4-2 shows that the user's leg speeds were sent to the VR and not the treadmill belt speeds. The heading direction of the avatar was calculated by Equation 4-6. This angle was provided to the blue arrows that followed in front of the participant in Fig. 4-6A. The first arrow was the current heading direction and the following arrows provided an estimate of the path that would be walked if the heading direction was continued. The red cone for task 3 was created to follow in front of the avatar and provide the desired heading direction for the participant to walk. The virtual environment could be supplied to a screen in front of the treadmill or to a virtual reality headset (HTC Vive).

\section{Equation 4-5}

$$
V^{A}=\frac{1}{2}\left(V_{R}^{U}+V_{L}^{U}\right)
$$


where $V^{A}$ is the average user leg speed, $V^{U}$ is the leg speed, $R$ is the right leg, and $L$ is the left leg.

\section{Equation 4-6}

$$
\gamma=\frac{\left(V_{R}^{U}-V_{L}^{U}\right) \cdot\left(T_{R}+T_{L}\right)}{2 W}
$$

where $T$ is the step cycle time, $W$ is the distance between the feet during the stance of each leg, and $\gamma$ is the angular rate (rad/s) that the avatar is rotated during the movement.

\subsection{Results}

In this study, we developed a self-paced, split-belt treadmill for studying locomotion and linked it to VR to simulate turning and provide visual feedback. Participants were asked to complete three locomotor tasks to assess their control of the self-paced treadmill. The first task showed that the user could match desired walking speeds and accelerations, the second showed that the VR provided appropriate visual feedback to enable users to walk more symmetrically, and the third showed the user's ability to control active turning in VR by matching desired heading directions. The participants' completion of these three tasks demonstrated their level of control achieved from people without prior experience of the system.

\subsubsection{Locomotor Task 1: Self-Paced Treadmill Speed Control}

The first task asked users to match desired walking speeds and accelerations by showing them Fig. 4-4A, which displayed their speed and the desired speed. Fig. 4-4B-E shows an example participant's ability to match the desired speeds and accelerations for four different speed profiles. The user's ability to match the five steady speeds are shown in Fig. 4-4B with the 3 accelerations being shown in Fig. 4-4C-E. Fig. 4-4F-G shows the average RMS of the difference between the user's speed and the desired speed for all participants. The average values from our example participant's data Fig. 4-4B-E are shown in red in Fig. 4-4F-I. Fig. 4-4H-I shows the 
values from Fig. 4-4F-G normalized to the desired speed. We anticipated the self-paced treadmill to be controllable within $10 \%$ of the desired speed, which was met on the average subject for 10 of the 11 profiles ( 5 steady-state speeds and 6 accelerations). The only profile that did not meet this criterion was the $0.05 \mathrm{~m} / \mathrm{s}^{2}$ deceleration. As some participants reported, this may be caused by their reluctance to move backward on the treadmill to slow down. 

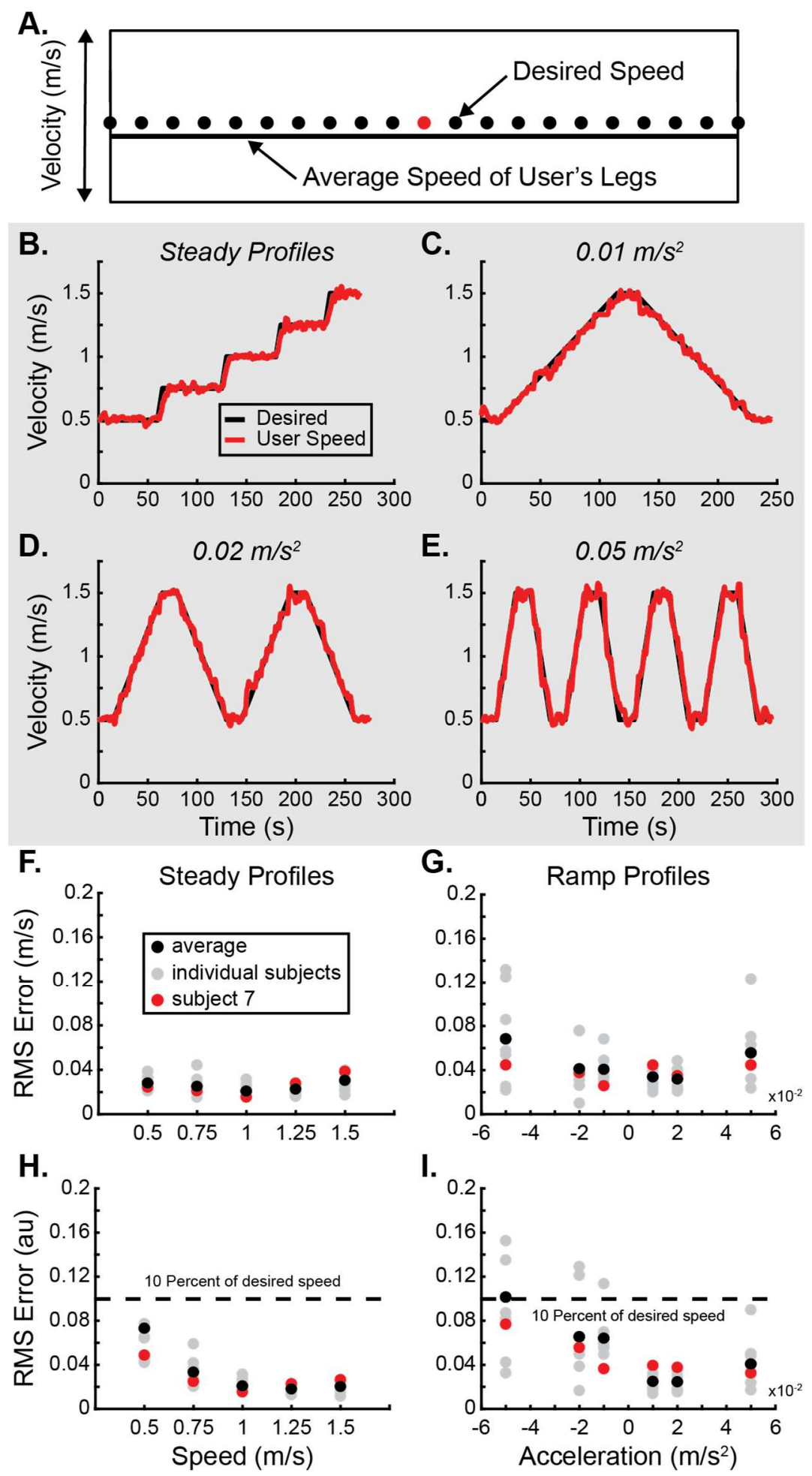

Fig. 4-4 Locomotor task 1 treadmill profile matching. (A) The participant was displayed this figure on a screen informing them of their speed and the desired speed. (B-E) Shows the walking profiles that the participants were asked to walk. The black line is the desired speed, and the red line is an example of user speed. (F-G) These plots show the RMS error between 
the desired speed and the participants. The red dots are from the example subject is shown in panels B-E. (H-I) These plots are F-G normalized to the desired speed, and they show that the average participant was able to control the treadmill speed to within 10 percent of the desired speed represented by the dashed line.

\subsubsection{Locomotor Task 2: Symmetrical Walking}

The second task asked participants to walk symmetrically after starting from one of three fixed asymmetric gaits. The data displayed in Fig. 4-5B are the 15 steps collected at the fixed speed, the 20 steps collected at the self-paced speed without VR, and the 20 steps collected at the self-paced speed with VR, each of these groups is shown in Fig. 4-5A. The grey dots in Fig. 4-5B are individual averages for each of the three trials. We ran a two-way repeated-measures ANOVA with 2 within-subject factors (start asymmetry and feedback conditions). The feedback conditions are fixed with no VR, self-paced with no VR, and self-paced with VR. We used a Tukey post hoc analysis to make the comparisons shown in Fig. 4-5B by the asterisks. It shows that the visual flow on average significantly helped the participants walk more symmetrically. The fixed speed condition has such a small speed difference distribution between the leg speeds due to the treadmill belts being fixed. 

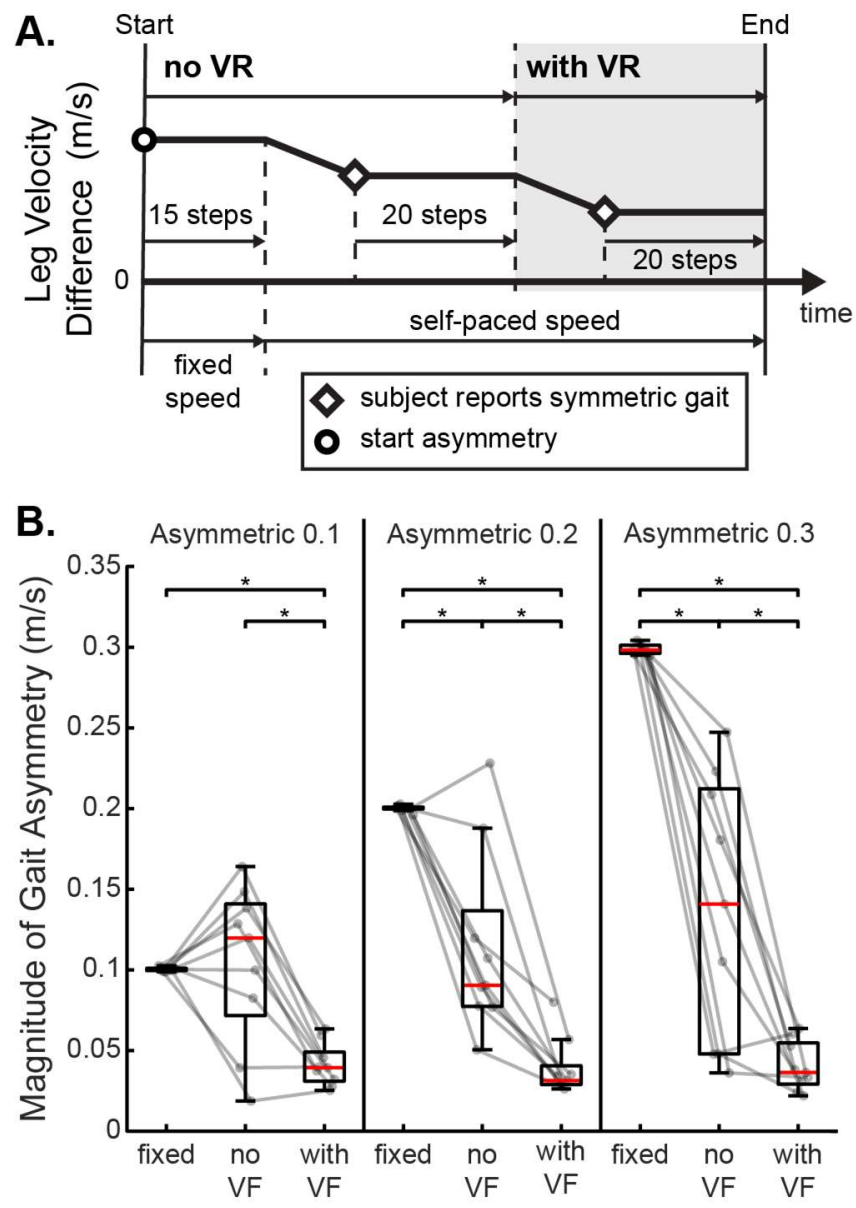

Fig. 4-5 Locomotor task 2 individual leg speed control. (A) Locomotor task 2 experimental design. The solid black line shows that the user has some natural asymmetric gait. The trial starts without VR and fixed belt speeds and ends with VR and self-paced belt speeds. The timing of these events are shown at the vertical lines. The beginning of the trail starts without VR and later has VR turned on to provide visual feedback about the user's leg speeds. (B) The three different trials are separated by the three columns. The differences are the start of asymmetric conditions. The grey dots and lines are individual subject averages. The box plot shows the overall performance of the subjects. The asterisks denote statistical significance $(\alpha<0.05)$.

\subsubsection{Locomotor Task 3: Active Turning}

The third locomotor task tested the participant's ability to control their leg speeds to match a heading direction in VR. Fig. 4-6A was shown to the participant to provide them feedback on their heading direction (blue arrows) and the desired heading direction (red triangle). Fig. 4-6C shows the individual and average participant success rates across heading directions.

Participants were able to successfully turn in both the on-screen and the VR headset tasks. For 
smaller angles (20 and $40 \mathrm{deg}$ ), the VR headset and on-screen tasks had similar completion rates (>90\%). However, for the larger angles (60 and $80 \mathrm{deg}$ ), we observed lower success rates in the VR headset tasks despite all participants indicating the VR headset felt more natural and easier to control in the follow-up questionnaire. The participants were asked to turn in the VR environment as fast as they could while maintaining control. Fig. 4-6D shows the average angular velocity for each subject fitted with a regression line to show the trend for on-screen and in the headset. This figure shows participants moved faster to reach larger heading directions, supporting what we would expect when asking someone to turn.

A.

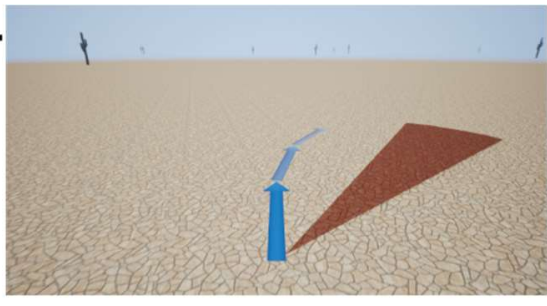

C.

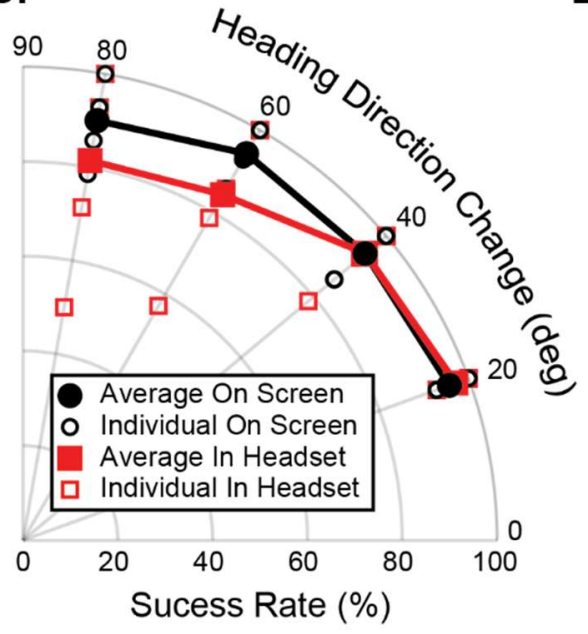

B.

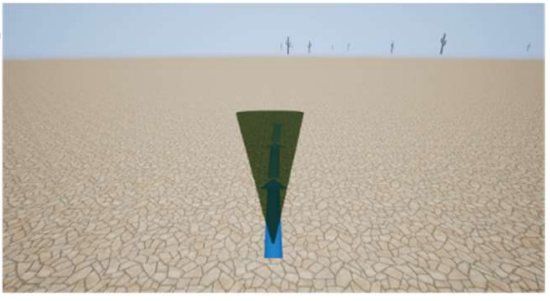

D.

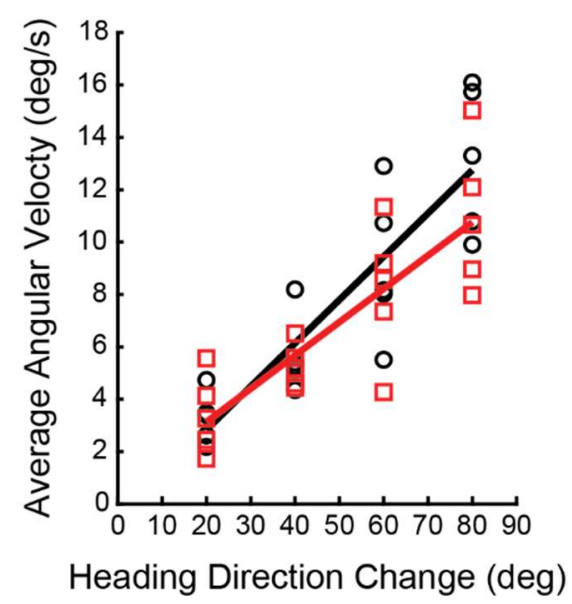

Fig. 4-6: Asymmetric locomotion in VR using a self-paced split-belt treadmill with a Headset or On-Screen presentation of heading direction. (A) Instruction to turn towards the red cone. (B) Successful completion of the trial (3 steps in the cone). (C,D) Performance averages per subject are shown. All subjects can control turning and scale the rate of turning with the required amplitude change. 


\subsection{Discussion}

The goal of this study was to create a congruent visuomotor experience on a self-paced treadmill. The system was able to allow participants to complete normal locomotor tasks intuitively, such as turning, because of the independent control of belt speeds. Additionally, participants were given a survey after the walking tasks that asked them to report on how natural the walking felt for each task. They reported that the tasks with the visual flow in the VR headset felt more natural than on the screen, supporting the notion that we provided appropriate visual feedback. The ability for each subject to control the treadmill easily and quickly without training could indicate that the self-paced treadmill works in a way that is intuitive for the operator.

The common approach to a self-paced treadmill is based on adjusting the treadmill speed to keep the person walking in the middle of the treadmill with a depth sensor (Jonghyun Kim et al., 2013; Minetti et al., 2003) or motion capture (Sloot et al., 2014b; Souman et al., 2011; Stavar et al., 2011). To the best of our knowledge, very few published self-paced treadmills reported a speed matching test, but one that did had similar results to ours in task 1 (Yoon et al., 2012). Like the other self-paced treadmills, ours involves keeping the person walking in the middle of the treadmill (see 4.4.5 Speed Adjustment), but there are two differences from the other systems involving how we calculate the leg speeds.

The first difference is that we calculate each limb speed independently, while the other systems only calculate a whole body speed. Keeping the limb speeds independent helps make the system more like natural over-ground walking, which allows for gait asymmetry, particularly during turning. There are two strategies used for turning: a step strategy, which our self-paced treadmill can simulate (see Fig. 4-6), and a spin or pivot strategy (Akram et al., 2010; Hase and Stein, 1999; Taylor et al., 2006). In terms of rehabilitation, it is important to have the capability to 
control the interlimb speed and simulate turning. The aim of rehabilitation is to improve motor function through the mechanisms of neuroplasticity. It has been shown that walking straight forward does not involve the motor cortex as much as more challenging tasks like stepping over obstacles (Drew et al., 2008) or turning. Therefore, rehabilitative tasks which are designed to necessitate the involvement of the motor cortex may facilitate motor recovery.

The second difference between our system and others is that our system works based on calculating leg stance and swing times from ground reaction forces. Motion capture data could also be used to calculate step phase times, but without motion capture, the user can walk on the treadmill without any additional instrumentation on them. Our quick, minimal setup, therefore, makes our system more viable for clinical adoption. Our system also provides visual feedback to the person walking, which can be used by clinicians to give feedback and tasks to the user.

The goal of a self-paced treadmill is to allow the user to change their speed and minimize the number of unnecessary accelerations and decelerations, so the walking feels natural. We approached the development of a self-paced treadmill keeping in mind how the biological system allows us to walk. The central nervous system contains spinal neural mechanisms called central pattern generators (CPGs) that are responsible for spinal rhythmogenesis (Grillner, 1985). Moreover, this neural structure is a target of both feedforward and feedback control pathways involved in the initiation and regulation of gait. In our laboratory, we have developed data-driven CPG models (Yakovenko et al., 2018) that predict the nature of at least some of the main inputs, which happens to be limb speed, to the spinal locomotor CPG. This inspired us to create a self-paced treadmill that worked by using leg stance and swing times along with current speed to calculate limb speed. We calculated the leg speed and adjusted the treadmill speed once a step. This limits the amount of treadmill speed changes, but the drawback is that the person walking moves slightly on the treadmill when they change their speed before the treadmill can adjust. 
Walking on a fixed speed treadmill differs from walking over-ground because it does not allow the participant to adjust their speed naturally, which can be corrected when using a self-paced treadmill (Plotnik et al., 2015; Sloot et al., 2014b). Moreover, adding visual feedback to treadmill walking has been shown to improve gait parameters to more closely match over-ground walking (Sloot et al., 2014a). It has even been shown that turning strategies can be seen in people walking on a treadmill if their visual flow is of turning (Oh et al., 2018). Because our system allows independent control of belt speeds, differences in limb speeds can be interpreted into visual flow heading direction. Our system attempts to replicate over-ground walking on a treadmill and provide a better platform for studying gait rehabilitation.

Matching the visual flow to the user's actions closes the loop on the robotic system, which places the human in the control loop with the machine. Future work will use this system to attempt to tease out the internal mechanisms used for locomotion. Current studies have shown that by performing treadmill gait adaptations while blindfolded helped reinforce the adaptations carry over to natural over-ground walking (Torres-Oviedo et al., 2011; Torres-Oviedo and Bastian, 2010). It would be interesting to use our self-paced treadmill system to test if gait adaptation retention improves when the participant has visual flow that matches the walking adaptation. Another interesting study would be to see if the self-paced treadmill can be used to allow people with an asymmetric gait to train to walk more symmetrically. Then see if that learned behavior can carry over to over-ground walking. Future work will show if this system can be used in gait adaptation rehabilitation.

The limitation of the biomimetic approach is that it can be hard to model the biological processes involved; however, if they are incorporated properly, the controller can become more intuitive. The main limitation of the self-paced treadmill is that it needs one full step to occur before it can calculate a leg speed. This process led to some movement forward and backward on the treadmill. Because there is movement forward and backward on the treadmill very fast 
accelerations or decelerations, such as going from slow walking to immediately sprinting, can cause the treadmill speed adjustment to feel unnatural. This is due to having great movement forward or backward on the treadmill. To mitigate this issue, future work will incorporate a feedforward estimate of the speed based on ground reaction forces to get an estimate of the current steps speed during push-off.

In this study, we showed that a biomimetic approach could be applied to create a self-paced treadmill with independent limb speed control. We were able to show how well participants have control over the treadmill belt speeds. Our system allowed for more realistic control that could match overground walking due to the ability to have different limb speeds, which allows for step strategy turning, and its ability to provide appropriate visual feedback. This system can be used as a tool to investigate human locomotion and improve gait rehabilitation.

\subsection{Supplementary Materials}

The supplementary materials were submitted with the dissertation. It includes videos of two participants performing locomotor tasks. It also includes additional videos in virtual environments. 


\section{Chapter 5 Discussion and Future Directions}

My dissertation explores the process of controlling restorative robotics by embedding physiological processes into the control approach to improve human-machine interfaces. Specifically, my research focused on improving prosthetic hand controllers and split-belt treadmills for gait rehabilitation. I chose these two different systems to show that a biomimetic approach can be applied to a variety of restorative robotics that involve different biological rehabilitation. The second chapter goes through the development and validation of a musculoskeletal model of the forearm and hand that can be used for real-time prosthetic control. The third chapter used the developed musculoskeletal model to simulate movement intent and control a physical or virtual prosthetic hand. In the fourth chapter, we switched our focus to the control of a split-belt treadmill, where the controller is inspired by the human spinal central pattern generator used for walking. Participants were able to control individual belt speeds of the self-paced, split-belt, treadmill and performed various walking tasks with minimal training. To summarize, using a biomimetic approach can be advantageous for the control system in restorative robotics.

\subsection{Musculoskeletal Models}

In Chapter 2, we developed a method to validate a musculoskeletal model and ensure it is as physiologically accurate as possible. Our process was a direct validation approach that involved the iterative comparison of the muscle paths to previously published data. The more common approach is to validate the model for a specific task indirectly. An example of an indirect validation would be the comparison of simulated and recorded muscle activity patterns that produce the same movement (de Zee et al., 2007). The indirect approach is more commonly used because it is challenging and often costly to acquire the data for a direct comparison (Lund 
et al., 2012). We chose the direct validation approach because it allowed us to validate the structure, i.e., muscle path, of each muscle individually.

We had to use multiple published datasets to create our dataset for direct validation. Often in the published datasets, moment arms were measured from people of different sizes and genders, leading to inherited variability within individual and complete datasets. Moreover, the moment arms available in literature only contained data for a subset of possible postures. To address the dataset sparseness, we developed evaluation tools to identify common model failures, e.g., moment arm zero-crossing due to muscle's wrapper geometry failures in extreme positions. The example in Fig. 2-4 depicts an error in the ECU muscle during extension, where the moment arm sign to flipped from extension to flexion, producing an inconsistency. Fig. 2-7 shows 31 zero crossings before the validation was reduced to 10 expected zero crossings. The evaluation tools allowed us to maintain accurate muscle-DOF relationships throughout the full ROM. Fig. 2-6 shows our qualitative metric indicating the model's improvement. Although some moment arm errors about the thumb were successfully identified, they were not corrected due to the complex interactions about the CMC joint.

Using either a direct or indirect validation process still requires additional validations that examine overall function. The reason is that even if the structure is validated, the forces being produced are not necessarily physiologically accurate, i.e., the simulation produced the appropriate movement, but the muscle forces generated are not necessarily physiologically possible. We accomplish functional validation by scaling the Fmax muscle parameter (see Equation 3-1) until simulated and experimental joint torques are in agreement (see torque dataset). The scaling of muscle force addressed possible inconsistencies in the measured moment arm dataset and the muscle model simplification of a lumped force parameter. Furthermore, the estimated maximum forces are estimated by multiplying specific tension and PCSA. The issue with this estimate is that there are many different published values for both 
PCSA and specific tension. This problem requires the scaling of the Fmax parameter to ensure the muscle is producing physiological muscle forces.

After we completed the functional validation, we wanted to verify that our new lumped maximum force parameters were reasonable. We verified our values by calculating muscle specific tension using published PCSA values (Chao et al., 1989; Happee and Van der Helm, 1995) and found that the specific tension was not significantly different from published experimental values (see Fig. 2-8B). These results further support the validity of our simulated muscle force generation. The specific tension values calculated from 4 other musculoskeletal models (Buchanan, 1995) were significantly higher than experimentally published values (see Fig. 2-8 for model references). The most likely reason for the discrepancy between our model's specific tension and the other models is due to our model having a functional validation. The goal of the two-step validation process was to create a generic hand model designed to represent an average healthy adult by having its torque output and muscle paths match with published average data.

We showed the importance of an accurate musculoskeletal model by answering the question, "What do we lose when we assume constant moment arms instead of their true dynamic nature for the wrist flexion/extension DOF?" The constant moment arm assumption is accurate in the middle of the ROM, but it becomes inaccurate towards the extremes (Fig. 2-9A grey shaded regions), on average, approximately $17 \pm 14 \%$ from the constant moment arm values. We asked the question because although musculoskeletal models have been shown to work for prosthetic control (D. Crouch and Huang, 2016b; Pan et al., 2018b), these approaches assumed constant muscle moment arm profiles. Furthermore, Gonzalez (Gonzalez et al., 1997) showed that the maximum torque production at a joint was dependent on posture, which contradicts constant moment arm relationships. Even though the constant moment arm simplification has its drawbacks, its benefit is simplifying complex muscle-DOF relationships. While dynamic moment 
arms can be more physiologically accurate, these moment arms should not be used without a validation process like the one described in Chapter 2. Without appropriate validation, dynamic moment arms could potentially lead to inconsistencies where the constant moment arm assumption would not.

Future development will test if the model accurately represents the general population or if it requires subject-specific scaling. If a generic model can adequately describe the musculoskeletal dynamics without subject-specific scaling, then it would greatly simplify its implementation in many applications. Additionally, the data from our model can be accessed in real-time (Sobinov et al., 2019) applications, which provides a valuable resource for humanmachine interfaces. An example would use the live muscle approximations to monitor an individual's movements to avoid muscle injury or control a prosthesis. Future studies will validate additional body segments in order to move towards a full-body musculoskeletal description.

\subsection{Hand Prosthetic Control}

Controlling a prosthetic hand with a musculoskeletal model has been previously shown (Pan et al., 2018b). In that work, they only had 2 movements in their model, which were wrist flexion/extension and finger MCP flexion/extension, while we showed control of an 18 DOF hand (See Supplementary Table 3-1 for simulated DOFs) that has natural physiological couplings to make 8 independent movements. The 8 movements were as follows: index through pinky finger flexion/extension, thumb flexion/extension, thumb abduction/adduction, wrist flexion/extension, and wrist pronation/supination. Pan et al. (Pan et al., 2018b) also simplified their musculoskeletal dynamics by assuming a constant moment arm for each muscle-DOF relationship. In our model, we chose to keep the posture dependent moment arms to maintain physiological accuracy. Moment arms are posture dependent and slight differences in them can 
amount to a large change in torque that a muscle can produce (Gonzalez et al., 1997). In chapter 2, we showed how there is a significant difference in the moment arms at the wrist through the range of motion, which amounts to the same force creating significantly different torques based on the posture. Making the torques generated change based on the posture helps meet the overall goal of making the prosthesis controller as intuitive as possible.

Machine learning is another method that attempts to create an intuitive prosthetic controller. An EMG classifier is a standard machine learning approach for controlling a myoelectric prosthesis. This process requires training data to be collected for each recalibration of the system (Birdwell et al., 2015; Smith et al., 2014). The main issue with the training dataset is ensuring that there is enough training data for the classifier to identify the desired movement. This problem is not trivial because people can perform the same movement in a variety of ways and postures. This is not a problem for our biomimetic approach because it models the posture of the hand and applies muscle forces about that posture. This process allows the appropriate torques to be applied no matter the posture of the arm.

The controllable DOFs on a prosthesis can vary widely from a 1 DOF gripper (i.e., open/close) to a 6 DOF hand (e.g., luke hand (Resnik et al., 2018a)) and in the future potentially even more. Prosthetic control approaches need to keep this idea in mind so that their controller is not limited to only one type of prosthesis. Our approach, described in chapter 3, allows us to control any prosthesis with reasonable anthropomorphic correspondence between physiological and prosthetic DOFs. There is a limit to the number of independent movements that we can expect to achieve with our model. At most, our method can currently achieve 8 independent movements: index through pinky finger flexion/extension, thumb flexion/extension, thumb abduction/adduction, wrist flexion/extension, and wrist pronation/supination. It requires at least 1 agonist-antagonist muscle pair per movement for complete independence. For example, to control wrist supination/pronation, it would require a wrist supination muscle and a wrist 
pronation muscle. To have all 8 movements be independent, it would require recording from 16 muscles, at least. The issue is that some of the muscles are close together and can be hard to get individual sensors on the correct muscles. For surface EMG sensors, there is also the issue of cross-talk where one sensor could record data from multiple muscles at once. Intramuscular EMG sensors can mitigate the issue of cross-talk but are challenging to place in the correct muscle.

Future work will investigate the potential to create a transformation between processed EMG and simplified control signals. The goal is to remove the inherent noise found in EMG by going from processed EMG to simplified muscle activations. We started the first step in this process by creating optimized Gaussian signals that could generate simulated hand movements. While these signals could simulate some actions, it was not possible to perform movements that needed to maintain the wrist posture. Nonetheless, we generated control patterns composed of single Gaussians to represent the coordinated activation patterns for all simulated muscles. The spatial and temporal characteristics of each Gaussian were optimized to improve movement performance. To our surprise, the artificial patterns were correlated with experimental EMG, as indicated by Fig. 3-6. This result was encouraging because a transformation could be possible once all the movements can be completed. Most likely, single Gaussians per muscle are not complex enough to produce patterns that can maintain wrist posture. The inclusion of two Gaussians for each signal could ideally address the issue and allow more complex burst patterns to be created.

\subsection{Gait Rehabilitation}

Thus far, we have discussed applying a biomimetic approach to prosthetic hand control, and now we will switch to discussing a biomimetic approach for the lower limb. We can use our understanding of the human body and implement it for the development and improvement of 
rehabilitation techniques such as gait rehabilitation. Current technologies in gait rehabilitation could benefit from more modern technology (Mikolajczyk et al., 2018), and one that is beginning to be adopted is split-belt treadmill training (Finley et al., 2015), which is the system we used to create a self-paced treadmill.

The goal of a self-paced treadmill is to allow the user to change their speed and minimize the number of unnecessary accelerations and decelerations, so the walking feels natural. We approached the development of a self-paced treadmill keeping in mind how the biological system allows us to walk. The central nervous system contains spinal neural mechanisms called central pattern generators (CPGs) that are responsible for spinal rhythmogenesis (Grillner, 1985). This neural structure is a target of both feedforward and feedback control pathways involved in the initiation and regulation of gait. In our laboratory, we have developed data-driven CPG models (Yakovenko et al., 2018) that predict the nature of at least some of the main inputs, which happens to be limb speed. This idea inspired us to create a self-paced treadmill that worked like an inverse spinal CPG that used a legs stance time, swing time, and previous speed to calculate the current speed. We only changed the treadmill belt speeds once per step, but the drawback is that when a person changes their speed on the treadmill, they move forward or backward before the treadmill can adjust.

The typical approach to a self-paced treadmill is based on position and adjusting the treadmill speed to keep the person in the middle. The hardware used to record the participant's position is usually either a depth sensor (Jonghyun Kim et al., 2013; Minetti et al., 2003) or motion capture (Sloot et al., 2014b; Souman et al., 2011; Stavar et al., 2011). Our self-paced treadmill also involves keeping the person in the middle of the treadmill (see 4.4.5 Speed Adjustment), but there are two significant differences from the other systems. The first difference is that we calculate each limb speed independently, allowing us to simulate step turning (see Fig. 4-6), while the other systems only calculate a whole body speed. The difference here is important 
because it has been shown that walking straight forward does not involve the motor cortex as much as more challenging tasks like stepping over obstacles (Drew et al., 2008) or turning. The aim of rehabilitation is to improve motor function through the mechanisms of neuroplasticity; therefore, rehabilitative tasks which are designed to necessitate the involvement of the motor cortex may facilitate motor recovery. The second difference between our system and others is that our system works based on calculating leg stance and swing times from ground reaction forces. While motion capture data could be used to calculate step phase times, without it there is no additional setup time required applying hardware to the participant walking. Our quick, minimal setup, therefore, makes our system more viable for clinical adoption. Our system also provides visual feedback to the person walking, which can be used by clinicians to give feedback and tasks to participants.

Self-paced treadmills enable people to adjust their speed, more closely mirroring the action of walking over-ground. (Plotnik et al., 2015; Sloot et al., 2014b). Adding visual feedback to treadmill walking has been shown to improve gait parameters (e.g., stride length) to more closely match over-ground walking (Sloot et al., 2014a). Furthermore, turning strategies have been observed in people walking on a treadmill if they are provided coherent visual feedback (Oh et al., 2018). Our system calculates leg speeds independently, which allows differences in limb speeds to be interpreted into visual flow heading direction, i.e., turning. This capability on a self-paced treadmill could be a significant advancement for gait rehabilitation. It has been shown in animals that walking straight does not have an increase in the motor cortex firing (Drew et al., 2008). Specifically, recordings of pyramidal tract neurons, from the motor cortex, do not show an increase in discharge frequency in cats walking in a straight trajectory (Drew et al., 2008). By adding turning to split-belt treadmill walking, we can require motor planning, which can better involve the motor cortex and potentially lead to better recovery. With the Hebbian principle of "what fires together wires together" (Hebb, 1949) in mind, having people walk and perform 
turning actions on a split-belt treadmill with visual flow provides a platform for studying and improving gait rehabilitation.

Matching the visual flow to the user's actions closes the loop on the robotic system and places the human in the control loop. Future work can use this system to investigate the internal mechanisms used for locomotion. Current studies have shown that performing treadmill gait adaptations while blindfolded helped reinforce the adaptations carry over to natural over-ground walking (Torres-Oviedo et al., 2011; Torres-Oviedo and Bastian, 2010). The self-paced treadmill system could be used to test if gait adaptation retention improves when the participant has visual flow that matches the walking adaptation. Another interesting study would be to have people with an asymmetric gait attempt to walk more symmetrically based on feedback from the self-paced treadmill system. Then see if there is a learned behavior and if that behavior would carry over to over-ground walking. Future work will show if our self-paced treadmill system can be used in gait adaptation rehabilitation.

\subsection{Limitations}

There are several limitations to the studies described in the dissertation. In chapter 2, the limitation was in relation to the sparse dataset used to compare simulated and published data. Hand and arm muscles span on average 3 DOFs, but often data is only published for one DOF while the other DOFs are held constant (i.e., same posture). We were forced to create validation datasets that were combined from available literature and contained inter-subject and measurement techniques (i.e., cadaver or magnetic resonance imaging) variability. These discrepancies have the potential to cause inconsistencies in the muscle parameters (see 2.4.2 Datasets). We mitigated these inconsistencies with the developed validation tools (see Section 2.4.3.2). In chapter 3 , the main limitation was correctly identifying the muscle data being recorded. We identified the recorded muscles by observing their patterns in a diverse set of 
movements. EMG cross-talk and the co-contraction of antagonistic muscle groups result in the correlated activity of muscles, which impede the identification. Future work would involve creating a system capable of deciphering the muscles being recorded. In chapter 4 , the main limitation in the self-paced treadmill work is that it requires one full step before a calculation occurs. This effectively makes the treadmill one step behind the user, so future work would be to include another speed calculation that would help predict speed changed. Future work will involve adding another speed measurement to the leg speed calculation based on a feedforward approach using the ground reaction forces.

\subsection{Conclusion}

In my dissertation, I was able to use a biomimetic approach to target two different applications in restorative robotics, one involving the upper limb and one involving the lower limb. For upper limb control, we developed and validated a musculoskeletal model of the forearm and hand and used it to control different upper limb prostheses. Our grouped signal processing was able to provide signals to muscles that were not recorded, which allowed us to decipher movement intent. This deciphering of movement intent in real-time with musculoskeletal models can be utilized to create a new generation of prosthetic controllers. For lower limb control, we developed a self-paced treadmill that allows for realistic omnidirectional walking in virtual environments that can be used as a tool to investigate human locomotion and improve gait rehabilitation. Through these two systems, it shows how a biomimetic approach can be applied broadly to help improve the field of restorative robotics. 


\section{References}

Ackermann M, Schiehlen W. 2006. Dynamic Analysis of Human Gait Disorder and Metabolical Cost Estimation. Arch Appl Mech 75:569-594. doi:10.1007/s00419-006-0027-7

Akram SB, Frank JS, Chenouri S. 2010. Turning behavior in healthy older adults: Is there a preference for step versus spin turns? Gait Posture 31:23-6.

doi:10.1016/j.gaitpost.2009.08.238

Alton F, Baldey L, Caplan S, Morrissey MC. 1998. A kinematic comparison of overground and treadmill walking. Clin Biomech 13:434-440. doi:10.1016/S0268-0033(98)00012-6

Amis AA, Dowson D, Wright V. 1979. Muscle Strengths and Musculoskeletal Geometry of the Upper Limb. Eng Med 8:41-48. doi:10.1243/EMED_JOUR_1979_008_010_02

An KN, Hui FC, Morrey BF, Linscheid RL, Chao EY. 1981. Muscles across the elbow joint: A biomechanical analysis. J Biomech 14:659-669. doi:10.1016/0021-9290(81)90048-8

Arkin AM. 1941. Absolute muscle power. Arch Surg 42:395. doi:10.1001/archsurg.1941.01210080195012

Arnold AS, Blemker SS, Delp SL. 2001. Evaluation of a deformable musculoskeletal model for estimating muscle-tendon lengths during crouch gait. Ann Biomed Eng 29:263-74.

Arnold AS, Salinas S, Asakawa DJ, Delp SL. 2000. Accuracy of muscle moment arms estimated from MRI-based musculoskeletal models of the lower extremity. Comput Aided Surg 5:108-119. doi:10.1002/1097-0150(2000)5:2<108::AID-IGS5>3.0.CO;2-2

Arnold EM, Ward SR, Lieber RL, Delp SL. 2010. A Model of the Lower Limb for Analysis of Human Movement. Ann Biomed Eng 38:269-279. doi:10.1007/s10439-009-9852-5

Au S, Berniker M, Herr H. 2008. Powered ankle-foot prosthesis to assist level-ground and stairdescent gaits. Neural Networks 21:654-666. doi:10.1016/j.neunet.2008.03.006

Au SK, Herr H, Weber J, Martinez-Villalpando EC. 2007. Powered Ankle-Foot Prosthesis for the Improvement of Amputee Ambulation2007 29th Annual International Conference of the IEEE Engineering in Medicine and Biology Society. IEEE. pp. 3020-3026.

doi:10.1109/IEMBS.2007.4352965

Berke GM, Fergason J, Milani JR, Hattingh J, McDowell M, Nguyen V, Reiber GE. 2010. Comparison of satisfaction with current prosthetic care in veterans and servicemembers from Vietnam and OIF/OEF conflicts with major traumatic limb loss. J Rehabil Res Dev 47:361-71.

Bernstein N. 1967. The Co-ordination and Regulation of Movements, Journal of Neuropathology and Experimental Neurology. Oxford: Pergamon Press.

Biddiss EA, Chau TT. 2007. Upper limb prosthesis use and abandonment: A survey of the last 25 years. Prosthet Orthot Int 31:236-257. doi:10.1080/03093640600994581

Birdwell JA, Hargrove LJ, Weir RF ff, Kuiken TA. 2015. Extrinsic Finger and Thumb Muscles Command a Virtual Hand to Allow Individual Finger and Grasp Control. IEEE Trans Biomed Eng 62:218-226. doi:10.1109/TBME.2014.2344854 
Bourbonnais D, Duval P. 1991. Static dynamometer for the measurement of multidirectional forces exerted by the thumb. Med Biol Eng Comput 29:413-418. doi:10.1007/BF02441663

Box GEP. 1979. Robustness in the Strategy of Scientific Model BuildingRobustness in Statistics. Elsevier. pp. 201-236. doi:10.1016/B978-0-12-438150-6.50018-2

Brand PW, Beach RB, Thompson DE. 1981. Relative tension and potential excursion of muscles in the forearm and hand. $J$ Hand Surg Am 6:209-219. doi:10.1016/S03635023(81)80072-X

Bremer AK, Sennwald GR, Favre P, Jacob HAC. 2006. Moment arms of forearm rotators. Clin Biomech 21:683-691. doi:10.1016/j.clinbiomech.2006.03.002

Brown TG. 1914. On the nature of the fundamental activity of the nervous centres; together with an analysis of the conditioning of rhythmic activity in progression, and a theory of the evolution of function in the nervous system. J Physiol 48:18-46.

doi:10.1113/jphysiol.1914.sp001646

Brown TG. 1911. The intrinsic factors in the act of progression in the mammal. Proc $R$ Soc London 84:308-319.

Buchanan TS. 1995. Evidence that maximum muscle stress is not a constant: differences in specific tension in elbow flexors and extensors. Med Eng Phys 17:529-36. doi:10.1016/1350-4533(95)00005-8

Carbone V, Fluit R, Pellikaan P, van der Krogt MM, Janssen D, Damsgaard M, Vigneron L, Feilkas T, Koopman HFJM, Verdonschot N. 2015. TLEM 2.0 - A comprehensive musculoskeletal geometry dataset for subject-specific modeling of lower extremity. $J$ Biomech 48:734-741. doi:10.1016/j.jbiomech.2014.12.034

Carhart M, He J, Herman R, D'Luzansky S, Willis W. 2004. Epidural Spinal-Cord Stimulation Facilitates Recovery of Functional Walking Following Incomplete Spinal-Cord Injury. IEEE Trans Neural Syst Rehabil Eng 12:32-42. doi:10.1109/TNSRE.2003.822763

Carrozza MC, Cappiello G, Micera S, Edin BB, Beccai L, Cipriani C. 2006. Design of a cybernetic hand for perception and action. Biol Cybern 95:629-644. doi:10.1007/s00422006-0124-2

Chao EYS, An K-N, Cooney WP, Linscheid RL. 1989. Biomechanics of the Hand. WORLD SCIENTIFIC. doi:10.1142/0321

Churchland MM, Cunningham JP, Kaufman MT, Foster JD, Nuyujukian P, Ryu SI, Shenoy K V. 2012. Neural population dynamics during reaching. Nature 487:51-56.

doi:10.1038/nature11129

Clauser CE, Young JW, McConville JT. 1969. Weight, Volume, and Center of Mass of Segments of the Human Body. Natl Tech Inf Serv 1-112.

Collins JJ, Richmond SA. 1994. Hard-wired central pattern generators for quadrupedal locomotion. Biol Cybern 71:375-385. doi:10.1007/BF00198915

Cordella F, Ciancio AL, Sacchetti R, Davalli A, Cutti AG, Guglielmelli E, Zollo L. 2016. Literature Review on Needs of Upper Limb Prosthesis Users. Front Neurosci 10:1-14. doi:10.3389/fnins.2016.00209

Courtine G, Papaxanthis C, Schieppati M. 2006. Coordinated modulation of locomotor muscle 
synergies constructs straight-ahead and curvilinear walking in humans. Exp Brain Res 170:320-335. doi:10.1007/s00221-005-0215-7

Crouch D, Huang H. 2016a. Lumped-parameter electromyogram-driven musculoskeletal hand model: A potential platform for real-time prosthesis control. J Biomech 49:3901-3907. doi:10.1016/j.jbiomech.2016.10.035

Crouch D, Huang H. 2016b. Simple EMG-driven musculoskeletal model enables consistent control performance during path tracing tasks2016 38th Annual International Conference of the IEEE Engineering in Medicine and Biology Society (EMBC). IEEE. pp. 1-4. doi:10.1109/EMBC.2016.7590625

Crouch D, Pan L, Filer W, Stallings JW, Huang H. 2018. Comparing Surface and Intramuscular Electromyography for Simultaneous and Proportional Control Based on a Musculoskeletal Model: A Pilot Study. IEEE Trans Neural Syst Rehabil Eng 26:1735-1744. doi:10.1109/TNSRE.2018.2859833

Dantas H, Warren DJ, Wendelken S, Davis T, Clark GA, Mathews VJ. 2019. Deep Learning Movement Intent Decoders Trained with Dataset Aggregation for Prosthetic Limb Control. IEEE Trans Biomed Eng PP:1-1. doi:10.1109/TBME.2019.2901882

De Luca CJ, Donald Gilmore L, Kuznetsov M, Roy SH. 2010. Filtering the surface EMG signal: Movement artifact and baseline noise contamination. J Biomech 43:1573-1579. doi:10.1016/j.jbiomech.2010.01.027

de Zee M, Dalstra M, Cattaneo PM, Rasmussen J, Svensson P, Melsen B. 2007. Validation of a musculo-skeletal model of the mandible and its application to mandibular distraction osteogenesis. J Biomech 40:1192-1201. doi:10.1016/j.jbiomech.2006.06.024

Decostre V, Canal A, Ollivier G, Ledoux I, Moraux A, Doppler V, Payan CAM, Hogrel J-Y. 2015. Wrist flexion and extension torques measured by highly sensitive dynamometer in healthy subjects from 5 to 80 years. BMC Musculoskelet Disord 16:4. doi:10.1186/s12891-0150458-9

Delp SL, Anderson FC, Arnold AS, Loan P, Habib A, John CT, Guendelman E, Thelen DG. 2007. OpenSim: Open source to create and analyze dynamic simulations of movement. IEEE Trans Biomed Eng 54:1940-1950. doi:10.1109/TBME.2007.901024

Delp SL, Loan JP, Hoy MG, Zajac FE, Topp EL, Rosen JM. 1990. An interactive graphics-based model of the lower extremity to study orthopaedic surgical procedures. IEEE Trans Biomed Eng 37:757-767. doi:10.1109/10.102791

Domkin D, Laczko J, Jaric S, Johansson H, Latash ML. 2002. Structure of joint variability in bimanual pointing tasks. Exp Brain Res 143:11-23. doi:10.1007/s00221-001-0944-1

Drew T, Andujar J-E, Lajoie K, Yakovenko S. 2008. Cortical mechanisms involved in visuomotor coordination during precision walking. Brain Res Rev 57:199-211. doi:10.1016/j.brainresrev.2007.07.017

Edgerton VR, Apor P, Roy RR. 1990. Specific tension of human elbow flexor muscles. Acta Physiol Hung 75:205-16.

Farina D, Negro F, Gazzoni M, Enoka RM. 2008. Detecting the Unique Representation of Motor-Unit Action Potentials in the Surface Electromyogram. J Neurophysiol 100:12231233. doi:10.1152/jn.90219.2008 
Finley JM, Long A, Bastian AJ, Torres-Oviedo G. 2015. Spatial and Temporal Control Contribute to Step Length Asymmetry During Split-Belt Adaptation and Hemiparetic Gait. Neurorehabil Neural Repair 29:786-795. doi:10.1177/1545968314567149

Fisher RA. 1925. Statistical Methods For Research Workers. Genesis Publishing Pvt Ltd.

Fowler N., Nicol A., Condon B, Hadley D. 2001. Method of determination of three dimensional index finger moment arms and tendon lines of action using high resolution MRI scans. $J$ Biomech 34:791-797. doi:10.1016/S0021-9290(01)00021-5

Fowlkes EB, Mallows CL. 1983. A Method for Comparing Two Hierarchical Clusterings. J Am Stat Assoc 78:553. doi:10.2307/2288117

Gillard DM, Yakovenko S, Cameron T, Prochazka A. 2000. Isometric muscle length-tension curves do not predict angle-torque curves of human wrist in continuous active movements. J Biomech 33:1341-8. doi:10.1016/s0021-9290(00)00127-5

Goislard De Monsabert B, Edwards D, Shah D, Kedgley A. 2018. Importance of Consistent Datasets in Musculoskeletal Modelling: A Study of the Hand and Wrist. Ann Biomed Eng 46:71-85. doi:10.1007/s10439-017-1936-z

Gonzalez R V, Buchanan TS, Delp SL. 1997. How muscle architecture and moment arms affect wrist flexion-extension moments. J Biomech 30:705-712. doi:10.1016/S00219290(97)00015-8

Gordon KD, Pardo RD, Johnson JA, King GJW, Miller TA. 2004. Electromyographic activity and strength during maximum isometric pronation and supination efforts in healthy adults. $J$ Orthop Res 22:208-13. doi:10.1016/S0736-0266(03)00115-3

Graczyk EL, Resnik L, Schiefer MA, Schmitt MS, Tyler DJ. 2018. Home Use of a Neuralconnected Sensory Prosthesis Provides the Functional and Psychosocial Experience of Having a Hand Again. Sci Rep 8:9866. doi:10.1038/s41598-018-26952-x

Graupe D, Beex AA, Monlux WJ, Magnussen I. 1977. A multifunctional prosthesis control system based on time series identification of EMG signals using microprocessors. Bull Prosthet Res 10:4-16.

Grillner S. 2006. Biological Pattern Generation: The Cellular and Computational Logic of Networks in Motion. Neuron 52:751-766. doi:10.1016/j.neuron.2006.11.008

Grillner S. 1985. Neurobiological bases of rhythmic motor acts in vertebrates. Science (80-) 228:143-149. doi:10.1126/science.3975635

Grillner S. 1981. Control of Locomotion in Bipeds, Tetrapods, and Fish In: Brookhart J, Mountcastle V, editors. Handbook of Physiology, The Nervous System, Motor Control. pp. 1179-1236.

Grillner S, Perret C, Zangger P. 1976. Central generation of locomotion in the spinal dogfish. Brain Res 109:255-69. doi:10.1016/0006-8993(76)90529-1

Grillner S, Zangger P. 1975. How detailed is the central pattern generation for locomotion? Brain Res 88:367-371. doi:10.1016/0006-8993(75)90401-1

Grimmer M, Holgate M, Holgate R, Boehler A, Ward J, Hollander K, Sugar T, Seyfarth A. 2016. A powered prosthetic ankle joint for walking and running. Biomed Eng Online 15:141. doi:10.1186/s12938-016-0286-7 
Gritsenko V, Hardesty RL, Boots MT, Yakovenko S. 2016. Biomechanical Constraints Underlying Motor Primitives Derived from the Musculoskeletal Anatomy of the Human Arm. PLoS One 11:e0164050. doi:10.1371/journal.pone.0164050

Halbertsma JM. 1983. The stride cycle of the cat: the modelling of locomotion by computerized analysis of automatic recordings. Acta Physiol Scand Suppl 521:1-75.

Hamner SR, Seth A, Delp SL. 2010. Muscle contributions to propulsion and support during running. J Biomech 43:2709-2716. doi:10.1016/j.jbiomech.2010.06.025

Happee R, Van der Helm FCT. 1995. The control of shoulder muscles during goal directed movements, an inverse dynamic analysis. J Biomech 28:1179-1191. doi:10.1016/00219290(94)00181-3

Hase K, Stein RB. 1999. Turning strategies during human walking. J Neurophysiol 81:2914-22. doi:10.1152/jn.1999.81.6.2914

Haugstvedt JR, Berger R a, Berglund LJ. 2001. A mechanical study of the moment-forces of the supinators and pronators of the forearm. Acta Orthop Scand 72:629-634. doi:10.1080/000164701317269076

Haxton HA. 1944. Absolute muscle force in the ankle flexors of man. $J$ Physiol 103:267-273. doi:10.1113/jphysiol.1944.sp004075

Hebb DO. 1949. Organization of Behavior: A Neuropsychological Theory.

Henninger HB, Reese SP, Anderson AE, Weiss JA. 2010. Validation of computational models in biomechanics. Proc Inst Mech Eng H 224:801-12. doi:10.1243/09544119JEIM649

Hill A. 1938. The heat of shortening and the dynamic constants of muscle. Proc $R$ Soc London Ser B - Biol Sci 126:136-195. doi:10.1098/rspb.1938.0050

Holzbaur KRS, Murray WM, Delp SL. 2005. A Model of the Upper Extremity for Simulating Musculoskeletal Surgery and Analyzing Neuromuscular Control. Ann Biomed Eng 33:829840. doi:10.1007/s10439-005-3320-7

Horsman MK. 2007. The Twente Lower Extremity Model: Consistent Dynamic Simulation of the Human Locomotor Apparatus.

Ison M, Artemiadis P. 2014. The role of muscle synergies in myoelectric control: trends and challenges for simultaneous multifunction control. J Neural Eng 11:051001. doi:10.1088/1741-2560/11/5/051001

Ivanenko YP, Grasso R, Lacquaniti F. 2000. Influence of Leg Muscle Vibration on Human Walking. J Neurophysiol 84:1737-1747. doi:10.1152/jn.2000.84.4.1737

Ivanenko YP, Poppele RE, Lacquaniti F. 2004. Five basic muscle activation patterns account for muscle activity during human locomotion. J Physiol 556:267-282.

doi:10.1113/jphysiol.2003.057174

Jonghyun Kim, Gravunder A, Stanley CJ, Hyung-Soon Park. 2013. Low-cost implementation of a self-paced treadmill by using a commercial depth sensor2013 35th Annual International Conference of the IEEE Engineering in Medicine and Biology Society (EMBC). IEEE. pp. 874-877. doi:10.1109/EMBC.2013.6609640

Kiehn O. 2006. Locomotor circuits in the mammalian spinal cord. Annu Rev Neurosci 29:279306. doi:10.1146/annurev.neuro.29.051605.112910 
Kirchner JW. 2006. Getting the right answers for the right reasons: Linking measurements, analyses, and models to advance the science of hydrology. Water Resour Res 42:1-5. doi:10.1029/2005WR004362

Koh S, Buford WL, Andersen CR, Viegas SF. 2006. Intrinsic Muscle Contribution to the Metacarpophalangeal Joint Flexion Moment of the Middle, Ring, and Small Fingers. $J$ Hand Surg Am 31:1111-1117. doi:10.1016/j.jhsa.2006.03.003

Kumar V, Todorov E. 2015. MuJoCo HAPTIX: A virtual reality system for hand manipulation2015 IEEE-RAS 15th International Conference on Humanoid Robots (Humanoids). IEEE. pp. 657-663. doi:10.1109/HUMANOIDS.2015.7363441

Kyberd PJ, Light C, Chappell PH, Nightingale JM, Whatley D, Evans M. 2001. The design of anthropomorphic prosthetic hands: A study of the Southampton Hand. Robotica 19:593600. doi:10.1017/S0263574701003538

Lambert-Shirzad N, Van der Loos HFM. 2017. On identifying kinematic and muscle synergies: a comparison of matrix factorization methods using experimental data from the healthy population. J Neurophysiol 117:290-302. doi:10.1152/jn.00435.2016

Laurent K, De Sèze M-P, Delleci C, Koleck M, Dehail P, Orgogozo J-M, Mazaux J-M. 2011. Assessment of quality of life in stroke patients with hemiplegia. Ann Phys Rehabil Med 54:376-390. doi:10.1016/j.rehab.2011.06.002

Lee SJ, Hidler J. 2008. Biomechanics of overground vs. treadmill walking in healthy individuals. J Appl Physiol 104:747-755. doi:10.1152/japplphysiol.01380.2006

Lichtenstein L, Barabas J, Woods RL, Peli E. 2007. A feedback-controlled interface for treadmill locomotion in virtual environments. ACM Trans Appl Percept 4:7-es. doi:10.1145/1227134.1227141

Lillicrap TP, Scott SH. 2013. Preference Distributions of Primary Motor Cortex Neurons Reflect Control Solutions Optimized for Limb Biomechanics. Neuron 77:168-179.

doi:10.1016/j.neuron.2012.10.041

Loren GJ, Shoemaker SD, Burkholder TJ, Jacobson MD, Fridén J, Lieber RL. 1996. Human wrist motors: biomechanical design and application to tendon transfers. J Biomech 29:33142. doi:10.1016/0021-9290(95)00055-0

Lund ME, de Zee M, Andersen MS, Rasmussen J. 2012. On validation of multibody musculoskeletal models. Proc Inst Mech Eng Part H J Eng Med 226:82-94. doi:10.1177/0954411911431516

Lundberg A, Phillips CG. 1973. T. Graham Brown's film on locomotion in the decerebrate cat. J Physiol 231:90-91.

McCrea DA, Rybak IA. 2008. Organization of mammalian locomotor rhythm and pattern generation. Brain Res Rev 57:134-46. doi:10.1016/j.brainresrev.2007.08.006

Meehan CF, Grondahl L, Nielsen JB, Hultborn H. 2012. Fictive locomotion in the adult decerebrate and spinal mouse in vivo. J Physiol 590:289-300. doi:10.1113/jphysiol.2011.214643

Menegaldo LL, de Toledo Fleury A, Weber HI. 2004. Moment arms and musculotendon lengths estimation for a three-dimensional lower-limb model. J Biomech 37:1447-1453.

doi:10.1016/j.jbiomech.2003.12.017 
Mikolajczyk T, Ciobanu I, Badea DI, Iliescu A, Pizzamiglio S, Schauer T, Seel T, Seiciu PL, Turner DL, Berteanu M. 2018. Advanced technology for gait rehabilitation: An overview. Adv Mech Eng 10:168781401878362. doi:10.1177/1687814018783627

Miller LC, Thompson CK, Negro F, Heckman CJ, Farina D, Dewald JPA. 2014. High-density surface EMG decomposition allows for recording of motor unit discharge from proximal and distal flexion synergy muscles simultaneously in individuals with stroke2014 36th Annual International Conference of the IEEE Engineering in Medicine and Biology Society. IEEE. pp. 5340-5344. doi:10.1109/EMBC.2014.6944832

Minetti AE, Boldrini L, Brusamolin L, Zamparo P, McKee T. 2003. A feedback-controlled treadmill (treadmill-on-demand) and the spontaneous speed of walking and running in humans. J Appl Physiol 95:838-843. doi:10.1152/japplphysiol.00128.2003

Murray WM, Delp SL, Buchanan TS. 1995. Variation of muscle moment arms with elbow and forearm position. J Biomech 28:513-25. doi:10.1016/0021-9290(94)00114-j

Narici M V., Roi GS, Landoni L. 1988. Force of knee extensor and flexor muscles and crosssectional area determined by nuclear magnetic resonance imaging. Eur J Appl Physiol Occup Physiol 57:39-44. doi:10.1007/bf00691235

Netter FH. 2011. Atlas of Human Anatomy 5th Edition, Netter Basic Science.

Nussbaum MA, Chaffin DB, Rechtien CJ. 1995. Muscle lines-of-action affect predicted forces in optimization-based spine muscle modeling. J Biomech 28:401-9. doi:10.1016/00219290(94)00078-i

Oh K, Stanley CJ, Damiano DL, Kim J, Yoon J, Park H-S. 2018. Biomechanical evaluation of virtual reality-based turning on a self-paced linear treadmill. Gait Posture 65:157-162. doi:10.1016/j.gaitpost.2018.07.175

Okoro CA, Hollis ND, Cyrus AC, Griffin-Blake S. 2018. Prevalence of Disabilities and Health Care Access by Disability Status and Type Among Adults — United States, 2016. MMWR Morb Mortal Wkly Rep 67:882-887. doi:10.15585/mmwr.mm6732a3

Pan L, Crouch D, Huang H. 2018a. Myoelectric Control Based on a Generic Musculoskeletal Model: Toward a Multi-User Neural-Machine Interface. IEEE Trans Neural Syst Rehabil Eng 26:1435-1442. doi:10.1109/TNSRE.2018.2838448

Pan L, Harmody A, Huang H. 2018b. A Reliable Multi-User EMG Interface Based on A GenericMusculoskeletal Model against Loading Weight Changes*2018 40th Annual International Conference of the IEEE Engineering in Medicine and Biology Society (EMBC). IEEE. pp. 2104-2107. doi:10.1109/EMBC.2018.8512685

Patla AE, Calvert TW, Stein RB. 1985. Model of a pattern generator for locomotion in mammals. Am J Physiol Integr Comp Physiol 248:R484-R494. doi:10.1152/ajpregu.1985.248.4.R484

Pfeifer R, Lungarella M, lida F. 2007. Self-Organization, Embodiment, and Biologically Inspired Robotics. Science (80- ) 318:1088-1093. doi:10.1126/science.1145803

Pinzur MS, Angelats J, Light TR, Izuierdo R, Pluth T. 1994. Functional outcome following traumatic upper limb amputation and prosthetic limb fitting. J Hand Surg Am 19:836-839. doi:10.1016/0363-5023(94)90197-X

Plotnik M, Azrad T, Bondi M, Bahat Y, Gimmon Y, Zeilig G, Inzelberg R, Siev-Ner I. 2015. Selfselected gait speed - over ground versus self-paced treadmill walking, a solution for a 
paradox. J Neuroeng Rehabil 12:20. doi:10.1186/s12984-015-0002-z

Popov A, Yakovenko S. 2015. Muscle synergy decomposition analysis using wavelet detection in human locomotor activity2015 Signal Processing Symposium (SPSympo). IEEE. pp. 15. doi:10.1109/SPS.2015.7168260

Raichle KA, Hanley MA, Molton I, Kadel NJ, Campbell K, Phelps E, Ehde D, Smith DG. 2008. Prosthesis use in persons with lower- and upper-limb amputation. J Rehabil Res Dev 45:961-72.

Rajagopal A, Dembia CL, DeMers MS, Delp DD, Hicks JL, Delp SL. 2016. Full-Body Musculoskeletal Model for Muscle-Driven Simulation of Human Gait. IEEE Trans Biomed Eng 63:2068-2079. doi:10.1109/TBME.2016.2586891

Resnik L, Acluche F, Borgia M. 2018a. The DEKA hand: A multifunction prosthetic terminal device-patterns of grip usage at home. Prosthet Orthot Int 42:446-454. doi:10.1177/0309364617728117

Resnik L, Huang H, Winslow A, Crouch DL, Zhang F, Wolk N. 2018b. Evaluation of EMG pattern recognition for upper limb prosthesis control: a case study in comparison with direct myoelectric control. J Neuroeng Rehabil 15:23. doi:10.1186/s12984-018-0361-3

Riley PO, Paolini G, Della Croce U, Paylo KW, Kerrigan DC. 2007. A kinematic and kinetic comparison of overground and treadmill walking in healthy subjects. Gait Posture 26:1724. doi:10.1016/j.gaitpost.2006.07.003

Rohatgi A. 2018. WebPlotDigitizer. https://automeris.io/WebPlotDigitizer

Romero F, Alonso FJ. 2016. A comparison among different Hill-type contraction dynamics formulations for muscle force estimation. Mech Sci 7:19-29. doi:10.5194/ms-7-19-2016

Rybak IA, Dougherty KJ, Shevtsova NA. 2015. Organization of the Mammalian Locomotor CPG: Review of Computational Model and Circuit Architectures Based on Genetically Identified Spinal Interneurons. eneuro 2:ENEURO.0069-15.2015. doi:10.1523/ENEURO.006915.2015

Rybak IA, Shevtsova NA, Lafreniere-Roula M, McCrea DA. 2006. Modelling spinal circuitry involved in locomotor pattern generation: insights from deletions during fictive locomotion. $J$ Physiol 577:617-639. doi:10.1113/jphysiol.2006.118703

Sartori M, Durandau G, Došen S, Farina D. 2018. Robust simultaneous myoelectric control of multiple degrees of freedom in wrist-hand prostheses by real-time neuromusculoskeletal modeling. J Neural Eng 15:066026. doi:10.1088/1741-2552/aae26b

Sartori M, Reggiani M, van den Bogert AJ, Lloyd DG. 2012. Estimation of musculotendon kinematics in large musculoskeletal models using multidimensional B-splines. J Biomech 45:595-601. doi:10.1016/j.jbiomech.2011.10.040

Saul KR, Hu X, Goehler CM, Vidt ME, Daly M, Velisar A, Murray WM. 2015a. Benchmarking of dynamic simulation predictions in two software platforms using an upper limb musculoskeletal model. Comput Methods Biomech Biomed Engin 18:1445-1458. doi:10.1080/10255842.2014.916698

Saul KR, Vidt ME, Gold GE, Murray WM. 2015b. Upper Limb Strength and Muscle Volume in Healthy Middle-Aged Adults. J Appl Biomech 31:484-491. doi:10.1123/jab.2014-0177 
Scheme E, Englehart K. 2011. Electromyogram pattern recognition for control of powered upper-limb prostheses: State of the art and challenges for clinical use. J Rehabil Res Dev 48:643. doi:10.1682/JRRD.2010.09.0177

Scheme E, Lock B, Hargrove L, Hill W, Kuruganti U, Englehart K. 2014. Motion Normalized Proportional Control for Improved Pattern Recognition-Based Myoelectric Control. IEEE Trans Neural Syst Rehabil Eng 22:149-157. doi:10.1109/TNSRE.2013.2247421

Schultz AE, Kuiken TA. 2011. Neural Interfaces for Control of Upper Limb Prostheses: The State of the Art and Future Possibilities. PM\&R 3:55-67. doi:10.1016/j.pmrj.2010.06.016

Scott RNN, Parker PAA. 1988. Myoelectric prostheses: state of the art. J Med Eng Technol 12:143-151.

Scovil CY, Ronsky JL. 2006. Sensitivity of a Hill-based muscle model to perturbations in model parameters. J Biomech 39:2055-2063. doi:10.1016/j.jbiomech.2005.06.005

Shadmehr R, Huang HJ, Ahmed AA. 2016. A Representation of Effort in Decision-Making and Motor Control. Curr Biol 26:1929-1934. doi:10.1016/j.cub.2016.05.065

Shaharudin S, Zanotto D, Agrawal S. 2014. Muscle Synergies of Untrained Subjects during 6 min Maximal Rowing on Slides and Fixed Ergometer. J Sports Sci Med 13:793-800.

Shik ML, Severin F V, Orlovsky GN. 1969. Control of walking and running by means of electrical stimulation of the mesencephalon. Electroencephalogr Clin Neurophysiol 26:549.

Shim JK, Oliveira MA, Hsu J, Huang J, Park J, Clark JE. 2007. Hand digit control in children: age-related changes in hand digit force interactions during maximum flexion and extension force production tasks. Exp Brain Res 176:374-386. doi:10.1007/s00221-006-0629-x

Sloot LH, van der Krogt MM, Harlaar J. 2014a. Effects of adding a virtual reality environment to different modes of treadmill walking. Gait Posture 39:939-945. doi:10.1016/j.gaitpost.2013.12.005

Sloot LH, van der Krogt MM, Harlaar J. 2014b. Self-paced versus fixed speed treadmill walking. Gait Posture 39:478-484. doi:10.1016/j.gaitpost.2013.08.022

Smith LH, Kuiken TA, Hargrove LJ. 2014. Real-time simultaneous and proportional myoelectric control using intramuscular EMG. J Neural Eng 11:066013. doi:10.1088/17412560/11/6/066013

Smith OJM. 1957. Posicast control of damped oscillatory systems. Proc IRE 1249-1255. doi:10.1109/JRPROC.1957.278530

Smutz WP, Kongsayreepong A, Hughes RE, Niebur G, Cooney WP, An KN. 1998. Mechanical advantage of the thumb muscles. J Biomech 31:565-70. doi:10.1016/s00219290(98)00043-8

Sobinov A, Boots MT, Gritsenko V, Fisher LE, Gaunt RA, Yakovenko S. 2019. Approximating complex musculoskeletal biomechanics using multidimensional autogenerating polynomials. bioRxiv. doi:10.1101/759878

Sobinov A, Yakovenko S. 2018. Model of a bilateral Brown-type central pattern generator for symmetric and asymmetric locomotion. J Neurophysiol 119:1071-1083. doi:10.1152/jn.00443.2017

Souman JL, Giordano PR, Schwaiger MC, Frissen I, Thümmel T, Ulbrich H, Luca A De, Bülthoff 109 
HH, Ernst MO. 2011. CyberWalk: Enabling Unconstrained Omnidirectional Walking through Virtual Environments. ACM Trans Appl Percept 8:1-22. doi:10.1145/2043603.2043607

Sousa CO, Barela JA, Prado-Medeiros CL, Salvini TF, Barela AMF. 2009. The use of body weight support on ground level: an alternative strategy for gait training of individuals with stroke. J Neuroeng Rehabil 6:43. doi:10.1186/1743-0003-6-43

Stavar A, Dascalu LM, Talaba D. 2011. Design, Test and Experimental Validation of a VR Treadmill Walking Compensation DevicelFIP Advances in Information and Communication Technology. pp. 402-409. doi:10.1007/978-3-642-19170-1_44

Stolze H, Kuhtz-Buschbeck J., Mondwurf C, Boczek-Funcke A, Jöhnk K, Deuschl G, Illert M. 1997. Gait analysis during treadmill and overground locomotion in children and adults. Electroencephalogr Clin Neurophysiol Mot Control 105:490-497. doi:10.1016/S0924980X(97)00055-6

Taylor DM. 2018. Americans With Disabilities: 2014 Household Economic Studies Current Population Reports.

Taylor MJD, Strike SC, Dabnichki P. 2006. Strategies Used for Unconstrained Direction Change during Walking. Percept Mot Skills 102:576-588. doi:10.2466/pms.102.2.576-588

Thelen DG. 2003. Adjustment of Muscle Mechanics Model Parameters to Simulate Dynamic Contractions in Older Adults. J Biomech Eng 125:70. doi:10.1115/1.1531112

Todorov E, Erez T, Tassa Y. 2012. MuJoCo: A physics engine for model-based control2012 IEEE/RSJ International Conference on Intelligent Robots and Systems. IEEE. pp. 50265033. doi:10.1109/IROS.2012.6386109

Torres-Oviedo G, Bastian AJ. 2010. Seeing Is Believing: Effects of Visual Contextual Cues on Learning and Transfer of Locomotor Adaptation. J Neurosci 30:17015-17022. doi:10.1523/JNEUROSCI.4205-10.2010

Torres-Oviedo G, Vasudevan E, Malone L, Bastian AJ. 2011. Locomotor adaptationProgress in Brain Research. pp. 65-74. doi:10.1016/B978-0-444-53752-2.00013-8

United Nations, Department of Economic and Social Affairs PD. 2015. World Population Ageing 2015.

van Soest AJ, Bobbert MF. 1993. The contribution of muscle properties in the control of explosive movements. Biol Cybern 69:195-204. doi:10.1007/BF00198959

Wagner FB, Mignardot J-B, Le Goff-Mignardot CG, Demesmaeker R, Komi S, Capogrosso M, Rowald A, Seáñez I, Caban M, Pirondini E, Vat M, McCracken LA, Heimgartner R, Fodor I, Watrin A, Seguin P, Paoles E, Van Den Keybus K, Eberle G, Schurch B, Pralong E, Becce F, Prior J, Buse N, Buschman R, Neufeld E, Kuster N, Carda S, von Zitzewitz J, Delattre V, Denison T, Lambert H, Minassian K, Bloch J, Courtine G. 2018. Targeted neurotechnology restores walking in humans with spinal cord injury. Nature 563:65-71. doi:10.1038/s41586018-0649-2

Weijs WA, Hillen B. 1985. Cross-sectional areas and estimated intrinsic strength of the human jaw muscles. Acta Morphol Neerl Scand 23:267-74.

Whiteside SR, Alaimo J, Barringer WJ, Beiswenger WD, Bulgarelli T, Hentges CJ, Lin RS, Miller TE, Parr RG, Reynolds JH, Stills ML, Greenberg S, Ellen A, Sawtell BA. 2000. Practice Analysis Task Force. Am BOARD Certif Orthot PROSTHETICS, INC. 
Williams TW. 1990. Practical Methods for Controlling Powered Upper-Extremity Prostheses. Assist Technol 2:3-18. doi:10.1080/10400435.1990.10132142

Winters JM, Stark L. 1987. Muscle models: What is gained and what is lost by varying model complexity. Biol Cybern 55:403-420. doi:10.1007/BF00318375

Yakovenko S. 2011. A hierarchical perspective on rhythm generation for locomotor controlProgress in Brain Research. Elsevier BV. pp. 151-166. doi:10.1016/B978-0-44453825-3.00015-2

Yakovenko S, Gritsenko V, Prochazka A. 2004. Contribution of stretch reflexes to locomotor control: a modeling study. Biol Cybern 90:146-155. doi:10.1007/s00422-003-0449-z

Yakovenko S, Krouchev N, Drew T. 2011. Sequential activation of motor cortical neurons contributes to intralimb coordination during reaching in the cat by modulating muscle synergies. J Neurophysiol 105:388-409. doi:10.1152/jn.00469.2010

Yakovenko S, Sobinov A, Gritsenko V. 2018. Analytical CPG model driven by limb velocity input generates accurate temporal locomotor dynamics. PeerJ 6:e5849. doi:10.7717/peerj.5849

Yoon J, Park H-S, Damiano D. 2012. A novel walking speed estimation scheme and its application to treadmill control for gait rehabilitation. J Neuroeng Rehabil 9:62. doi:10.1186/1743-0003-9-62

Zajac FE. 1989. Muscle and tendon: properties, models, scaling, and application to biomechanics and motor control. Crit Rev Biomed Eng 17:359-411.

Zhong G, Shevtsova NA, Rybak IA, Harris-Warrick RM. 2012. Neuronal activity in the isolated mouse spinal cord during spontaneous deletions in fictive locomotion: insights into locomotor central pattern generator organization. J Physiol 590:4735-4759. doi:10.1113/jphysiol.2012.240895

Ziegler-Graham K, MacKenzie EJ, Ephraim PL, Travison TG, Brookmeyer R. 2008. Estimating the Prevalence of Limb Loss in the United States: 2005 to 2050. Arch Phys Med Rehabil 89:422-429. doi:10.1016/j.apmr.2007.11.005 